\title{
COMBINATORICS OF BI-FREENESS WITH AMALGAMATION
}

\author{
IAN CHARLESWORTH, BRENT NELSON, AND PAUL SKOUFRANIS
}

\begin{abstract}
In this paper, we develop the theory of bi-freeness in an amalgamated setting. We construct the operator-valued bi-free cumulant functions, and show that the vanishing of mixed cumulants is necessary and sufficient for bi-free independence. Further, we develop a multiplicative convolution for operator-valued random variables and explore ways to construct bi-free pairs of $B$-faces.
\end{abstract}

\section{INTRODUCTION}

In [7, Voiculescu introduced the notion of bi-free probability as a generalization of free probability to study of left and right actions of algebras on a reduced free product space simultaneously. Voiculescu demonstrated that many results from free probability have direct analogues in the bi-free setting. In particular, 7. demonstrated the existence of bi-free cumulant polynomials an analogue to free cumulants, although it did not produce an explicit formula.

Mastnak and Nica defined the $(\ell, r)$-cumulant functions in [2, via permutations applied to non-crossing partitions. In addition, they hypothesized that the $(\ell, r)$-cumulants were the correct cumulant functions for bi-free probability and gave rise to the bi-free cumulant polynomials of Voiculescu. In [1, the authors of this paper proved this was the case via a diagrammatic argument. In addition, we constructed an intuitive multiplicative convolution and a complicated operator model on a Fock space for a pair of faces.

In [7. Section 8], Voiculescu laid the framework for generalizing bi-free probability to an amalgamated setting. As the combinatorics of free probability with amalgamation are well-understood (c.f. [3 [5]), the goal of this paper is to demonstrate that the results of [1] generalize to an amalgamated setting and that the combinatorics of bi-freeness with amalgamation are natural extensions of the combinatorics of free probability with amalgamation.

This paper contains ten sections beyond this introduction, structured as follows. Section 2 recalls the necessary background from 1. In particular, the notion of bi-non-crossing partitions, their lateral refinements, the incident algebra for bi-non-crossing partitions, and the structure of the universal polynomials for moments of bi-free pairs of faces will be reviewed.

Section 3 introduces the setting for bi-free probability with amalgamation from [7. We define the notion of a $B$-B-non-commutative probability space $(\mathcal{A}, E, \varepsilon)$ (Definition 3.2.1) and demonstrate a representation of $\mathcal{A}$ as linear operators on a $B$-B-bimodule (see Theorem 3.2.4). In addition, the notion of bi-free pairs of $B$-faces is reintroduced (see Definition 3.3.1).

Section 4 introduces the notion of an operator-valued bi-multiplicative function (see Definition 4.2.1). Such functions are extensions of multiplicative functions (see [3, Section 2] or [5, Section 2]) to the bi-free setting but have natural descriptions via multiplicative functions (see Remark 4.2.4).

Section 5 defines certain terms $E_{\pi}\left(T_{1}, \ldots, T_{n}\right)$ (see Definition 5.1.1) which appear when actions of pairs of $B$-faces are examined and give rise to an operator-valued bi-multiplicative moment function. Unfortunately, Section 5 is fairly technical due to the lack of an analogue of centring techniques from free-probability.

Section [6]defines the operator-valued bi-free cumulants (see Definition 6.1.3) as a convolution of the Möbius function for bi-non-crossing partitions with $E_{\pi}\left(T_{1}, \ldots, T_{n}\right)$ and demonstrates that they are bi-multiplicative (see Corollary 6.2.2). In addition, we show that the operator-valued bi-free cumulants posess a certain property analogous to the property of operator-valued free cumulants demonstrated in [5, Section 3.2] (see Section 6.3) and vanish when a left or right $B$-operator is input (see Proposition 6.4.1).

Section 7 demonstrates through Theorem 7.1 .4 that a family of pairs of $B$-faces are bi-free with amalgamation over $B$ if and only if certain universal moment polynomials involving $E_{\pi}\left(T_{1}, \ldots, T_{n}\right)$ are satisfied.

This research was supported in part by NSF grants DMS-1161411, DMS-0838680 and by NSERC PGS-6799-438440-2013. 
The main result of this paper, Theorem 8.1.1 then follows immediately in Section 8 a family of pairs of $B$-faces are bi-free over $B$ if and only if their mixed operator-valued bi-free cumulants vanish.

Section 9 demonstrates how operator-valued bi-free cumulants involving products of operators may be computed. Also, Proposition 9.2.1 (which holds only in the scalar setting) generalizes [1, Theorem 5.2.1] from the multiplicative convolution for bi-free two-faced families with singletons in each left and each right face to an arbitrary number of operators in each face.

Finally, Section 10 provides additional methods for constructing bi-free pairs of $B$-faces by showing that conjugating a pair of $B$-faces by a $B$-valued Haar bi-unitary produces a pair of $B$-faces bi-free from the original pair, and that the bi-freeness of pairs of $B$-faces where all left faces commute with all right faces is intrinsically related to the freeness of just the left or just the right faces.

\section{Background on Bi-Non-Crossing Partitions}

We begin by reviewing many of the results from [1], which we will extend to the amalgamated setting. Throughout this section, let $n \in \mathbb{N}, \chi:\{1, \ldots, n\} \rightarrow\{\ell, r\}$, and $\epsilon:\{1, \ldots, n\} \rightarrow K$ for some fixed set $K$.

2.1. Bi-Non-Crossing Partitions and Diagrams. Recall that $\chi$ induces a permutation $s_{\chi} \in S_{n}$ corresponding to reading the left nodes in increasing order, followed by the right nodes in decreasing order: if $\chi^{-1}(\{\ell\})=\left\{i_{1}<\cdots<i_{p}\right\}$ and $\chi^{-1}(\{r\})=\left\{i_{p+1}>\cdots>i_{n}\right\}$, we set $s_{\chi}(k)=i_{k}$. Recall also that the set of partitions on $n$ elements, $\mathcal{P}(n)$, is partially ordered by refinement: for $\pi, \sigma \in \mathcal{P}(n)$, we have $\pi \leq \sigma$ if and only if every block of $\pi$ is contained in a single block of $\sigma$. Finally, let $|\sigma|$ denote the number of blocks in $\sigma$.

Definition 2.1.1. A partition $\pi \in \mathcal{P}(n)$ is said to be bi-non-crossing with respect to $\chi$ if the partition $s_{\chi}^{-1} \cdot \pi \in N C(n)$ - that is, the partition formed by applying $s_{\chi}^{-1}$ to the blocks of $\pi$ - is non-crossing. Equivalently, $\pi$ is bi-non-crossing if whenever there are blocks $U, V \in \pi$ with $u_{1}, u_{2} \in U$ and $v_{1}, v_{2} \in V$ such that $s_{\chi}^{-1}\left(u_{1}\right)<s_{\chi}^{-1}\left(v_{1}\right)<s_{\chi}^{-1}\left(u_{2}\right)<s_{\chi}^{-1}\left(v_{2}\right)$, we have $U=V$. The set of bi-non-crossing partitions with respect to $\chi$ is denoted $B N C(\chi)$, and inherits a lattice structure from $\mathcal{P}(n)$. We will use $0_{\chi}$ and $1_{\chi}$ to denote the minimal and maximal elements of $B N C(\chi)$, respectively.

To each partition $\pi \in B N C(\chi)$ we can associate a "bi-non-crossing diagram" by placing nodes along two vertical lines, labelled 1 to $n$ from top to bottom, such that the nodes on the left line correspond to those values for which $\chi(k)=\ell$ (similarly for the right), and connecting those nodes which are in the same block of $\pi$ in a non-crossing manner.

Example 2.1.2. If $\chi^{-1}(\{l\})=\{1,2,4\}, \chi^{-1}(\{r\})=\{3,5\}$, and

$$
\pi=\{\{1,3\},\{2,4,5\}\}=s_{\chi} \cdot\{\{1,5\},\{2,3,4\}\},
$$

then the bi-non-crossing diagram associated to $\pi$ is

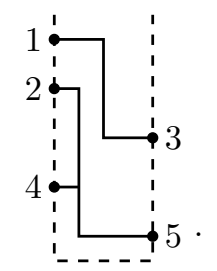

Definition 2.1.3. The set $L R(\chi, \epsilon)$ of shaded $L R$ diagrams is defined recursively. If $n=0, L R(\chi, \epsilon)$ consists of an empty diagram. If $n>0$, let $\bar{\chi}(k)=\chi(k-1)$ for $k \in\{2, \ldots, n\}$ and $\bar{\epsilon}(k)=\epsilon(k-1)$ for $k \in\{2, \ldots, n\}$. Each $D \in L R(\bar{\chi}, \bar{\epsilon})$ then corresponds to two (unique) elements of $L R(\chi, \epsilon)$ via the following process:

- First, add to the top of $D$ a node on the side corresponding to $\chi(1)$, shaded by $\epsilon(1)$.

- If a string of shade $\epsilon(1)$ extends from the top of $D$, connect it to the added node.

- Then, choose to either extend a string from the added node to the top of the new diagram or not, and extend any other strings from $D$ to the top of the new diagram.

We will denote the subset of $L R(\chi, \epsilon)$ consisting of diagrams with exactly $k$ strings that reach the top by $L R_{k}(\chi, \epsilon)$.

We refer to the vertical portions of strings in $L R$ and bi-non-crossing diagrams as spines, and horizontal segments connecting nodes to spines as ribs. 
Note that any element of $L R_{0}(\chi, \epsilon)$ corresponds to a partition $\pi \in B N C(\chi)$ by taking as blocks in $\pi$ each set of nodes joined by strings in the diagram. The non-crossing diagram corresponding to $\pi$ will then match the original $L R$-diagram with the shades removed.

Example 2.1.4. Consider $\chi=(\ell, r)$ and $\epsilon=\left({ }^{\prime},{ }^{\prime \prime}\right)$. Then $L R(\chi, \epsilon)$ consists of the following diagrams:
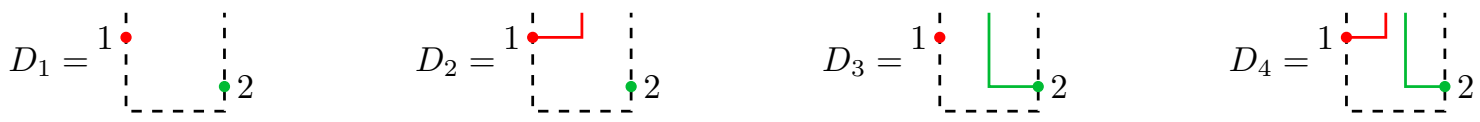

Here $L R_{0}(\chi, \epsilon)=\left\{D_{1}\right\}$.

Example 2.1.5. For a slightly more robust example we consider $\chi=(r, \ell, r)$ and $\epsilon=\left({ }^{\prime},{ }^{\prime \prime},{ }^{\prime}\right)$. Then $L R(\chi, \epsilon)$ consists of the following diagrams:
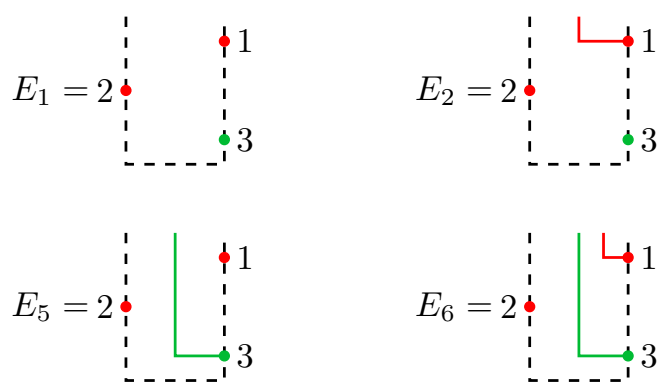

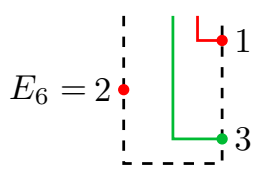

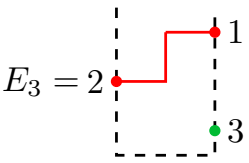

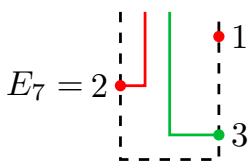

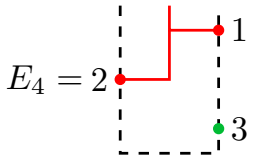

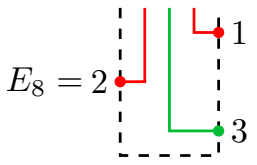

In this case, $L R_{0}(\chi, \epsilon)=\left\{E_{1}, E_{3}\right\}$. We also note that $D_{k}$ from Example 2.1.4 gives rise to diagrams $E_{2 k-1}$ and $E_{2 k}$.

These shaded LR diagrams are useful because the choices made when constructing them correspond to the choices made in expanding a mixed moment of bi-free random variables in terms of pure moments. In doing so, we will often consider a map $\alpha:\{1, \ldots, n\} \rightarrow I \sqcup J$ where $I$ and $J$ are index sets of left and right random variables, respectively. We will want to consider the bi-non-crossing diagrams corresponding to such a situation, and so, as useful shorthand, we will define $\chi_{\alpha}:\{1, \ldots, n\} \rightarrow\{\ell, r\}$ by $\chi_{\alpha}^{-1}(\{\ell\})=\alpha^{-1}(I)$, and take $B N C(\alpha)=B N C\left(\chi_{\alpha}\right)$.

2.2. Combinatorics on Bi-Non-Crossing Partitions. Recall that the incident algebra $I A(B N C)$ on the set of bi-non-crossing partitions is the algebra of functions of the form

$$
f: \bigcup_{n \geq 1} \bigcup_{\chi:\{1, \ldots, n\} \rightarrow\{\ell, r\}} B N C(\chi) \times B N C(\chi) \longrightarrow \mathbb{C}
$$

such that $f(\pi, \sigma)=0$ unless $\pi$ is a refinement of $\sigma$, with point-wise addition, and multiplication given by convolution: if $f, g \in I A(B N C)$ and $\pi, \sigma \in B N C(\chi)$,

$$
(f * g)(\pi, \sigma)=\sum_{\pi \leq \rho \leq \sigma} f(\pi, \rho) g(\rho, \sigma) .
$$

As was shown in [1, we can associate to each interval in $B N C(\chi)$ a product of full intervals. We say a function $f \in I A(B N C)$ is multiplicative if whenever $[\pi, \sigma]$ corresponds to $\prod_{j=1}^{k} B N C\left(\beta_{k}\right)$, we have

$$
f(\pi, \sigma)=\prod_{j=1}^{k} f\left(0_{\beta_{k}}, 1_{\beta_{k}}\right) .
$$

We note that a multiplicative function $f$ is completely determined by the values it takes on each $\left(0_{\chi}, 1_{\chi}\right)$.

The multiplicative identity in $I A(B N C)$ is given by the delta function

$$
\delta_{B N C}(\pi, \sigma)=\left\{\begin{array}{ll}
1 & \text { if } \pi=\sigma \\
0 & \text { otherwise }
\end{array} .\right.
$$

We then define the zeta function on $I A(B N C)$ by

$$
\zeta_{B N C}(\pi, \sigma)=\left\{\begin{array}{ll}
1 & \text { if } \pi \leq \sigma \\
0 & \text { otherwise }
\end{array},\right.
$$


and the Möbius function $\mu_{B N C}$ as its multiplicative inverse:

$$
\mu_{B N C} * \zeta_{B N C}=\delta_{B N C}=\zeta_{B N C} * \mu_{B N C} .
$$

It can be shown that these three functions are multiplicative and $\mu_{B N C}(\pi, \sigma)=\mu_{N C}\left(s_{\chi}^{-1} \cdot \pi, s_{\chi}^{-1} \cdot \sigma\right)$.

Suppose that $T_{1}, \ldots, T_{n}$ are elements of a non-commutative probability space $(\mathcal{A}, \varphi)$ and $\pi \in B N C(\chi)$ with blocks $V_{t}=\left\{k_{t, 1}<\cdots<k_{t, m_{t}}\right\}, t \in\{1, \ldots, j\}$. We set the notation

$$
\varphi_{\pi}\left(T_{1}, \ldots, T_{n}\right):=\prod_{t=1}^{j} \varphi\left(T_{k_{t, 1}} \cdots T_{k_{t, m_{t}}}\right)
$$

and

$$
\kappa_{\pi}\left(T_{1}, \ldots, T_{n}\right):=\sum_{\substack{\sigma \in B N C(\chi) \\ \sigma \leq \pi}} \varphi_{\sigma}\left(T_{1}, \ldots, T_{n}\right) \mu_{B N C}(\sigma, \pi) .
$$

It was shown in [1] that $\kappa_{1_{\chi}}\left(T_{1}, \ldots, T_{n}\right)$ are the $(\ell, r)$-cumulants as defined in [2, Definition 5.2].

2.3. Bi-free Probability. We now recall several definitions relating to bi-free probability from [7].

Definition 2.3.1. Let $(\mathcal{A}, \varphi)$ be a non-commutative probability space: that is, let $\mathcal{A}$ be a unital algebra and $\varphi: \mathcal{A} \rightarrow \mathbb{C}$ be unital and linear. A pair of faces in $\mathcal{A}$ is a pair $(C, D)$ of unital subalgebras of $\mathcal{A}$. We will call $C$ the left face and $D$ the right face.

Definition 2.3.2. A family $\left\{\left(C_{k}, D_{k}\right)\right\}_{k \in K}$ of pairs of faces in a non-commutative probability space $(\mathcal{A}, \varphi)$ is said to be bi-freely independent if there exists a family of vector spaces with specified vector states $\left\{\left(\mathcal{X}_{k}, \stackrel{\circ}{\mathcal{X}}_{k}, \xi_{k}\right)\right\}_{k \in K}$ and unital homomorphisms

$$
l_{k}: C_{k} \rightarrow \mathcal{L}\left(\mathcal{X}_{k}\right) \quad \text { and } \quad r_{k}: D_{k} \rightarrow \mathcal{L}\left(\mathcal{X}_{k}\right)
$$

such that the joint distribution of the family with respect to $\varphi$ is equal to the joint distribution with respect to the vacuum state on the representation on $*_{k \in K}\left(\mathcal{X}_{k}, \dot{\mathcal{X}}_{k}, \xi_{k}\right)$.

Remark 2.3.3. It is sometimes useful to think of $\epsilon:\{1, \ldots, n\} \rightarrow K$ not as a map but rather as the partition $\epsilon^{-1}(K)$ it induces. Thus if we write $\sigma \leq \epsilon$ for some partition $\sigma$, we mean that $\sigma$ is a refinement of $\epsilon^{-1}(K)$ and so $\epsilon$ is constant on each block in $\sigma$.

Definition 2.3.4. Suppose $\pi, \sigma \in B N C(\chi)$ are such that $\pi \leq \sigma$. We say $\pi$ is a lateral refinement of $\sigma$ and write $\pi \leq_{\text {lat }} \sigma$ if the bi-non-crossing diagram for $\pi$ can be obtained from that of $\sigma$ by making lateral "cuts" along the spines of blocks of $\pi$ between their ribs; that is, by removing some portion of the vertical lines and then any horizontal lines that are no longer attached to a vertical line in the diagram from $\sigma$.

In [1, Proposition 4.2.1], we established the following combinatorial result which was crucial in connecting bi-freeness with cumulants. It will once again prove useful in this paper.

Theorem 2.3.5. Let $\chi:\{1, \ldots, n\} \rightarrow\{\ell, r\}$ and $\epsilon:\{1, \ldots, n\} \rightarrow K$. Then for every $\pi \in B N C(\chi)$ such that $\pi \leq \epsilon$,

$$
\sum_{\substack{\sigma \in L R_{0}(\chi, \epsilon) \\ \sigma \geq_{\text {lat }} \pi}}(-1)^{|\pi|-|\sigma|}=\sum_{\substack{\sigma \in B N C(\chi) \\ \pi \leq \sigma \leq \epsilon}} \mu_{B N C}(\pi, \sigma) .
$$

We desire to extend the following result, [1, Theorem 4.3.1], to the amalgamated setting.

Theorem 2.3.6. Let $(\mathcal{A}, \varphi)$ be a non-commutative probability space and let $\left\{\left(C_{k}, D_{k}\right)\right\}_{k \in K}$ be a family of pairs of faces from $\mathcal{A}$. Then $\left\{\left(C_{k}, D_{k}\right)\right\}_{k \in K}$ are bi-free if and only if for all $\chi:\{1, \ldots, n\} \rightarrow\{\ell, r\}$, $\epsilon:\{1, \ldots, n\} \rightarrow K$ non-constant, and

$$
T_{k} \in\left\{\begin{array}{ll}
C_{\epsilon(k)} & \text { if } \chi(k)=\ell \\
D_{\epsilon(k)} & \text { if } \chi(k)=r
\end{array},\right.
$$

we have

$$
\kappa_{1_{\chi}}\left(T_{1}, \ldots, T_{n}\right)=0
$$


Equivalently, for all $\chi:\{1, \ldots, n\} \rightarrow\{\ell, r\}$, for all $\epsilon:\{1, \ldots, n\} \rightarrow K$, and for all $T_{k}$ as defined above,

$$
\varphi\left(T_{1} \cdots T_{n}\right)=\sum_{\pi \in B N C(\chi)}\left[\sum_{\substack{\sigma \in B N C(\chi) \\ \pi \leq \sigma \leq \epsilon}} \mu_{B N C}(\pi, \sigma)\right] \varphi_{\pi}\left(T_{1}, \ldots, T_{n}\right) .
$$

\section{Bi-Free Families with Amalgamation}

In this section, we will recall and develop the structures from [7, Section 8] necessary to discuss bi-freeness with amalgamation. Throughout the paper, $B$ will denote a unital algebra over $\mathbb{C}$.

3.1. Concrete Structures for Bi-Free Probability with Amalgamation. To begin the necessary constructions in the amalgamated setting, we need an analogue of a vector space with a specified vector state.

Definition 3.1.1. A $B$-B-bimodule with a specified $B$-vector state is a triple $(\mathcal{X}, \mathcal{X}, p)$ where $\mathcal{X}$ is a direct sum of $B$ - $B$-bimodules

$$
\mathcal{X}=B \oplus \dot{\mathcal{X}}
$$

and $p: \mathcal{X} \rightarrow B$ is the linear map

$$
p(b \oplus \eta)=b .
$$

Remark 3.1.2. Given a $B$ - $B$-bimodule with a specified $B$-vector state $(\mathcal{X}, \mathcal{X}, p)$, for $b_{1}, b_{2} \in B$ and $\eta \in \mathcal{X}$ we have

$$
p\left(b_{1} \cdot \eta \cdot b_{2}\right)=b_{1} p(\eta) b_{2} .
$$

Definition 3.1.3. Given a $B$ - $B$-bimodule with a specified $B$-vector state $(\mathcal{X}, \mathcal{X}, p)$, let $\mathcal{L}(\mathcal{X})$ denote the set of linear operators on $\mathcal{X}$. Given $b \in B$, we define two operators $L_{b}, R_{b} \in \mathcal{L}(\mathcal{X})$ by

$$
L_{b}(\eta)=b \cdot \eta \quad \text { and } \quad R_{b}(\eta)=\eta \cdot b \quad \text { for } \eta \in \mathcal{X} .
$$

In addition, we define the unital subalgebras $\mathcal{L}_{\ell}(\mathcal{X})$ and $\mathcal{L}_{r}(\mathcal{X})$ of $\mathcal{L}(\mathcal{X})$ by

$$
\begin{aligned}
& \mathcal{L}_{\ell}(\mathcal{X}):=\left\{T \in \mathcal{L}(\mathcal{X}) \mid T R_{b}=R_{b} T \text { for all } b \in B\right\} \\
& \mathcal{L}_{r}(\mathcal{X}):=\left\{T \in \mathcal{L}(\mathcal{X}) \mid T L_{b}=L_{b} T \text { for all } b \in B\right\}
\end{aligned}
$$

We call $\mathcal{L}_{\ell}(\mathcal{X})$ and $\mathcal{L}_{r}(\mathcal{X})$ the left and right algebras of $\mathcal{L}(\mathcal{X})$, respectively.

Remark 3.1.4. Note $\mathcal{L}_{\ell}(\mathcal{X})$ consists of all operators in $\mathcal{L}(\mathcal{X})$ that are right $B$-linear; that is, if $T \in \mathcal{L}_{\ell}(\mathcal{X})$ then

$$
T(\eta \cdot b)=T\left(R_{b}(\eta)\right)=R_{b}(T(\eta))=T(\eta) \cdot b
$$

for all $b \in B$ and $\eta \in \mathcal{X}$. In the usual treatment of bimodules, what we have denoted $\mathcal{L}_{\ell}(\mathcal{X})$ would instead be $\mathcal{L}_{r}(\mathcal{X})$ and vice versa, to reflect the fact that its elements are right $B$-linear. However, we take our left (resp. right) face to be a sub-algebra of $\mathcal{L}_{\ell}(\mathcal{X})\left(\right.$ resp. $\mathcal{L}_{r}(\mathcal{X})$ ). Moreover, one sees from the $B$ - $B$-bimodule structure that $b \mapsto L_{b}$ is a homomorphism, $b \mapsto R_{b}$ is an anti-homomorphism, and the ranges of these maps commute. Hence

$$
\left\{L_{b} \mid b \in B\right\} \subseteq \mathcal{L}_{\ell}(\mathcal{X}) \quad \text { and } \quad\left\{R_{b} \mid b \in B\right\} \subseteq \mathcal{L}_{r}(\mathcal{X})
$$

Thus, in the context of this paper, $\mathcal{L}_{\ell}(\mathcal{X})$ consists of 'left' operators and $\mathcal{L}_{r}(\mathcal{X})$ consists of 'right' operators.

As we are interested in $\mathcal{L}(\mathcal{X})$ and amalgamating over $B$, we will need an "expectation" from $\mathcal{L}(\mathcal{X})$ to $B$.

Definition 3.1.5. Given a $B$ - $B$-bimodule with a specified $B$-vector state $(\mathcal{X}, \mathcal{X}, p)$, we define the linear $\operatorname{map} E_{\mathcal{L}(\mathcal{X})}: \mathcal{L}(\mathcal{X}) \rightarrow B$ by

$$
E_{\mathcal{L}(\mathcal{X})}(T)=p\left(T\left(1_{B} \oplus 0\right)\right)
$$

for all $T \in \mathcal{L}(\mathcal{X})$. We call $E_{\mathcal{L}(\mathcal{X})}$ the expectation of $\mathcal{L}(\mathcal{X})$ onto $B$.

The following important properties justify calling $E_{\mathcal{L}(\mathcal{X})}$ an expectation. 
Proposition 3.1.6. Let $(\mathcal{X}, \mathcal{X}, p)$ be a $B$-B-bimodule with a specified $B$-vector state. Then

$$
E_{\mathcal{L}(\mathcal{X})}\left(L_{b_{1}} R_{b_{2}} T\right)=b_{1} E_{\mathcal{L}(\mathcal{X})}(T) b_{2}
$$

for all $b_{1}, b_{2} \in B$ and $T \in \mathcal{L}(\mathcal{X})$, and

$$
E_{\mathcal{L}(\mathcal{X})}\left(T L_{b}\right)=E_{\mathcal{L}(\mathcal{X})}\left(T R_{b}\right)
$$

for all $b \in B$ and $T \in \mathcal{L}(\mathcal{X})$.

Proof. If $b_{1}, b_{2} \in B$ and $T \in \mathcal{L}(\mathcal{X})$, we see that

$$
E_{\mathcal{L}(\mathcal{X})}\left(L_{b_{1}} R_{b_{2}} T\right)=p\left(L_{b_{1}} R_{b_{2}} T\left(1_{B} \oplus 0\right)\right)=p\left(L_{b_{1}} R_{b_{2}}(E(T) \oplus \eta)\right)=p\left(\left(b_{1} E(T) b_{2}\right) \oplus\left(b_{1} \cdot \eta \cdot b_{2}\right)\right)=b_{1} E(T) b_{2}
$$

where $\eta \in \dot{\mathcal{X}}$. The second result is immediate from $L_{b}\left(1_{B} \oplus 0\right)=R_{b}\left(1_{B} \oplus 0\right)$.

To complete this section, we recall the construction of the reduced free product of $B$ - $B$-bimodules with specified $B$-vector states.

Construction 3.1.7. Let $\left\{\left(\mathcal{X}_{k}, \stackrel{\circ}{\mathcal{X}}_{k}, p_{k}\right)\right\}_{k \in K}$ be $B$-B-bimodules with specified $B$-vector states. For simplicity, let $E_{k}$ denote $E_{\mathcal{L}\left(\mathcal{X}_{k}\right)}$. The free product of $\left\{\left(\mathcal{X}_{k}, \dot{\mathcal{X}}_{k}, p_{k}\right)\right\}_{k \in K}$ with amalgamation over $B$ is defined to be the $B$ - $B$-bimodule with specified vector state $(\mathcal{X}, \dot{\mathcal{X}}, p)$ where $\mathcal{X}=B \oplus \stackrel{\mathcal{X}}{ }$ and $\stackrel{\mathcal{X}}{\text { is }}$ the $B$ - $B$-bimodule

$$
\dot{\mathcal{X}}=\bigoplus_{n \geq 1} \bigoplus_{k_{1} \neq k_{2} \neq \cdots \neq k_{n}} \dot{\mathcal{X}}_{k_{1}} \otimes_{B} \cdots \otimes_{B} \dot{\mathcal{X}}_{k_{n}}
$$

with the left and right actions of $B$ on $\dot{\mathcal{X}}$ defined by

$$
\begin{aligned}
& b \cdot\left(x_{1} \otimes \cdots \otimes x_{n}\right)=\left(L_{b} x_{1}\right) \otimes \cdots \otimes x_{n} \\
& \left(x_{1} \otimes \cdots \otimes x_{n}\right) \cdot b=x_{1} \otimes \cdots \otimes\left(R_{b} x_{n}\right) .
\end{aligned}
$$

We use $*_{k \in K} \mathcal{X}_{k}$ to denote $\mathcal{X}$.

For each $k \in K$, we define the left representation $\lambda_{k}: \mathcal{L}\left(\mathcal{X}_{k}\right) \rightarrow \mathcal{L}(\mathcal{X})$ as follows: let

$$
V_{k}: \mathcal{X} \rightarrow \mathcal{X}_{k} \otimes_{B}\left(B \oplus \bigoplus_{n \geq 1} \bigoplus_{\substack{k_{1} \neq k_{2} \neq \cdots \neq k_{n} \\ k_{1} \neq k}} \dot{\mathcal{X}}_{k_{1}} \otimes_{B} \cdots \otimes_{B} \dot{\mathcal{X}}_{k_{n}}\right)
$$

be the standard $B$ - $B$-bimodule isomorphism and set

$$
\lambda_{k}(T)=V_{k}^{-1}(T \otimes I) V_{k} .
$$

More precisely,

$$
\lambda_{k}(T)(b)=E_{k}(T) b \oplus\left(T-L_{E_{k}(T)}\right) b,
$$

and

$$
\lambda_{k}(T)\left(x_{1} \otimes \cdots \otimes x_{n}\right)=\left\{\begin{array}{ll}
\left(L_{p_{k}\left(T x_{1}\right)} x_{2} \otimes \cdots \otimes x_{n}\right) \oplus\left(\left[\left(1-p_{k}\right) T x_{1}\right] \otimes \cdots \otimes x_{n}\right) & \text { if } x_{1} \in \dot{\mathcal{X}}_{k} \\
\left(L_{E_{k}(T)} x_{1} \otimes \cdots \otimes x_{n}\right) \oplus\left(\left[\left(T-L_{E_{k}(T)}\right) 1_{B}\right] \otimes x_{1} \otimes \cdots \otimes x_{n}\right) & \text { if } x_{1} \notin \dot{\mathcal{X}}_{k}
\end{array},\right.
$$

for all $T \in \mathcal{L}\left(\mathcal{X}_{k}\right)$ (where the tensor of length zero is $1_{B}$ ). Observe that $\lambda_{k}$ is a homomorphism, $\lambda_{k}\left(L_{b}\right)=L_{b}$, and $\lambda_{k}\left(\mathcal{L}_{\ell}\left(\mathcal{X}_{k}\right)\right) \subseteq \mathcal{L}_{\ell}(\mathcal{X})$

Similarly, for each $k \in K$, we define the map $\rho_{k}: \mathcal{L}\left(\mathcal{X}_{k}\right) \rightarrow \mathcal{L}(\mathcal{X})$ as follows: let

$$
W_{k}: \mathcal{X} \rightarrow\left(B \oplus \bigoplus_{\substack{n \geq 1 \\ k_{1} \neq k_{2} \neq \cdots \neq k_{n} \\ k_{n} \neq k}} \dot{\mathcal{X}}_{k_{1}} \otimes_{B} \cdots \otimes_{B} \dot{\mathcal{X}}_{k_{n}}\right) \otimes_{B} \mathcal{X}_{k}
$$

be the standard $B$ - $B$-bimodule isomorphism and define

$$
\rho_{k}(T)=W_{k}^{-1}(I \otimes T) W_{k} .
$$

Again,

$$
\rho_{k}(T)(b)=b E_{k}(T) \oplus\left(T-R_{E_{k}(T)}\right) b,
$$


and

$$
\rho_{k}(T)\left(x_{1} \otimes \cdots \otimes x_{n}\right)=\left\{\begin{array}{cc}
\left(x_{1} \otimes \cdots \otimes R_{p_{k}\left(T x_{n}\right)} x_{n-1}\right) \oplus\left(x_{1} \otimes \cdots \otimes\left[\left(1-p_{k}\right) T x_{n}\right]\right) & \text { if } x_{n} \in \dot{\mathcal{X}}_{k} \\
\left(x_{1} \otimes \cdots \otimes R_{E_{k}(T)} x_{n}\right) \oplus\left(x_{1} \otimes \cdots \otimes x_{n} \otimes\left[\left(T-R_{E_{k}(T)}\right) 1_{B}\right]\right) & \text { if } x_{n} \notin \dot{\mathcal{X}}_{k}
\end{array},\right.
$$

for all $T \in \mathcal{L}\left(\mathcal{X}_{k}\right)$. Clearly $\rho_{k}$ is an anti-homomorphism, $\rho_{k}\left(R_{b}\right)=R_{b}$, and $\rho_{k}\left(\mathcal{L}_{r}\left(\mathcal{X}_{k}\right)\right) \subseteq \mathcal{L}_{r}(\mathcal{X})$.

In addition, note if $T \in \mathcal{L}\left(\mathcal{X}_{k}\right)$ then

$$
E_{\mathcal{L}(\mathcal{X})}\left(\lambda_{k}(T)\right)=p\left(\lambda_{k}(T) 1_{B}\right)=p\left(E_{k}(T) \oplus\left[T-L_{E_{k}(T)}\right] 1_{B}\right)=E_{k}(T)
$$

and similarly $E_{\mathcal{L}(\mathcal{X})}\left(\rho_{k}(T)\right)=E_{k}(T)$. Hence, the above shows that $\mathcal{L}(\mathcal{X})$ contains each $\mathcal{L}\left(\mathcal{X}_{k}\right)$ in a leftpreserving, right-preserving, state-preserving way.

Remark 3.1.8. With computation, we see that $\lambda_{i}(T)$ and $\rho_{j}(S)$ commute when $T \in \mathcal{L}_{\ell}\left(\mathcal{X}_{i}\right), S \in \mathcal{L}_{r}\left(\mathcal{X}_{j}\right)$, and $i \neq j$. Indeed, notice if $b \in B$ then

$$
\begin{aligned}
& \lambda_{i}(T) \rho_{j}(S) b \\
& =\lambda_{i}(T)\left(b E_{j}(S) \oplus\left(S-R_{E_{j}(S)}\right) b\right) \\
& =E_{i}(T) b E_{j}(S) \oplus\left(T-L_{E_{i}(T)}\right) b E_{j}(S) \oplus L_{E_{i}(T)}\left(S-R_{E_{j}(S)}\right) b \oplus\left(\left[\left(T-L_{E_{i}(T)}\right) 1_{B}\right] \otimes\left[\left(S-R_{E_{j}(S)}\right) b\right]\right),
\end{aligned}
$$

whereas

$$
\begin{aligned}
& \rho_{j}(S) \lambda_{i}(T) b \\
& =\rho_{j}(S)\left(E_{i}(T) b \oplus\left(T-L_{E_{i}(T)}\right) b\right) \\
& =E_{i}(T) b E_{j}(S) \oplus\left(S-R_{E_{j}(S)}\right) E_{i}(T) b \oplus R_{E_{j}(S)}\left(T-L_{E_{i}(T)}\right) b \oplus\left(\left[\left(T-L_{E_{i}(T)}\right) b\right] \otimes\left[\left(S-R_{E_{j}(S)}\right) 1_{B}\right]\right) .
\end{aligned}
$$

Since $T \in \mathcal{L}_{\ell}\left(\mathcal{X}_{i}\right)$ and $S \in \mathcal{L}_{r}\left(\mathcal{X}_{j}\right)$, one sees that

$$
\begin{aligned}
& L_{E_{i}(T)}\left(S-R_{E_{j}(S)}\right) b=\left(S-R_{E_{j}(S)}\right) L_{E_{i}(T)} b=\left(S-R_{E_{j}(S)}\right) E_{i}(T) b, \\
& R_{E_{j}(S)}\left(T-L_{E_{i}(T)}\right) b=\left(T-L_{E_{i}(T)}\right) R_{E_{j}(S)} b=\left(T-L_{E_{i}(T)}\right) b E_{j}(S),
\end{aligned}
$$

and

$$
\begin{aligned}
{\left[\left(T-L_{E_{i}(T)}\right) b\right] \otimes\left[\left(S-R_{E_{j}(S)}\right) 1_{B}\right] } & =\left[\left(T-L_{E_{i}(T)}\right) R_{b} 1_{B}\right] \otimes\left[\left(S-R_{E_{j}(S)}\right) 1_{B}\right] \\
& =\left[R_{b}\left(T-L_{E_{i}(T)}\right) 1_{B}\right] \otimes\left[\left(S-R_{E_{j}(S)}\right) 1_{B}\right] \\
& =\left[\left(T-L_{E_{i}(T)}\right) 1_{B}\right] \otimes\left[L_{b}\left(S-R_{E_{j}(S)}\right) 1_{B}\right] \\
& =\left[\left(T-L_{E_{i}(T)}\right) 1_{B}\right] \otimes\left[\left(S-R_{E_{j}(S)}\right) L_{b} 1_{B}\right] \\
& =\left[\left(T-L_{E_{i}(T)}\right) 1_{B}\right] \otimes\left[\left(S-R_{E_{j}(S)}\right) b\right] .
\end{aligned}
$$

Thus $\lambda_{i}(T) \rho_{j}(S) b=\rho_{j}(S) \lambda_{i}(T) b$. Similar computations show $\lambda_{i}(T)$ and $\rho_{j}(T)$ commute on $\dot{\mathcal{X}}_{i}$, $\dot{\mathcal{X}}_{j}$, and $\dot{\mathcal{X}}_{i} \otimes \dot{\mathcal{X}}_{j}$, and it is trivial to see that $\lambda_{i}(T)$ and $\rho_{j}(T)$ commute on all other components of $\dot{\mathcal{X}}$.

Note that $\lambda_{i}(T)$ and $\rho_{i}(S)$ need not commute, even if $T \in \mathcal{L}_{\ell}\left(\mathcal{X}_{i}\right)$ and $S \in \mathcal{L}_{r}\left(\mathcal{X}_{i}\right)$.

3.2. Abstract Structures for Bi-Free Probability with Amalgamation. The purpose of this section is to develop an abstract notion of the pair $\left(\mathcal{L}(\mathcal{X}), E_{\mathcal{L}(\mathcal{X})}\right)$. Based on the previous section and Proposition 3.1.6. we make the following definition.

Definition 3.2.1. A $B$-B-non-commutative probability space is a triple $\left(\mathcal{A}, E_{\mathcal{A}}, \varepsilon\right)$ where $\mathcal{A}$ is a unital algebra, $\varepsilon: B \otimes B^{\text {op }} \rightarrow \mathcal{A}$ is a homomorphism such that $\left.\varepsilon\right|_{B \otimes 1_{B}}$ and $\left.\varepsilon\right|_{1_{B} \otimes B^{\text {op }}}$ are injective, and $E_{\mathcal{A}}: \mathcal{A} \rightarrow B$ is a linear map such that

for all $b_{1}, b_{2} \in B$ and $T \in \mathcal{A}$, and

$$
E_{\mathcal{A}}\left(\varepsilon\left(b_{1} \otimes b_{2}\right) T\right)=b_{1} E_{\mathcal{A}}(T) b_{2}
$$

$$
E_{\mathcal{A}}\left(T \varepsilon\left(b \otimes 1_{B}\right)\right)=E_{\mathcal{A}}\left(T \varepsilon\left(1_{B} \otimes b\right)\right)
$$

for all $b \in B$ and $T \in \mathcal{A}$.

In addition, we define the unital subalgebras $\mathcal{A}_{\ell}$ and $\mathcal{A}_{r}$ of $\mathcal{A}$ by

$$
\begin{aligned}
& \mathcal{A}_{\ell}:=\left\{T \in \mathcal{A} \mid T \varepsilon\left(1_{B} \otimes b\right)=\varepsilon\left(1_{B} \otimes b\right) T \text { for all } b \in B\right\} \\
& \mathcal{A}_{r}:=\left\{T \in \mathcal{A} \mid T \varepsilon\left(b \otimes 1_{B}\right)=\varepsilon\left(b \otimes 1_{B}\right) T \text { for all } b \in B\right\} .
\end{aligned}
$$


We call $\mathcal{A}_{\ell}$ and $\mathcal{A}_{r}$ the left and right algebras of $\mathcal{A}$ respectively.

Remark 3.2.2. If $(\mathcal{X}, \mathcal{X}, p)$ is a $B$ - $B$-bimodule with a specified $B$-vector state, we see via Proposition 3.1 .6 that $\left(\mathcal{L}(\mathcal{X}), E_{\mathcal{L}(\mathcal{X})}, \varepsilon\right)$ is a $B$-B-non-commutative probability space where $E_{\mathcal{L}(\mathcal{X})}$ is as in Definition 3.1 .5 and $\varepsilon: B \otimes B^{\text {op }} \rightarrow B$ is defined by $\varepsilon\left(b_{1} \otimes b_{2}\right)=L_{b_{1}} R_{b_{2}}$. As such, in an arbitrary $B$ - $B$-non-commutative probability space $\left(\mathcal{A}, E_{\mathcal{A}}, \varepsilon\right)$, we will often use $L_{b}$ instead of $\varepsilon(b \otimes 1)$ and $R_{b}$ instead of $\varepsilon(1 \otimes b)$, in which case $L_{b} \in \mathcal{A}_{\ell}$ and $R_{b} \in \mathcal{A}_{r}$ for all $b \in B$.

Remark 3.2.3. It may appear that Definition 3.2.1 is incompatible with the notion of a $B$-probability space in free probability: that is, a pair $(\mathcal{A}, \varphi)$ where $\mathcal{A}$ is a unital algebra containing $B$, and $\varphi: \mathcal{A} \rightarrow B$ is a linear map such that $\varphi\left(b_{1} T b_{2}\right)=b_{1} \varphi(T) b_{2}$ for all $b_{1}, b_{2} \in B$ and $T \in \mathcal{A}$ (see 3 for example). However, $\mathcal{A}$ is a $B$ - $B$-bimodule by left and right multiplication by $B$, and, by Remark 3.1.2, $\mathcal{A}$ can be made into a $B$ - $B$ bimodule with specified $B$-vector space via $\varphi$. Hence Remark $3.2 .2 \operatorname{implies} \mathcal{L}(\mathcal{A})$ is a $B$ - $B$-non-commutative probability state with

$$
E_{\mathcal{L}(\mathcal{A})}(T)=\varphi(T)
$$

for all $T \in \mathcal{L}(\mathcal{A})$. In addition, we can view $\mathcal{A}$ as a unital subalgebra of both $\mathcal{L}_{\ell}(\mathcal{A})$ and $\mathcal{L}_{r}(\mathcal{A})$ by left and right multiplication on $\mathcal{A}$ respectively.

Viewing $\mathcal{A} \subseteq \mathcal{L}_{\ell}(\mathcal{A})$, it is clear we can recover the joint $B$-moments of elements of $\mathcal{A}$ from $E_{\mathcal{L}(\mathcal{A})}$. Indeed, for $T \in \mathcal{A} \subseteq \mathcal{L}_{\ell}(\mathcal{A})$ we have

$$
E_{\mathcal{L}(\mathcal{A})}\left(L_{b_{1}} T L_{b_{2}}\right)=E_{\mathcal{L}(\mathcal{A})}\left(L_{b_{1}} T R_{b_{2}}\right)=E_{\mathcal{L}(\mathcal{A})}\left(L_{b_{1}} R_{b_{2}} T\right)=b_{1} E_{\mathcal{L}(\mathcal{A})}(T) b_{2},
$$

which is consistent with the defining property of $\varphi$.

One should note that Definition 3.2.1 differs slightly from [7, Definition 8.3]. However, given Proposition 3.1 .6 and the following result which demonstrates that a $B$ - $B$-non-commutative probability space embeds into $\mathcal{L}(\mathcal{X})$ for a $B$ - $B$-bimodule with a specified $B$-vector state $\mathcal{X}$, Definition 3.2 .1 indeed specifies the correct abstract objects to study.

Theorem 3.2.4. Let $\left(\mathcal{A}, E_{\mathcal{A}}, \varepsilon\right)$ be a B-B-non-commutative probability space. Then there exists a B-Bbimodule with a specified $B$-vector state $(\mathcal{X}, \dot{\mathcal{X}}, p)$ and a unital homomorphism $\theta: \mathcal{A} \rightarrow \mathcal{L}(\mathcal{X})$ such that

$$
\theta\left(L_{b_{1}} R_{b_{2}}\right)=L_{b_{1}} R_{b_{2}}, \quad \theta\left(\mathcal{A}_{\ell}\right) \subseteq \mathcal{L}_{\ell}(\mathcal{X}), \quad \theta\left(\mathcal{A}_{r}\right) \subseteq \mathcal{L}_{r}(\mathcal{X}), \quad \text { and } \quad E_{\mathcal{L}(\mathcal{X})}(\theta(T))=E_{\mathcal{A}}(T)
$$

for all $b_{1}, b_{2} \in B$ and $T \in \mathcal{A}$.

Proof. Consider the vector space over $\mathbb{C}$

$$
\mathcal{X}=B \oplus \mathcal{Y}
$$

where

$$
\mathcal{Y}=\operatorname{ker}\left(E_{\mathcal{A}}\right) / \operatorname{span}\left\{T L_{b}-T R_{b} \mid T \in \mathcal{A}, b \in B\right\}
$$

Note $\mathcal{Y}$ is a well-defined quotient vector space since $E_{\mathcal{A}}\left(T L_{b}-T R_{b}\right)=0$ by Definition 3.2.1 We will postpone describing the $B$ - $B$-module structure on $\mathcal{X}$ until later in the proof.

Let $q: \operatorname{ker}\left(E_{\mathcal{A}}\right) \rightarrow \mathcal{Y}$ denote the canonical quotient map. Then, for $T, A \in \mathcal{A}$ with $E_{\mathcal{A}}(A)=0$ and $b \in B$, we define $\theta(T) \in \mathcal{L}(\mathcal{X})$ by

and

$$
\theta(T)(b)=E_{\mathcal{A}}\left(T L_{b}\right) \oplus q\left(T L_{b}-L_{E_{\mathcal{A}}\left(T L_{b}\right)}\right)
$$

$$
\theta(T)(q(A))=E_{\mathcal{A}}(T A) \oplus q\left(T A-L_{E_{\mathcal{A}}(T A)}\right) .
$$

Note $\theta(T)$ is a well-defined linear operator due to the definition of $\mathcal{Y}$.

To see that $\theta$ is a homomorphism, note $\theta$ is clearly linear. To see that $\theta$ is multiplicative, fix $T, S \in \mathcal{A}$. If $b \in B$, then

Thus

$$
\theta(T)(b)=E_{\mathcal{A}}\left(T L_{b}\right) \oplus q\left(T L_{b}-L_{E\left(\mathcal{A} T L_{b}\right)}\right)
$$

$$
\begin{aligned}
\theta(S)(\theta(T)(b))= & \left.E_{\mathcal{A}}\left(S L_{E_{\mathcal{A}}\left(T L_{b}\right)}\right) \oplus q\left(S L_{E_{\mathcal{A}}\left(T L_{b}\right)}-L_{E_{\mathcal{A}}\left(S L_{E_{\mathcal{A}}}\left(T L_{b}\right)\right.}\right)\right) \\
& +E_{\mathcal{A}}\left(S\left(T L_{b}-L_{E_{\mathcal{A}}\left(T L_{b}\right)}\right)\right) \oplus q\left(S\left(T L_{b}-L_{E_{\mathcal{A}}\left(T L_{b}\right)}\right)-L_{E_{\mathcal{A}}\left(S\left(T L_{b}-L_{E_{\mathcal{A}}\left(T L_{b}\right)}\right)\right)}\right) \\
= & E_{\mathcal{A}}\left(S T L_{b}\right) \oplus q\left(S T L_{b}-L_{E_{\mathcal{A}}\left(S T L_{b}\right)}\right) \\
= & \theta(S T)(b) .
\end{aligned}
$$


Similarly, if $q(A) \in \mathcal{Y}$ then

$$
\theta(T)(q(A))=E_{\mathcal{A}}(T A) \oplus q\left(T A-L_{E_{\mathcal{A}}(T A)}\right)
$$

Thus

$$
\begin{aligned}
& \left.\theta(S)(\theta(T)(q(A)))=E_{\mathcal{A}}\left(S L_{E_{\mathcal{A}}(T A)}\right) \oplus q\left(S L_{E_{\mathcal{A}}(T A)}-L_{E_{\mathcal{A}}\left(S L_{E_{\mathcal{A}}}(T A)\right.}\right)\right) \\
& +E_{\mathcal{A}}\left(S\left(T A-L_{E_{\mathcal{A}}(T A)}\right)\right) \oplus q\left(S\left(T A-L_{E_{\mathcal{A}}(T A)}\right)-L_{E_{\mathcal{A}}\left(S\left(T A-L_{E_{\mathcal{A}}(T A)}\right)\right)}\right) \\
& =E_{\mathcal{A}}(S T A) \oplus q\left(S T A-L_{E_{\mathcal{A}}(S T A)}\right) \\
& =\theta(S T)(q(A)) \text {. }
\end{aligned}
$$

Hence $\theta$ is a homomorphism.

To make $\mathcal{X}$ a $B$ - $B$-bimodule, we define

$$
b \cdot \xi=\theta\left(L_{b}\right)(\xi) \quad \text { and } \quad \xi \cdot b=\theta\left(R_{b}\right)(\xi)
$$

for all $\xi \in \mathcal{X}$ and $b \in B$. It is clear that this is a well-defined $B$-B-bimodule structure on $\mathcal{X}$ since $\theta$ is a homomorphism.

To demonstrate that $\mathcal{X}$ is indeed a $B$ - $B$-bimodule with a specified vector state, we must show that $\mathcal{Y}$ is invariant under this $B$-B-bimodule structure, and that the $B$ - $B$-bimodule structure when restricted to $B \subseteq \mathcal{X}$ is the canonical one. If $b, b^{\prime} \in B$ and $q(A) \in \mathcal{Y}$, then

$$
\theta\left(L_{b}\right)\left(b^{\prime}\right)=E_{\mathcal{A}}\left(L_{b} L_{b^{\prime}}\right) \oplus q\left(L_{b} L_{b^{\prime}}-L_{E_{\mathcal{A}}\left(L_{b} L_{b^{\prime}}\right)}\right)=b b^{\prime} \oplus q\left(L_{b b^{\prime}}-L_{b b^{\prime}}\right)=b b^{\prime} \oplus 0
$$

and

$$
\begin{aligned}
\theta\left(L_{b}\right)(q(A)) & =E_{\mathcal{A}}\left(L_{b} A\right) \oplus q\left(L_{b} A-L_{E_{\mathcal{A}}\left(L_{b} A\right)}\right) \\
& =b E_{\mathcal{A}}(A) \oplus q\left(L_{b} A-L_{E_{\mathcal{A}}\left(L_{b} A\right)}\right)=0 \oplus q\left(L_{b} A-L_{E_{\mathcal{A}}\left(L_{b} A\right)}\right) .
\end{aligned}
$$

Similarly,

$$
\theta\left(R_{b}\right)\left(b^{\prime}\right)=E_{\mathcal{A}}\left(R_{b} L_{b^{\prime}}\right) \oplus q\left(R_{b} L_{b^{\prime}}-L_{E_{\mathcal{A}}\left(R_{b} L_{b^{\prime}}\right)}\right)=b^{\prime} b \oplus q\left(L_{b^{\prime}} R_{b}-L_{b^{\prime}} L_{b}\right)=b^{\prime} b \oplus 0
$$

and

$$
\begin{aligned}
\theta\left(R_{b}\right)(q(A)) & =E_{\mathcal{A}}\left(R_{b} A\right) \oplus q\left(R_{b} A-L_{E\left(R_{b} A\right)}\right) \\
& =E_{\mathcal{A}}(A) b \oplus q\left(R_{b} A-L_{E_{\mathcal{A}}\left(R_{b} A\right)}\right)=0 \oplus q\left(R_{b} A-L_{E_{\mathcal{A}}\left(R_{b} A\right)}\right) .
\end{aligned}
$$

Thus $\mathcal{X}$ is a $B$ - $B$-bimodule with a specified $B$-vector state.

Since $\theta$ is a homomorphism, it is clear that $\theta\left(\mathcal{A}_{\ell}\right) \subseteq \mathcal{L}_{\ell}(\mathcal{X})$ and $\theta\left(\mathcal{A}_{r}\right) \subseteq \mathcal{L}_{r}(\mathcal{X})$ due to the definition of the $B$ - $B$-bimodule structure on $\mathcal{X}$. Finally, if $T \in \mathcal{A}$ then

$$
E_{\mathcal{L}(\mathcal{X})}(\theta(T))=p\left(\theta(T)\left(1_{B} \oplus 0\right)\right)=p\left(E_{\mathcal{A}}(T) \oplus q\left(T-L_{E_{\mathcal{A}}(T)}\right)\right)=E_{\mathcal{A}}(T) .
$$

3.3. Bi-Free Families of Pairs of $B$-Faces. With the notion of a $B$ - $B$-non-commutative probability space from Definition 3.2.1, we are now able to define the main concept of this paper, following [7, Definition 8.5].

Definition 3.3.1. Let $\left(\mathcal{A}, E_{\mathcal{A}}, \varepsilon\right)$ be a $B$-B-non-commutative probability space. A pair of $B$-faces of $\mathcal{A}$ is a pair $(C, D)$ of unital subalgebras of $\mathcal{A}$ such that

$$
\varepsilon\left(B \otimes 1_{B}\right) \subseteq C \subseteq \mathcal{A}_{\ell} \quad \text { and } \quad \varepsilon\left(1_{B} \otimes B^{o p}\right) \subseteq D \subseteq \mathcal{A}_{r} .
$$

A family $\left\{\left(C_{k}, D_{k}\right)\right\}_{k \in K}$ of pair of $B$-faces of $\mathcal{A}$ is said to be bi-free with amalgamation over $B$ (or simply bi-free over $B)$ if there exist $B$-B-bimodules with specified $B$-vector states $\left\{\left(\mathcal{X}_{k}, \dot{\mathcal{X}}_{k}, p_{k}\right)\right\}_{k \in K}$ and unital homomorphisms $l_{k}: C_{k} \rightarrow \mathcal{L}_{\ell}\left(\mathcal{X}_{k}\right), r_{k}: D_{k} \rightarrow \mathcal{L}_{r}\left(\mathcal{X}_{k}\right)$ such that the joint distribution of $\left\{\left(C_{k}, D_{k}\right)\right\}_{k \in K}$ with respect to $E_{\mathcal{A}}$ is equal to the joint distribution of the images $\left\{\left(\left(\lambda_{k} \circ l_{k}\right)\left(C_{k}\right),\left(\rho_{k} \circ r_{k}\right)\left(D_{k}\right)\right)\right\}_{k \in K}$ inside $\mathcal{L}\left(*_{k \in K} \mathcal{X}_{k}\right)$ with respect to $\left.E_{\mathcal{L}\left(*_{k \in K}\right.} \mathcal{X}_{k}\right)$.

It will be an immediate consequence of Theorem 7.1.4 that the selection of representations in Definition 3.3.1 does not matter (see [7, Proposition 2.9]). Note that if $\left\{\left(C_{k}, D_{k}\right)\right\}_{k \in K}$ is bi-free over $B$, then $\left\{C_{k}\right\}_{k \in K}$ is free with amalgamation over $B$ (as is $\left\{D_{k}\right\}_{k \in K}$ ) and $C_{i}$ and $D_{j}$ commute in distributions whenever $i \neq j$.

To conclude this section, we give the following example. 
Example 3.3.2. Let $\mathfrak{M}$ be a type $\mathrm{II}_{1}$ factor with a faithful normal tracial state $\tau_{\mathfrak{M}}$ and let $\mathfrak{N}$ be a von Neumann subalgebra of $\mathfrak{M}$ containing $1_{\mathfrak{M}}$. Then there exists a canonical trace-preserving (and thus faithful) conditional expectation $E_{\mathfrak{N}}: \mathfrak{M} \rightarrow \mathfrak{N}$ given by $E_{\mathfrak{N}}(T)=P_{\mathfrak{N}} T P_{\mathfrak{N}}$ for all $T \in \mathfrak{M}$ where $P_{\mathfrak{N}}$ is the projection of $L^{2}\left(\mathfrak{M}, \tau_{\mathfrak{M}}\right)$ onto $L^{2}\left(\mathfrak{N}, \tau_{\mathfrak{M}}\right)$. Thus $\mathfrak{M}$ has a $\mathfrak{N}$ - $\mathfrak{N}$-bimodule structure with specified $\mathfrak{N}$-vector state induced by $E_{\mathfrak{N}}$ and $\mathfrak{M}$ has a canonical left and right action on this bimodule.

Suppose $\mathfrak{N}$ is a von Neumann algebra such that there exists type $\mathrm{II}_{1}$ factors $\mathfrak{M}_{1}$ and $\mathfrak{M}_{2}$ containing $\mathfrak{N}$. It is elementary to verify that $\mathfrak{M}_{1} *_{\mathfrak{N}} \mathfrak{M}_{2}$ can be made into a $\mathfrak{N}$ - $\mathfrak{N}$-non-commutative probability space with expectation $E_{\mathfrak{N}}: \mathfrak{M}_{1} *_{\mathfrak{N}} \mathfrak{M}_{2} \rightarrow \mathfrak{N}$ the canonical conditional expectation. Moreover, it is clear that $\left(\mathfrak{M}_{1}, \mathfrak{M}_{1}^{\text {op }}\right)$ and $\left(\mathfrak{M}_{2}, \mathfrak{M}_{2}^{\text {op }}\right)$ are bi-free over $\mathfrak{N}$.

\section{Operator-Valued Bi-Multiplicative Functions}

In this section, we will extend the notion of multiplicative functions (see [3, Section 2] or [5, Section 2]) in order to study $B$ - $B$-non-commutative probability spaces.

4.1. A Partial Ordering and Notation. Given $\chi:\{1, \ldots, n\} \rightarrow\{\ell, r\}$ and $\pi \in B N C(\chi)$, we consider the following additional ordering on $\{1, \ldots, n\}$.

Definition 4.1.1. Let $\chi:\{1, \ldots, n\} \rightarrow\{\ell, r\}$. The total ordering $\prec_{\chi}$ on $\{1, \ldots, n\}$ is defined as follows: for $a, b \in\{1, \ldots, n\}, a \prec_{\chi} b$ if $s_{\chi}^{-1}(a)<s_{\chi}^{-1}(b)$. Given a subset $V \subseteq\{1, \ldots, n\}$, we will say that $V$ is a $\chi$-interval if it is an interval with respect to the ordering $\prec_{\chi}$. In addition we define $\min _{\prec_{\chi}}(V)$ and $\max _{\prec_{\chi}}(V)$ to be the minimal and maximal elements of $V$ with respect to the ordering $\prec_{\chi}$.

Note that if $\pi \in B N C(\chi)$, then $\prec_{\chi}$ orders the nodes of $\pi$ from top to bottom along the left side of its bi-non-crossing diagram, then bottom to top along the right side.

Notation 4.1.2. Let $\chi:\{1, \ldots, n\} \rightarrow\{\ell, r\}$. Given a subset $S \subseteq\{1, \ldots, n\}$ we define $\left.\chi\right|_{S}: S \rightarrow\{\ell, r\}$ to be the restriction of $\chi$ to $S$. Similarly, given an $n$-tuple of objects $\left(T_{1}, \ldots, T_{n}\right)$, we define $\left.\left(T_{1}, \ldots, T_{n}\right)\right|_{S}$ to be the $|S|$-tuple where the elements in positions not indexed by an element of $S$ are removed. Finally, given $\pi \in B N C(\chi)$ such that $S$ is a union of blocks of $\pi$, we define $\left.\pi\right|_{S} \in B N C\left(\left.\chi\right|_{S}\right)$ to be the bi-non-crossing partition formed by taking the blocks of $\pi$ contained in $S$.

4.2. Definition of Bi-Multiplicative Functions. With the above definitions and notation, we can begin the necessary constructions for the operator-valued bi-free cumulants. Note simple examples follow the definition along with a heuristic version of Definition 4.2.1 will be given in Remark 4.2 .4 which should be of aid to an experienced free probabilist.

Definition 4.2.1. Let $(\mathcal{A}, E, \varepsilon)$ be a $B$ - $B$-non-commutative probability space and let

$$
\Phi: \bigcup_{n \geq 1} \bigcup_{\chi:\{1, \ldots, n\} \rightarrow\{\ell, r\}} B N C(\chi) \times \mathcal{A}_{\chi(1)} \times \cdots \times \mathcal{A}_{\chi(n)} \rightarrow B
$$

be a function that is linear in each $\mathcal{A}_{\chi(k)}$. We say that $\Phi$ is bi-multiplicative if the following four conditions hold:

(i) Let $\chi:\{1, \ldots, n\} \rightarrow\{\ell, r\}$, let $T_{k} \in \mathcal{A}_{\chi(k)}$, let $b \in B$, and let

$$
q=\max \{k \in\{1, \ldots, n\} \mid \chi(k) \neq \chi(n)\} .
$$

If $\chi(n)=\ell$ then

$$
\Phi_{1_{\chi}}\left(T_{1}, \ldots, T_{n-1}, T_{n} L_{b}\right)=\left\{\begin{array}{ll}
\Phi_{1_{\chi}}\left(T_{1}, \ldots, T_{q-1}, T_{q} R_{b}, T_{q+1}, \ldots, T_{n}\right) & \text { if } q \neq-\infty \\
\Phi_{1_{\chi}}\left(T_{1}, \ldots, T_{n-1}, T_{n}\right) b & \text { if } q=-\infty
\end{array} .\right.
$$

If $\chi(n)=r$ then

$$
\Phi_{1_{\chi}}\left(T_{1}, \ldots, T_{n-1}, T_{n} R_{b}\right)=\left\{\begin{array}{ll}
\Phi_{1_{\chi}}\left(T_{1}, \ldots, T_{q-1}, T_{q} L_{b}, T_{q+1}, \ldots, T_{n}\right) & \text { if } q \neq-\infty \\
b \Phi_{1_{\chi}}\left(T_{1}, \ldots, T_{n-1}, T_{n}\right) & \text { if } q=-\infty
\end{array} .\right.
$$

(ii) Let $\chi:\{1, \ldots, n\} \rightarrow\{\ell, r\}$, let $T_{k} \in \mathcal{A}_{\chi(k)}$, let $b \in B$, let $p \in\{1, \ldots, n\}$, and let

$$
q=\max \{k \in\{1, \ldots, n\} \mid \chi(k)=\chi(p), k<p\} .
$$




$$
\begin{aligned}
& \text { If } \chi(p)=\ell \text { then } \\
& \Phi_{1_{\chi}}\left(T_{1}, \ldots, T_{p-1}, L_{b} T_{p}, T_{p+1}, \ldots, T_{n}\right)=\left\{\begin{array}{ll}
\Phi_{1_{\chi}}\left(T_{1}, \ldots, T_{q-1}, T_{q} L_{b}, T_{q+1}, \ldots, T_{n}\right) & \text { if } q \neq-\infty \\
b \Phi_{1_{\chi}}\left(T_{1}, T_{2}, \ldots, T_{n}\right) & \text { if } q=-\infty
\end{array} .\right. \\
& \text { If } \chi(p)=r \text { then } \\
& \Phi_{1_{\chi}}\left(T_{1}, \ldots, T_{p-1}, R_{b} T_{p}, T_{p+1}, \ldots, T_{n}\right)=\left\{\begin{array}{ll}
\Phi_{1_{\chi}}\left(T_{1}, \ldots, T_{q-1}, T_{q} R_{b}, T_{q+1}, \ldots, T_{n}\right) & \text { if } q \neq-\infty \\
\Phi_{1_{\chi}}\left(T_{1}, T_{2}, \ldots, T_{n}\right) b & \text { if } q=-\infty
\end{array} .\right.
\end{aligned}
$$

(iii) Let $\chi:\{1, \ldots, n\} \rightarrow\{\ell, r\}, T_{k} \in \mathcal{A}_{\chi(k)}$, and $\pi \in B N C(\chi)$. Suppose that $V_{1}, \ldots, V_{m}$ are $\chi$-intervals which partition $\{1, \ldots, n\}$, each a union of blocks of $\pi$. Further, suppose $V_{1}, \ldots, V_{m}$ are ordered by $\prec \chi$. Then

$$
\Phi_{\pi}\left(T_{1}, \ldots, T_{n}\right)=\Phi_{\left.\pi\right|_{V_{1}}}\left(\left.\left(T_{1}, \ldots, T_{n}\right)\right|_{V_{1}}\right) \cdots \Phi_{\left.\pi\right|_{V_{m}}}\left(\left.\left(T_{1}, \ldots, T_{n}\right)\right|_{V_{m}}\right) .
$$

(iv) Let $\chi:\{1, \ldots, n\} \rightarrow\{\ell, r\}, T_{k} \in \mathcal{A}_{\chi(k)}$, and $\pi \in B N C(\chi)$. Suppose that $V$ and $W$ partition $\{1, \ldots, n\}$, each of which is a union of blocks of $\pi, V$ is a $\chi$-interval, and

$$
\min _{\prec x}(\{1, \ldots, n\}), \max _{\prec x}(\{1, \ldots, n\}) \in W .
$$

Let

$$
\theta=\max _{\prec_{\chi}}\left(\left\{k \in W \mid k \prec_{\chi} \min _{\prec_{\chi}}(V)\right\}\right) \quad \text { and } \quad \gamma=\min _{\prec_{\chi}}\left(\left\{k \in W \mid \max _{\prec_{\chi}}(V) \prec_{\chi} k\right\}\right) \text {. }
$$

Then

$$
\begin{aligned}
& \Phi_{\pi}\left(T_{1}, \ldots, T_{n}\right)= \begin{cases}\Phi_{\left.\pi\right|_{W}} \\
\Phi_{\left.\pi\right|_{W}}\left(\left.\left(T_{1}, \ldots, T_{\theta-1}, T_{\theta} L_{\Phi_{\left.\pi\right|_{V}}\left(\left.\left(T_{1}, \ldots, T_{n}\right)\right|_{V}\right)}, T_{\theta+1}, \ldots, T_{n}\right)\right|_{W}\right) & \text { if } \chi(\theta)=\ell \\
\left.\left.\left(T_{1}, \ldots, T_{\theta-1}, R_{\Phi_{\left.\pi\right|_{V}}\left(\left.\left(T_{1}, \ldots, T_{n}\right)\right|_{V}\right)} T_{\theta}, T_{\theta+1}, \ldots, T_{n}\right)\right|_{W}\right) & \text { if } \chi(\theta)=r\end{cases}
\end{aligned}
$$

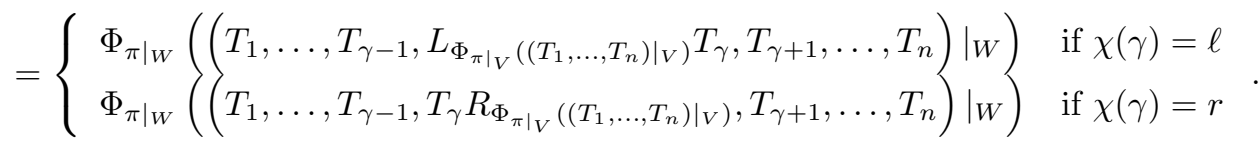

Example 4.2.2. For an example on how to apply Properties (i) and (ii) of Definition 4.2.1 consider a bimultiplicative function $\Phi$ and $\chi:\{1,2,3,4,5\} \rightarrow\{\ell, r\}$ such that $\chi^{-1}(\{\ell\})=\{1,2,4\}$ and $\chi^{-1}(\{r\})=\{3,5\}$. Then $\pi=1_{\chi}$ is represented diagrammatically as

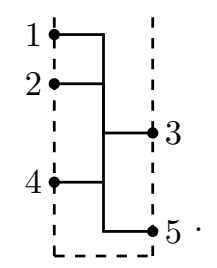

Using Properties (i) and (ii), we obtain that

$$
\Phi_{\pi}\left(T_{1}, L_{b_{1}} T_{2}, R_{b_{2}} T_{3}, T_{4}, T_{5} R_{b_{3}}\right)=\Phi_{\pi}\left(T_{1} L_{b_{1}}, T_{2}, T_{3}, T_{4} L_{b_{3}}, T_{5}\right) b_{2} .
$$

Thus one should view Properties (i) and (ii) as being able to move elements of $B$ along the dotted lines shown.

Example 4.2.3. The diagram on the left below demonstrates a bi-non-crossing partition satisfying the assumptions of Property (iii) of Definition 4.2.1 with $V_{1}=\{1,3,4\}, V_{2}=\{5,7,8\}$, and $V_{3}=\{2,6\}$. The diagram on the right below demonstrates a bi-non-crossing partition satisfying the assumptions of Property (iv) of Definition 4.2.1 with either $V=\{3,4,5,7,8\}$ and $W=\{1,2,6\}$ (in which case $\theta=1$ and $\gamma=6$ ), or 
$V=\{4,5,8\}$ and $W=\{1,2,3,6,7\}$ (in which case $\theta=3$ and $\gamma=7$ ).
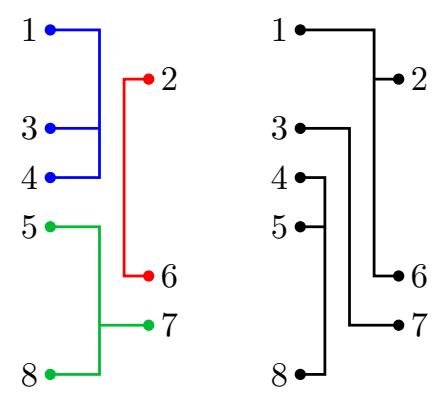

Remark 4.2.4. Although Definition 4.2.1 is cumbersome (due to the necessity of specifying cases based on whether certain terms are left or right operators), its properties can be viewed as direct analogues of those of a multiplicative map as described in [3, Section 2.2]. Indeed, for $\pi \in B N C(\chi)$ and a bi-multiplicative map $\Phi$, each expression of $\Phi_{\pi}\left(T_{1}, \ldots, T_{n}\right)$ in Definition 4.2.1 comes from viewing $s_{\chi}^{-1} \circ \pi \in N C(n)$, rearranging the $n$-tuple $\left(T_{1}, \ldots, T_{n}\right)$ to $\left(T_{s_{\chi}(1)}, \ldots, T_{s_{\chi}(n)}\right)$, replacing any occurrences of $L_{b} T_{j}, T_{j} L_{b}, R_{b} T_{j}$, and $T_{j} R_{b}$ with $b T_{j}, T_{j} b, T_{j} b$, and $b T_{j}$ respectively, applying one of the properties of a multiplicative map from [3. Section 2.2 ], and reversing the above identifications. In particular, these properties reduce to those of a multiplicative map when $\chi^{-1}(\{\ell\})=\{1, \ldots, n\}$. We use the more complex Definition 4.2.1 as it will be easier to verify for functions later on.

Since a bi-multiplicative function satisfies all of these properties, it is easy to see that if $\Phi$ is bi-multiplicative, then $\Phi_{\pi}\left(T_{1}, \ldots, T_{n}\right)$ is determined by the values

$$
\left\{\Phi_{1_{\chi^{\prime}}}\left(S_{1}, \ldots, S_{m}\right) \mid m \in \mathbb{N}, \chi^{\prime}:\{1, \ldots, m\} \rightarrow\{\ell, r\}, S_{k} \in \mathcal{A}_{\chi(k)}\right\} .
$$

There may be multiple ways to reduce $\Phi$ to an expression involving elements from the above set, but Definition 4.2.1 implies that all such reductions are equal.

Note that Definition 4.2.1 automatically implies additional properties for bi-multiplicative functions. Indeed one can either verify the following proposition via Definition 4.2.1 and casework, or can appeal to the fact that the properties of bi-multiplicative functions can be described via the properties of multiplicative functions as in Remark 4.2.4 and use the fact that multiplicative functions have additional properties (e.g. see [5, Remark 2.1.3]).

Proposition 4.2.5. Let $(\mathcal{A}, E, \varepsilon)$ be a B-B-non-commutative probability space and let

$$
\Phi: \bigcup_{n \geq 1} \bigcup_{\chi:\{1, \ldots, n\} \rightarrow\{\ell, r\}} B N C(\chi) \times \mathcal{A}_{\chi(1)} \times \cdots \times \mathcal{A}_{\chi(n)} \rightarrow B
$$

be a bi-multiplicative function. Given any $\chi:\{1, \ldots, n\} \rightarrow\{\ell, r\}, \pi \in B N C(\chi)$, and $T_{k} \in \mathcal{A}_{\chi(k)}$ Properties (i) and (ii) of Definition 4.2.1 hold when $1_{\chi}$ is replaced with $\pi$.

\section{Bi-Free Operator-Valued Moment Function is Bi-Multiplicative}

In this section, we will define the bi-free operator-valued moment function based on recursively defined functions $E_{\pi}\left(T_{1}, \ldots, T_{n}\right)$ that appear via actions on free product spaces. However, it is not immediate that it is bi-multiplicative. The proof of this result requires substantial case work, to which this section is dedicated.

5.1. Definition of the Bi-Free Operator-Valued Moment Function. We will begin with the recursive definition of expressions that appear in the operator-valued moment polynomials. These will arise in the proof of Theorem 7.1.4.

Definition 5.1.1. Let $(\mathcal{A}, E, \varepsilon)$ be a $B$-B-non-commutative probability space. For $\chi:\{1, \ldots, n\} \rightarrow\{\ell, r\}$, $\pi \in B N C(\chi)$, and $T_{1}, \ldots, T_{n} \in \mathcal{A}$, we define $E_{\pi}\left(T_{1}, \ldots, T_{n}\right) \in B$ via the following recursive process. Let $V$ be the block of $\pi$ that terminates closest to the bottom, so $\min (V)$ is largest among all blocks of $\pi$. Then:

- If $\pi$ contains exactly one block (that is, $\pi=1_{\chi}$ ), we define $E_{1_{\chi}}\left(T_{1}, \ldots, T_{n}\right)=E\left(T_{1} \cdots T_{n}\right)$. 
- If $V=\{k+1, \ldots, n\}$ for some $k \in\{1, \ldots, n-1\}$, then $\min (V)$ is not adjacent to any spines of $\pi$ and we define

$$
E_{\pi}\left(T_{1}, \ldots, T_{n}\right)=\left\{\begin{array}{ll}
E_{\left.\pi\right|_{V^{c}}}\left(T_{1}, \ldots, T_{k} L_{E_{\left.\pi\right|_{V}}\left(T_{k+1}, \ldots, T_{n}\right)}\right) & \text { if } \chi(\min (V))=\ell \\
E_{\left.\pi\right|_{V^{c}}}\left(T_{1}, \ldots, T_{k} R_{E_{\pi_{V}}}\left(T_{k+1}, \ldots, T_{n}\right)\right. & \text { if } \chi(\min (V))=r
\end{array} .\right.
$$

In the long run, it will not matter if we choose $L$ or $R$ by the first part of this recursive definition and by Definition 3.2.1

- Otherwise, $\min (V)$ is adjacent to a spine. Let $W$ denote the block of $\pi$ corresponding to the spine adjacent to $\min (V)$, and let $k$ be the first element of $W$ below where $V$ terminates - that is, $k$ is the smallest element of $W$ that is larger than $\min (V)$. We define

$$
E_{\pi}\left(T_{1}, \ldots, T_{n}\right)=\left\{\begin{array}{ll}
E_{\left.\pi\right|_{V^{c}}}\left(\left.\left(T_{1}, \ldots, T_{k-1}, L_{E_{\left.\pi\right|_{V}}\left(\left.\left(T_{1}, \ldots, T_{n}\right)\right|_{V}\right)} T_{k}, T_{k+1}, \ldots, T_{n}\right)\right|_{V^{c}}\right) & \text { if } \chi(\min (V))=\ell \\
E_{\left.\pi\right|_{V^{c}}}\left(\left.\left(T_{1}, \ldots, T_{k-1}, R_{E_{\left.\pi\right|_{V}}\left(\left.\left(T_{1}, \ldots, T_{n}\right)\right|_{V}\right)} T_{k}, T_{k+1}, \ldots, T_{n}\right)\right|_{V^{c}}\right) & \text { if } \chi(\min (V))=r
\end{array} .\right.
$$

Example 5.1.2. Let $\pi$ be the following bi-non-crossing partition.

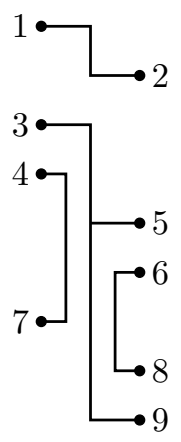

Then

$$
E_{\pi}\left(T_{1}, \ldots, T_{9}\right)=E\left(T_{1} T_{2} L_{E\left(T_{3} L_{E\left(T_{4} T_{7}\right)} T_{5} R_{E\left(T_{6} T_{8}\right)} T_{9}\right)}\right)
$$

via the following sequence of diagrams (where $X=L_{E\left(T_{3} L_{E\left(T_{4} T_{7}\right)} T_{5} R_{E\left(T_{6} T_{8}\right)} T_{9}\right)}$ ):

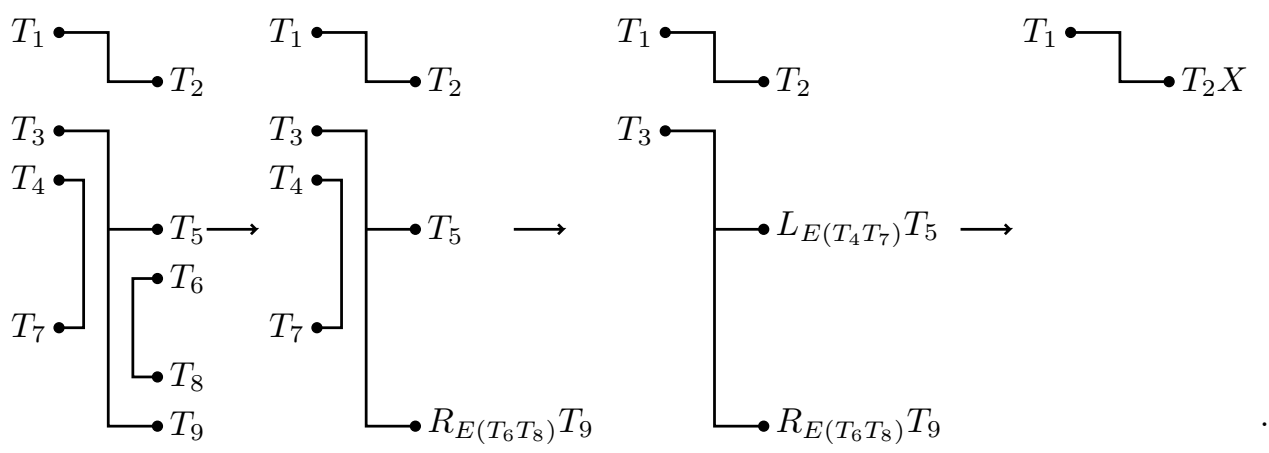

Note that the definition of $E_{\pi}\left(T_{1}, \ldots, T_{n}\right)$ is invariant under $B$-B-non-commutative probability space embeddings, such as those listed in Theorem 3.2.4. Observe that in the context of Definition 5.1.1 we ignore the notions of left and right operators. However, we are ultimately interested in the following.

Definition 5.1.3. Let $(\mathcal{A}, E, \varepsilon)$ be a $B$-B-non-commutative probability space. The bi-free operator-valued moment function

$$
\mathcal{E}: \bigcup_{n \geq 1} \bigcup_{\chi:\{1, \ldots, n\} \rightarrow\{\ell, r\}} B N C(\chi) \times \mathcal{A}_{\chi(1)} \times \cdots \times \mathcal{A}_{\chi(n)} \rightarrow B
$$

is defined by

$$
\mathcal{E}_{\pi}\left(T_{1}, \ldots, T_{n}\right)=E_{\pi}\left(T_{1}, \ldots, T_{n}\right)
$$

for each $\chi:\{1, \ldots, n\} \rightarrow\{\ell, r\}, \pi \in B N C(\chi)$, and $T_{k} \in \mathcal{A}_{\chi(k)}$.

Our next goal is the prove the following which is not apparent from Definition 5.1.1.

Theorem 5.1.4. The operator-valued bi-free moment function $\mathcal{E}$ on $\mathcal{A}$ is bi-multiplicative. 
We divide the proof of the above theorem into several lemmata, verifying various of properties from Definition 4.2.1. Properties (i) and (ii) are immediate but, unfortunately, the remaining properties are not as easily verified.

Lemma 5.1.5. The operator-valued bi-free moment function $\mathcal{E}$ satisfies Properties (i) and (ii) of Definition 4.2.1.

\subsection{Verification of Property (iii) from Definition 4.2.1 for $\mathcal{E}$.}

Lemma 5.2.1. The operator-valued bi-free moment function $\mathcal{E}$ satisfies Property (iii) of Definition 4.2.1.

Proof. We claim it suffices to consider the case that $\min _{\prec \chi}\left(V_{k}\right)$ and $\max _{\prec \chi}\left(V_{k}\right)$ are in the same block of $\pi$ for each $k \in\{1, \ldots, m\}$. Indeed, suppose the result holds with this additional assumption. Fix $V_{1}, \ldots, V_{m}$ satisfying the assumptions of Property (iii) of Definition 4.2.1. For each $k$, partition $V_{k}$ into $\chi$-intervals $V_{k, 1}, \ldots, V_{k, p_{k}}$ composed of blocks of $\pi$ ordered by $\prec_{\chi}$, with $\min _{\prec_{\chi}}\left(V_{k, q}\right) \sim_{\pi} \max _{\prec_{\chi}}\left(V_{k, q}\right)$.

Since $\bigcup_{k=1}^{m} \bigcup_{q=1}^{p_{k}} V_{k, q}$ satisfies the assumptions of Property (iii) of Definition 4.2.1 and the additional assumption, one obtains

$$
\mathcal{E}_{\pi}\left(T_{1}, \ldots, T_{n}\right)=\left(\prod_{q=1}^{p_{1}} \mathcal{E}_{\left.\pi\right|_{V_{1, q}}}\left(\left.\left(T_{1}, \ldots, T_{n}\right)\right|_{V_{1, q}}\right)\right) \ldots\left(\prod_{q=1}^{p_{m}} \mathcal{E}_{\left.\pi\right|_{V_{m}, q}}\left(\left.\left(T_{1}, \ldots, T_{n}\right)\right|_{V_{m, q}}\right)\right)
$$

In each of the above products, we write the terms from left to right in order of increasing $q$. Again, by applying the case where the additional assumption holds, one obtains that

$$
\left(\prod_{q=1}^{p_{k}} \mathcal{E}_{\left.\pi\right|_{V_{k}, q}}\left(\left.\left(T_{1}, \ldots, T_{n}\right)\right|_{V_{k, q}}\right)\right)=\mathcal{E}_{\left.\pi\right|_{V_{k}}}\left(\left.\left(T_{1}, \ldots, T_{n}\right)\right|_{V_{k}}\right)
$$

for each $k \in\{1, \ldots, m\}$. Hence we may assume that $\min _{\prec_{\chi}}\left(V_{k}\right) \sim_{\pi} \max _{\prec_{\chi}}\left(V_{k}\right)$ for each $k \in\{1, \ldots, m\}$.

To continue the proof, we proceed by induction on $m$ with the case $m=1$ being trivial. Assume Property (iii) of Definition 4.2.1 is satisfied for $\mathcal{E}$ for all smaller values of $m$. Fix $V_{1}, \ldots, V_{m}$ and note that either $1 \in V_{1}$ (i.e. $\chi(1)=\ell$ ) or $1 \in V_{m}$ (i.e. $\chi(1)=r$ ). We will treat the case when $1 \in V_{1}$; for the other case, consult a mirror. Let $V_{1}^{\prime} \subseteq V_{1}$ be the block of $\pi$ containing 1 and $\max _{{ }_{\chi}}\left(V_{1}\right)$. The proof is divided into three cases.

Case 1: $\min \left(V_{k}\right)>\max \left(V_{1}\right)$ for all $k \neq 1$. As an example of this case, consider the following diagram where $V_{1}=\{1,2,3\}, V_{2}=\{4,6\}$, and $V_{3}=\{5,7,8,9\}$.

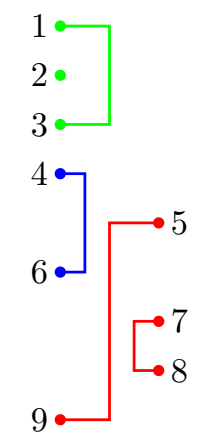

In this case, drawing a horizontal line directly beneath $\max \left(V_{1}\right)$ will hit no spines in $\pi$ and $V_{1} \subseteq \chi^{-1}(\{\ell\})$. Let $V_{1}^{\prime}=\left\{1=q_{1}<q_{2}<\cdots<q_{p}\right\}$ and $V_{0}=\bigcup_{k=2}^{m} V_{k}$. By Definition 5.1.1 we may find $b_{1}, \ldots, b_{p-1} \in B$ depending only on $\left.\left(T_{1}, \ldots, T_{n}\right)\right|_{V_{1}}$ and $\left.\pi\right|_{V_{1}}$, so that writing $T_{q_{k}}^{\prime}=T_{q_{k}} L_{b_{k}}$ we have

$$
E_{\pi}\left(T_{1}, \ldots, T_{n}\right)=E\left(T_{q_{1}}^{\prime} T_{q_{2}}^{\prime} \cdots T_{q_{p-1}}^{\prime} T_{q_{p}} L_{E_{\pi \mid V_{0}}}\left(\left.\left(T_{1}, \ldots, T_{n}\right)\right|_{V_{0}}\right)\right)=E\left(T_{q_{1}}^{\prime} T_{q_{2}}^{\prime} \cdots T_{q_{p-1}}^{\prime} T_{q_{p}} R_{E_{\pi \mid} V_{0}}\left(\left.\left(T_{1}, \ldots, T_{n}\right)\right|_{V_{0}}\right)\right) .
$$


By the assumptions in this case, each $T_{k} \in \mathcal{A}_{\ell}$ for all $k \in V_{1}^{\prime}$ and since right $B$-operators commute with elements of $\mathcal{A}_{\ell}$, we obtain

$$
\begin{aligned}
E_{\pi}\left(T_{1}, \ldots, T_{n}\right) & =E\left(T_{q_{1}}^{\prime} T_{q_{2}}^{\prime} \ldots T_{q_{p}}^{\prime} R_{\left.E_{\pi \mid}\right|_{V_{0}}}\left(\left.\left(T_{1}, \ldots, T_{n}\right)\right|_{V_{0}}\right)\right) \\
& =E\left(R_{E_{\pi \mid}}\left(\left.\left(T_{1}, \ldots, T_{n}\right)\right|_{V_{0}}\right) T_{q_{1}}^{\prime} T_{q_{2}}^{\prime} \ldots T_{q_{p}}^{\prime}\right) \\
& =E\left(T_{q_{1}}^{\prime} T_{q_{2}}^{\prime} \cdots T_{q_{p}}^{\prime}\right) E_{\left.\pi\right|_{V_{0}}}\left(\left.\left(T_{1}, \ldots, T_{n}\right)\right|_{V_{0}}\right) \\
& =E_{\left.\pi\right|_{V_{1}}}\left(\left.\left(T_{1}, \ldots, T_{n}\right)\right|_{V_{1}}\right) E_{\left.\pi\right|_{V_{0}}}\left(\left.\left(T_{1}, \ldots, T_{n}\right)\right|_{V_{0}}\right) \\
& =\mathcal{E}_{\left.\pi\right|_{V_{1}}}\left(\left.\left(T_{1}, \ldots, T_{n}\right)\right|_{V_{1}}\right) \mathcal{E}_{\left.\pi\right|_{V_{2}}}\left(\left.\left(T_{1}, \ldots, T_{n}\right)\right|_{V_{1}}\right) \cdots \mathcal{E}_{\left.\pi\right|_{V_{m}}}\left(\left.\left(T_{1}, \ldots, T_{n}\right)\right|_{V_{m}}\right)
\end{aligned}
$$

with the last step following by the inductive hypothesis.

Case 2: There exists a $k \neq 1$ such that $\min \left(V_{k}\right)<\max \left(V_{1}\right)$. In this case $V_{m}$ must terminate on the right

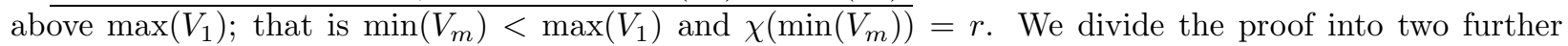
cases.

Case 2a: $\max \left(V_{1}\right)<\max \left(V_{m}\right)$. As an example of this case, consider the following diagram where $V_{1}=$ $\left\{1, \overline{3\}}, V_{2}=\{4,6,7,8,9\}\right.$, and $V_{3}=\{2,5\}$.

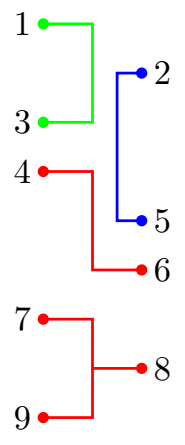

Again $V_{1} \subseteq \chi^{-1}(\{\ell\})$. With the same conventions as above, by Definition 5.1.1 we obtain

$$
E_{\pi}\left(T_{1}, \ldots, T_{n}\right)=E\left(T_{q_{1}}^{\prime} T_{q_{2}}^{\prime} \cdots T_{q_{p_{1}}}^{\prime} R_{E_{\pi \mid V_{0}}}\left(\left.\left(T_{1}, \ldots, T_{n}\right)\right|_{V_{0}}\right) T_{q_{p_{1}+1}}^{\prime} \cdots T_{q_{p_{2}}}^{\prime}\right)
$$

where $p_{1}$ is the smallest element of $V_{1}^{\prime}$ greater than $\min \left(V_{m}\right)$. By the assumptions in this case, each $T_{k} \in \mathcal{A}_{\ell}$ for all $k \in V_{1}^{\prime}$, and since right $B$-operators commute with elements of $\mathcal{A}_{\ell}$, one obtains

$$
\begin{aligned}
E_{\pi}\left(T_{1}, \ldots, T_{n}\right) & =E\left(T_{q_{1}}^{\prime} T_{q_{2}}^{\prime} \cdots T_{q_{p_{1}}}^{\prime} R_{E_{\pi \mid} V_{0}}\left(\left.\left(T_{1}, \ldots, T_{n}\right)\right|_{V_{0}}\right) T_{q_{p_{1}+1}}^{\prime} \cdots T_{q_{p_{2}}}^{\prime}\right) \\
& =E\left(R_{E_{\pi \mid}}^{\prime}\left(\left.\left(T_{1}, \ldots, T_{n}\right)\right|_{V_{0}}\right) T_{q_{1}}^{\prime} T_{q_{2}}^{\prime} \cdots T_{q_{p_{2}}}^{\prime}\right) \\
& =E\left(T_{q_{1}}^{\prime} T_{q_{2}}^{\prime} \cdots T_{q_{p_{2}}}^{\prime}\right) E_{\left.\pi\right|_{V_{0}}}\left(\left.\left(T_{1}, \ldots, T_{n}\right)\right|_{V_{0}}\right) \\
& =E_{\left.\pi\right|_{V_{1}}}\left(\left.\left(T_{1}, \ldots, T_{n}\right)\right|_{V_{1}}\right) E_{\left.\pi\right|_{V_{0}}}\left(\left.\left(T_{1}, \ldots, T_{n}\right)\right|_{V_{0}}\right) \\
& =\mathcal{E}_{\left.\pi\right|_{V_{1}}}\left(\left.\left(T_{1}, \ldots, T_{n}\right)\right|_{V_{1}}\right) \mathcal{E}_{\left.\pi\right|_{V_{2}}}\left(\left.\left(T_{1}, \ldots, T_{n}\right)\right|_{V_{1}}\right) \cdots \mathcal{E}_{\left.\pi\right|_{V_{m}}}\left(\left.\left(T_{1}, \ldots, T_{n}\right)\right|_{V_{m}}\right),
\end{aligned}
$$

with the last step following by the inductive hypothesis. 
Case 2b: $\max \left(V_{1}\right)>\max \left(V_{m}\right)$. As an example of this case, consider the following diagram where $V_{1}=$ $\left\{1, \overline{5\}}, V_{2}=\{6,8,9\}, V_{3}=\{4,7\}\right.$, and $V_{4}=\{2,3\}$.

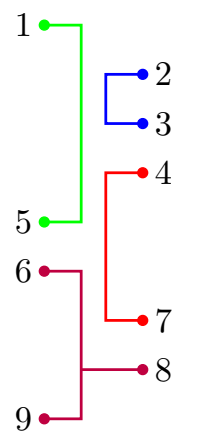

Let $V_{0}=\bigcup_{k=1}^{m-1} V_{k}$. By Definition 5.1.1 we may again find $T_{q_{1}}^{\prime}, \ldots, T_{q_{p_{1}}}^{\prime}$ and $S \in \mathcal{A}$ where $T_{k}^{\prime}$ differs from $T_{k}$ by a left multiplication operator, so that

$$
E_{\pi}\left(T_{1}, \ldots, T_{n}\right)=E\left(T_{q_{1}}^{\prime} T_{q_{2}}^{\prime} \cdots T_{q_{p_{1}}}^{\prime} R_{E_{\pi \mid V_{m}}\left(\left.\left(T_{1}, \ldots, T_{n}\right)\right|_{V_{m}}\right)} S\right)
$$

Since right $B$-operators commute with elements of $\mathcal{A}_{\ell}$, one obtains

$$
\begin{aligned}
E_{\pi}\left(T_{1}, \ldots, T_{n}\right) & =E\left(T_{q_{1}}^{\prime} T_{q_{2}}^{\prime} \cdots T_{q_{p_{1}}}^{\prime} R_{E_{\pi \mid} V_{m}}\left(\left.\left(T_{1}, \ldots, T_{n}\right)\right|_{V_{m}}\right)\right. \\
& =E\left(R_{E_{\pi \mid V_{m}}}\left(\left.\left(T_{1}, \ldots, T_{n}\right)\right|_{V_{m}}\right) T_{q_{1}}^{\prime} T_{q_{2}}^{\prime} \ldots T_{q_{p_{1}}}^{\prime} S\right) \\
& =E\left(T_{q_{1}}^{\prime} T_{q_{2}}^{\prime} \cdots T_{q_{p_{1}}}^{\prime} S\right) E_{\left.\pi\right|_{V_{m}}}\left(\left.\left(T_{1}, \ldots, T_{n}\right)\right|_{V_{m}}\right) \\
& =E_{\left.\pi\right|_{V_{0}}}\left(\left.\left(T_{1}, \ldots, T_{n}\right)\right|_{V_{0}}\right) E_{\left.\pi\right|_{V_{m}}}\left(\left.\left(T_{1}, \ldots, T_{n}\right)\right|_{V_{m}}\right) \\
& =\mathcal{E}_{\left.\pi\right|_{V_{1}}}\left(\left.\left(T_{1}, \ldots, T_{n}\right)\right|_{V_{1}}\right) \mathcal{E}_{\left.\pi\right|_{V_{2}}}\left(\left.\left(T_{1}, \ldots, T_{n}\right)\right|_{V_{1}}\right) \cdots \mathcal{E}_{\left.\pi\right|_{V_{m}}}\left(\left.\left(T_{1}, \ldots, T_{n}\right)\right|_{V_{m}}\right)
\end{aligned}
$$

with the last step following by the inductive hypothesis.

5.3. Verification of Property (iv) from Definition 4.2 .1 for $\mathcal{E}$. We begin with the following intermediate step on the way to verifying that $\mathcal{E}$ satisfies Property (iv).

Lemma 5.3.1. The operator-valued bi-free moment function $\mathcal{E}$ satisfies Property (iv) of Definition 4.2 .1 with the additional assumption that there exists a block $W_{0} \subseteq W$ of $\pi$ such that

$$
\theta, \gamma, \min _{\prec}(\{1, \ldots, n\}), \max _{\prec \chi}(\{1, \ldots, n\}) \in W_{0}
$$

Proof. We will present only the proof of the case $\chi(\theta)=\ell$ as the other cases are similar.

It is easy to see there exists a partition $V_{1}, \ldots, V_{m}$ of $V$ by $\chi$-intervals, ordered by $\prec \chi$, with $V_{k}$ a union of blocks of $\pi$ such that $\min _{\prec \chi}\left(V_{k}\right) \sim_{\pi} \max _{\prec_{\chi}}\left(V_{k}\right)$. Recall that

$$
\theta:=\max _{\prec_{\chi}}\left(\left\{k \in W \mid k \prec_{\chi} \min _{\prec_{\chi}}(V)\right\}\right) \quad \text { and } \quad \gamma:=\min _{\prec_{\chi}}\left(\left\{k \in W \mid \max _{\prec_{\chi}}(V) \prec_{\chi} k\right\}\right)
$$

and so under $\prec_{\chi}, \theta$ immediately precedes $\min _{\prec_{\chi}}\left(V_{1}\right)$ and $\gamma$ immediately follows $\max \prec_{\chi}\left(V_{m}\right)$.

The proof is now divided into three cases. 
Case 1: $\chi(\gamma)=\ell$. As an example of this case, consider the following diagram where $W=W_{0}=\{1,5,9\}$, $V_{1}=\{2,3\}, V_{2}=\{4,6,7,8\}, \theta=1$, and $\gamma=9$.

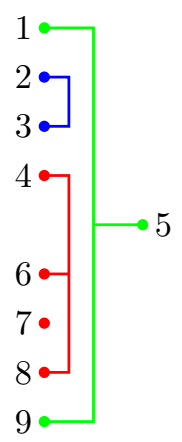

In this case $V_{k} \subseteq \chi^{-1}(\{\ell\})$ for all $k \in\{1, \ldots, m\}$. Write $X_{k}=L_{E_{\pi_{\mid} V_{k}}}\left(\left.\left(T_{1}, \ldots, T_{n}\right)\right|_{V_{k}}\right)$ and $W_{0}=\left\{q_{1}<q_{2}<\right.$ $\left.\cdots<q_{k_{m+1}}\right\}$. Then

$$
E_{\pi}\left(T_{1}, \ldots, T_{n}\right)=E\left(T_{q_{1}}^{\prime} T_{q_{2}}^{\prime} \cdots T_{q_{k_{1}}}^{\prime} X_{1} T_{q_{k_{1}+1}}^{\prime} \cdots T_{q_{k_{m}}}^{\prime} X_{m} T_{q_{k_{m}+1}}^{\prime} \cdots T_{q_{k_{m}+1}}^{\prime}\right)
$$

where $T_{k}^{\prime}$ is $T_{k}$, potentially multiplied on the left and/or right by appropriate $L_{b}$ and $R_{b}$. Here $T_{\theta}$ appears left of $X_{1}, \gamma=q_{k_{m+1}}$, and every operator between the two is either some $X_{k}$ or a right operator. Hence, by the commutation of left $B$-operators with elements of $\mathcal{A}_{r}$, we obtain

$$
E_{\pi}\left(T_{1}, \ldots, T_{n}\right)=E\left(T_{q_{1}}^{\prime} \cdots T_{q_{j-1}}^{\prime} R_{b} L_{b^{\prime}}\left(T_{\theta} X_{1} X_{2} \cdots X_{m}\right) R_{b^{\prime \prime}} T_{q_{j+1}}^{\prime} \cdots T_{q_{k_{m+1}}}^{\prime}\right)
$$

for some $b, b^{\prime}, b^{\prime \prime} \in B$. Since

$$
\mathcal{E}_{\left.\pi\right|_{V_{1}}}\left(\left.\left(T_{1}, \ldots, T_{n}\right)\right|_{V_{1}}\right) \cdots \mathcal{E}_{\left.\pi\right|_{V_{m}}}\left(\left.\left(T_{1}, \ldots, T_{n}\right)\right|_{V_{m}}\right)=\mathcal{E}_{\left.\pi\right|_{V}}\left(\left.\left(T_{1}, \ldots, T_{n}\right)\right|_{V}\right),
$$

by Lemma 5.2 .1 we have

$$
\begin{aligned}
\mathcal{E}_{\pi}\left(T_{1}, \ldots, T_{n}\right) & =E\left(T_{q_{1}}^{\prime} \cdots T_{q_{j-1}}^{\prime} R_{b} L_{b^{\prime}}\left(T_{\theta} L_{\mathcal{E}_{\left.\pi\right|_{V}}\left(\left.\left(T_{1}, \ldots, T_{n}\right)\right|_{V}\right)}\right) R_{b^{\prime \prime}} T_{q_{j+1}}^{\prime} \cdots T_{q_{k_{m}+1}}^{\prime}\right) \\
& =\mathcal{E}_{\left.\pi\right|_{W}}\left(\left.\left(T_{1}, \ldots, T_{\theta-1}, T_{\theta} L_{\mathcal{E}_{\left.\pi\right|_{V}}\left(\left.\left(T_{1}, \ldots, T_{n}\right)\right|_{V}\right)}, T_{\theta+1}, \ldots, T_{n}\right)\right|_{W}\right)
\end{aligned}
$$

where the last step follows as $\mathcal{E}_{\left.\pi\right|_{W}}$ ignores arguments corresponding to $V$.

Case 2: $\chi(\gamma)=r$ and $\theta<\gamma$. As an example of this case, consider the following diagram where $W=W_{0}=$ $\left\{1, \overline{3,6\}, V_{1}=\{2,4\}, V_{2}=\{5,8}\right\}, V_{3}=\{7,9\}, \theta=1$, and $\gamma=6$.

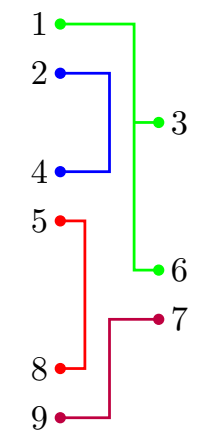

Since $\chi(\theta)=\ell$ and $\chi(\gamma)=r$ there exists a $p \in\{1, \ldots, m\}$ such that $\min \left(V_{k}\right)>\gamma$ if and only if $k>p$, $V_{k} \subseteq \chi^{-1}(\{\ell\})$ for all $k<p$, and $\chi\left(\min \left(V_{p}\right)\right)=\ell$. Let

$$
Y=\left\{\begin{array}{ll}
\bigcup_{k \geq p} V_{k} & \text { if } p<m \text { and } \min \left(V_{p+1}\right)<\max \left(V_{p}\right) \\
\bigcup_{k>p} V_{k} & \text { otherwise }
\end{array} .\right.
$$

For example, $Y=\{5,7,8,9\}$ in the above diagram. 
Let us deal with the first possible case for $Y$, with the second following similarly. In this case $V_{k} \subseteq \chi^{-1}(\{\ell\})$ for all $k \in\{1, \ldots, p-1\}$. Write $X_{k}=L_{E_{\pi \mid} V_{k}}\left(\left.\left(T_{1}, \ldots, T_{n}\right)\right|_{V_{k}}\right)$ and $W_{0}=\left\{q_{1}<q_{2}<\cdots<q_{k_{p+1}}\right\}$. Then

$$
E_{\pi}\left(T_{1}, \ldots, T_{n}\right)=E\left(T_{q_{1}}^{\prime} \cdots T_{q_{k_{1}}}^{\prime} X_{1} T_{q_{k_{1}+1}}^{\prime} \cdots T_{q_{k_{p-1}}}^{\prime} X_{p-1} T_{q_{k_{p-1}+1}}^{\prime} \cdots T_{q_{k_{p}}}^{\prime} L_{E_{\pi \mid}(}\left(\left.\left(T_{1}, \ldots, T_{n}\right)\right|_{Y}\right) T_{q_{k_{p}+1}}^{\prime} \cdots T_{q_{k_{p+1}}}^{\prime}\right)
$$

where $T_{k}^{\prime}$ is $T_{k}$, potentially multiplied on the left and/or right by appropriate $L_{b}$ and $R_{b}$. Here $T_{\theta}$ appears left of $X_{1}, \gamma=q_{k_{p+1}}$, and every operator between the two is either some $X_{k}, L_{E_{\pi_{Y}}\left(\left.\left(T_{1}, \ldots, T_{n}\right)\right|_{Y}\right)}$, or a right operator. Hence, by the commutation of left $B$-operators with elements of $\mathcal{A}_{r}$, we obtain

$$
E_{\pi}\left(T_{1}, \ldots, T_{n}\right)=E\left(T_{q_{1}}^{\prime} \cdots T_{q_{j-1}}^{\prime} R_{b} L_{b^{\prime}}\left(T_{\theta} X_{1} \cdots X_{p} L_{E_{\pi \mid}\left(\left.\left(T_{1}, \ldots, T_{n}\right)\right|_{Y}\right)}\right) R_{b^{\prime \prime}} T_{q_{j+1}}^{\prime} \cdots T_{q_{k_{p+1}}}^{\prime}\right)
$$

for some $b, b^{\prime}, b^{\prime \prime} \in B$. Since

$$
E_{\left.\pi\right|_{V_{1}}}\left(\left.\left(T_{1}, \ldots, T_{n}\right)\right|_{V_{1}}\right) \cdots E_{\left.\pi\right|_{V_{p}}}\left(\left.\left(T_{1}, \ldots, T_{n}\right)\right|_{V_{p}}\right) E_{\left.\pi\right|_{Y}}\left(\left.\left(T_{1}, \ldots, T_{n}\right)\right|_{Y}\right)=E_{\left.\pi\right|_{V}}\left(\left.\left(T_{1}, \ldots, T_{n}\right)\right|_{V}\right),
$$

by Lemma 5.2 .1 we have

$$
\begin{aligned}
\mathcal{E}_{\pi}\left(T_{1}, \ldots, T_{n}\right) & =E\left(T_{q_{1}}^{\prime} \cdots T_{q_{j-1}}^{\prime} R_{b} L_{b^{\prime}}\left(T_{\theta} L_{\mathcal{E}_{\left.\pi\right|_{V}}\left(\left.\left(T_{1}, \ldots, T_{n}\right)\right|_{V}\right)}\right) R_{b^{\prime \prime}} T_{q_{j+1}}^{\prime} \cdots T_{q_{k_{p+1}}}^{\prime}\right) \\
& =\mathcal{E}_{\left.\pi\right|_{W}}\left(\left.\left(T_{1}, \ldots, T_{\theta-1}, T_{\theta} L_{\mathcal{E}_{\left.\pi\right|_{V}}\left(\left.\left(T_{1}, \ldots, T_{n}\right)\right|_{V}\right)}, T_{\theta+1}, \ldots, T_{n}\right)\right|_{W}\right),
\end{aligned}
$$

where the last step follows as $\mathcal{E}_{\left.\pi\right|_{W}}$ ignores arguments corresponding to $V$.

Case 3: $\chi(\gamma)=r$ and $\theta>\gamma$. This case is a reflection of the second case plus a small argument. Since $\chi(\bar{\theta})=\ell$ and $\chi(\gamma)=r$ there exists a $p \in\{1, \ldots, m\}$ such that $\min \left(V_{k}\right)>\gamma$ if and only $k<p, V_{k} \subseteq \chi^{-1}(\{r\})$ for all $k>p$, and $\chi\left(\min \left(V_{p}\right)\right)=r$. Let

$$
Y=\left\{\begin{array}{ll}
\bigcup_{k \leq p} V_{k} & \text { if } p>1 \text { and } \min \left(V_{p-1}\right)<\max \left(V_{p}\right) \\
\bigcup_{k<p} V_{k} & \text { otherwise }
\end{array} .\right.
$$

Let us deal with the second possible case for $Y$, with the first following similarly. In this case $V_{k} \subseteq \chi^{-1}(\{\ell\})$ for all $k \in\{p+1, \ldots, m\}$. Write $X_{k}=R_{E_{\pi \mid V_{k}}}\left(\left.\left(T_{1}, \ldots, T_{n}\right)\right|_{V_{k}}\right)$ and $W_{0}=\left\{q_{1}<q_{2}<\cdots<q_{k_{m-p+2}}\right\}$. Then

$$
E_{\pi}\left(T_{1}, \ldots, T_{n}\right)=E\left(T_{q_{1}}^{\prime} \cdots T_{q_{k_{1}}}^{\prime} X_{m} T_{q_{k_{1}+1}}^{\prime} \cdots T_{q_{k_{m-p+1}}}^{\prime} X_{p} T_{q_{k_{m-p+1}+1}}^{\prime} \cdots T_{q_{k_{m-p+2}}}^{\prime} R_{E_{\pi \mid Y}\left(\left.\left(T_{1}, \ldots, T_{n}\right)\right|_{Y}\right)}\right)
$$

where $T_{k}^{\prime}$ is $T_{k}$, potentially multiplied on the left and/or right by appropriate $L_{b}$ and $R_{b}$. Here $\theta=q_{k_{m-p+2}}$, $T_{\gamma}$ occurs left of $X_{m}$, and every operator between the two is either some $X_{k}$ or a left operator. Hence, by the commutation of right $B$-operators with elements of $\mathcal{A}_{\ell}$, one obtains

$$
\begin{aligned}
& E_{\pi}\left(T_{1}, \ldots, T_{n}\right)=E\left(T_{q_{1}}^{\prime} \cdots T_{q_{k_{m-p+2}-1}}^{\prime} L_{b} T_{\theta} X_{m} \cdots X_{p} R_{E_{\left.\pi\right|_{Y}}\left(\left.\left(T_{1}, \ldots, T_{n}\right)\right|_{Y}\right)}\right)
\end{aligned}
$$

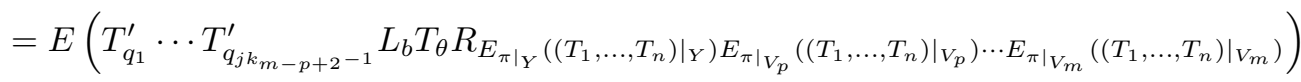

$$
\begin{aligned}
& =E\left(T_{q_{1}}^{\prime} \cdots T_{q_{j k_{m-p+2}-1}}^{\prime} L_{b} T_{\theta} L_{E_{\left.\pi\right|_{Y}}}\left(\left.\left(T_{1}, \ldots, T_{n}\right)\right|_{Y}\right) E_{\left.\pi\right|_{V_{p}}}\left(\left.\left(T_{1}, \ldots, T_{n}\right)\right|_{V_{p}}\right) \cdots E_{\left.\pi\right|_{V_{m}}}\left(\left.\left(T_{1}, \ldots, T_{n}\right)\right|_{V_{m}}\right)\right)
\end{aligned}
$$

for some $b \in B$. Since

$$
E_{\left.\pi\right|_{Y}}\left(\left.\left(T_{1}, \ldots, T_{n}\right)\right|_{Y}\right) E_{\left.\pi\right|_{V_{p}}}\left(\left.\left(T_{1}, \ldots, T_{n}\right)\right|_{V_{p}}\right) \cdots E_{\left.\pi\right|_{V_{m}}}\left(\left.\left(T_{1}, \ldots, T_{n}\right)\right|_{V_{m}}\right)=E_{\left.\pi\right|_{V}}\left(\left.\left(T_{1}, \ldots, T_{n}\right)\right|_{V}\right)
$$

by Lemma 5.2.1, we have

$$
\begin{aligned}
\mathcal{E}_{\pi}\left(T_{1}, \ldots, T_{n}\right) & =E\left(T_{q_{1}}^{\prime} \cdots T_{q_{j k_{m-p+2}-1}}^{\prime} L_{b^{\prime}} T_{\theta} L_{\mathcal{E}_{\left.\pi\right|_{V}}\left(\left.\left(T_{1}, \ldots, T_{n}\right)\right|_{V}\right)}\right) \\
& =\mathcal{E}_{\left.\pi\right|_{W}}\left(\left.\left(T_{1}, \ldots, T_{\theta-1}, T_{\theta} L_{\mathcal{E}_{\left.\pi\right|_{V}}\left(\left.\left(T_{1}, \ldots, T_{n}\right)\right|_{V}\right)}, T_{\theta+1}, \ldots, T_{n}\right)\right|_{W}\right)
\end{aligned}
$$

where the last step follows as $\mathcal{E}_{\left.\pi\right|_{W}}$ ignores arguments corresponding to $V$.

In addition to the above, we will need to verify slightly enhanced versions of Properties (i) and (ii) of Definition 4.2 .1 for $\mathcal{E}$.

Lemma 5.3.2. The operator-valued bi-free moment function $\mathcal{E}$ satisfies the $q=-\infty$ case of Property (i) of Definition 4.2.1 when $1_{\chi}$ is replaced with an arbitrary $\pi \in B N C(\chi)$. 
Proof. We will assume $\chi(n)=\ell$ as the case where $\chi(n)=r$ will follow mutatis mutandis. In the case $\chi(n)=\ell$, it is easy to see that $\chi \equiv \ell$. Let $V_{1}, \ldots, V_{m}$ be a partition of $\{1, \ldots, n\}$ into $\chi$-intervals ordered by $\prec_{\chi}$ with each $V_{k}$ a union of blocks of $\pi$, such that $\min _{\prec_{\chi}}\left(V_{k}\right) \sim_{\pi} \max _{\prec_{\chi}}\left(V_{k}\right)$, and let $V_{k}^{\prime} \in \pi$ be the block containing them. By Lemma 5.2.1, we may reduce to the case where $m=1$.

Writing $V_{1}^{\prime}=\left\{1=q_{1}<q_{2}<\cdots<q_{p+1}=n\right\}$, for some $b_{j} \in B$ depending only on $\left.\left(T_{1}, \ldots, T_{n}\right)\right|_{\left(V_{1}^{\prime}\right)^{c}}$ and on $\pi$,

$$
E_{\pi}\left(T_{1}, \ldots, T_{n}\right)=E\left(T_{q_{1}} L_{b_{1}} T_{q_{2}} L_{b_{2}} \cdots T_{q_{p}} L_{b_{p}} T_{q_{p+1}}\right) .
$$

Hence, by the commutation of right $B$-operators with elements of $\mathcal{A}_{\ell}$, we obtain

$$
\begin{aligned}
\mathcal{E}_{\pi}\left(T_{1}, \ldots, T_{n}\right) b & =E\left(T_{q_{1}} L_{b_{1}} T_{q_{2}} L_{b_{2}} \cdots T_{q_{p}} L_{b_{p}} T_{q_{p+1}}\right) b \\
& =E\left(R_{b} T_{q_{1}} L_{b_{1}} T_{q_{2}} L_{b_{2}} \cdots T_{q_{p}} L_{b_{p}} T_{q_{p+1}}\right) \\
& =E\left(T_{q_{1}} L_{b_{1}} T_{q_{2}} L_{b_{2}} \cdots T_{q_{p}} L_{b_{p}} T_{q_{p+1}} R_{b}\right) \\
& =E\left(T_{q_{1}} L_{b_{1}} T_{q_{2}} L_{b_{2}} \cdots T_{q_{p}} L_{b_{p}} T_{q_{p+1}} L_{b}\right) \\
& =\mathcal{E}_{\pi}\left(T_{1}, \ldots, T_{n} L_{b}\right) .
\end{aligned}
$$

Lemma 5.3.3. The operator-valued bi-free moment function $\mathcal{E}$ satisfies the $q=-\infty$ case of Property (ii) of Definition 4.2 .1 when $1_{\chi}$ is replaced with an arbitrary $\pi \in B N C(\chi)$.

Proof. We will assume $\chi(p)=\ell$ as the case where $\chi(p)=r$ will follow mutatis mutandis. Let $V_{1}, \ldots, V_{m}$ be a partition of $\{1, \ldots, n\}$ into $\chi$-intervals ordered by $\prec_{\chi}$ with each $V_{k}$ a union of blocks of $\pi$, such that $\min _{\prec_{\chi}}\left(V_{k}\right) \sim_{\pi} \max _{\prec_{\chi}}\left(V_{k}\right)$, and let $V_{k}^{\prime} \in \pi$ be the block containing them. By definitions, notice $p \in V_{1}^{\prime}$. Thus Lemma 5.2.1 implies we may reduce to the case where $m=1$.

Writing $V_{1}^{\prime}=\left\{q_{1}<q_{2}<\cdots<q_{k}\right\}$, for some $b_{j} \in B$ depending only on $\left.\left(T_{1}, \ldots, T_{n}\right)\right|_{\left(V_{1}^{\prime}\right)^{c}}$ and on $\pi$, and for some $S \in \mathcal{A}$,

$$
\mathcal{E}_{\pi}\left(T_{1}, \ldots, T_{n}\right)=E\left(T_{q_{1}} R_{b_{1}} \cdots T_{q_{z}} R_{b_{z}} T_{p} S\right)
$$

for some $z<k$. Hence, by the commutation of left $B$-operators with elements of $\mathcal{A}_{r}$, we obtain

$$
\begin{aligned}
b \mathcal{E}_{\pi}\left(T_{1}, \ldots, T_{n}\right) & =b E\left(T_{q_{1}} R_{b_{1}} \cdots T_{q_{z}} R_{b_{z}^{\prime}} T_{p} S\right) \\
& =E\left(L_{b} T_{q_{1}} R_{b_{1}} \cdots T_{q_{z}} R_{b_{z}} T_{p} S\right) \\
& =E\left(T_{q_{1}} R_{b_{1}} \cdots T_{q_{z}} R_{b_{z}} L_{b} T_{p} S\right) \\
& =\mathcal{E}_{\pi}\left(T_{1}, \ldots, T_{p-1}, L_{b} T_{p}, T_{p+1}, \ldots, T_{n}\right) .
\end{aligned}
$$

Lemma 5.3.4. The operator-valued bi-free moment function $\mathcal{E}$ satisfies Property (iv) of Definition 4.2.1.

Proof. Again, only the proof of the first case where $\chi(\theta)=\ell$ will be presented. We proceed by induction on $m$, the number of blocks $U \in \pi$ with

$$
U \subseteq W, \quad \min _{\prec_{\chi}}(U) \prec_{\chi} \min _{\prec_{\chi}}(V), \quad \text { and } \quad \max _{\prec_{\chi}}(V) \prec_{\chi} \max _{\prec_{\chi}}(U) .
$$

However, we first must deal with the case $m=0$. Let

$$
W_{1}=\{k \in\{1, \ldots, n\} \mid k \preceq \chi \theta\} \quad \text { and } \quad W_{2}=\{k \in\{1, \ldots, n\} \mid \gamma \preceq \chi k\} .
$$

By the assumptions on $W$, both $W_{1}$ and $W_{2}$ are $\chi$-intervals that are unions of blocks of $\pi$ such that $W=W_{1} \sqcup W_{2}$, and $W_{1} \subseteq \chi^{-1}(\ell)$. Therefore by Lemmata 5.2.1 and 5.3.2.

$$
\begin{aligned}
\mathcal{E}_{\pi}\left(T_{1}, \ldots, T_{n}\right) & =\mathcal{E}_{\left.\pi\right|_{W_{1}}}\left(\left.\left(T_{1}, \ldots, T_{n}\right)\right|_{W_{1}}\right) \mathcal{E}_{\left.\pi\right|_{V}}\left(\left.\left(T_{1}, \ldots, T_{n}\right)\right|_{V}\right) \mathcal{E}_{\left.\pi\right|_{W_{2}}}\left(\left.\left(T_{1}, \ldots, T_{n}\right)\right|_{W_{2}}\right) \\
& =\mathcal{E}_{\left.\pi\right|_{W_{1}}}\left(\left.\left(T_{1}, \ldots, T_{\theta-1}, T_{\theta} L_{\mathcal{E}_{\pi \mid}}\left(\left.\left(T_{1}, \ldots, T_{n}\right)\right|_{V}\right), T_{\theta+1}, \ldots, T_{n}\right)\right|_{W_{1}}\right) \mathcal{E}_{\left.\pi\right|_{W_{2}}}\left(\left.\left(T_{1}, \ldots, T_{n}\right)\right|_{W_{2}}\right) \\
& =\mathcal{E}_{\left.\pi\right|_{W}}\left(\left.\left(T_{1}, \ldots, T_{\theta-1}, T_{\theta} L_{\mathcal{E}_{\left.\pi\right|_{V}}}\left(\left.\left(T_{1}, \ldots, T_{n}\right)\right|_{V}\right), T_{\theta+1}, \ldots, T_{n}\right)\right|_{W}\right) .
\end{aligned}
$$

Note that we would invoke Lemma 5.3.3 instead of 5.3.2 in the case $\chi(\theta)=r$.

For the base case of the inductive argument, suppose $m=1$. Let

$$
\begin{aligned}
& \alpha_{1}=\min _{\prec_{\chi}}\left(W_{0}\right), \\
& \beta_{1}=\max _{\prec x}\left(W_{0}\right), \quad \text { and } \\
& \alpha_{2}=\max _{\prec \chi}\left(\left\{k \in W_{0} \mid k \preceq \chi \theta\right\}\right), \\
& \beta_{2}=\min _{\prec \chi}\left(\left\{k \in W_{0} \mid \gamma \preceq_{\chi} k\right\}\right) .
\end{aligned}
$$


Furthermore, let

$$
\begin{aligned}
& W_{1}^{\prime}=\left\{k \in\{1, \ldots, n\} \mid k \prec_{\chi} \alpha_{1}\right\}, \\
& W_{2}^{\prime}=\left\{k \in\{1, \ldots, n\} \mid \beta_{1} \prec_{\chi} k\right\}, \\
& W_{1}^{\prime \prime}=\left\{k \in\{1, \ldots, n\} \mid \alpha_{2} \prec k \preceq_{\chi} \theta\right\} \text {, } \\
& W_{2}^{\prime \prime}=\left\{k \in\{1, \ldots, n\} \mid \gamma \preceq_{\chi} k \prec_{\chi} \beta_{2}\right\} \text {. }
\end{aligned}
$$

Therefore, if

$$
\begin{array}{rlrl}
X_{1}^{\prime} & =\mathcal{E}_{\left.\pi\right|_{W_{1}^{\prime}}}\left(\left.\left(T_{1}, \ldots, T_{n}\right)\right|_{W_{1}^{\prime}}\right), & X_{2}^{\prime} & =\mathcal{E}_{\left.\pi\right|_{W_{2}^{\prime}}}\left(\left.\left(T_{1}, \ldots, T_{n}\right)\right|_{W_{2}^{\prime}}\right), \\
X_{1}^{\prime \prime}=\mathcal{E}_{\left.\pi\right|_{W_{1}^{\prime \prime}}}\left(\left.\left(T_{1}, \ldots, T_{n}\right)\right|_{W_{1}^{\prime \prime}}\right), & X_{2}^{\prime \prime}=\mathcal{E}_{\left.\pi\right|_{W_{2}^{\prime \prime}}}\left(\left.\left(T_{1}, \ldots, T_{n}\right)\right|_{W_{2}^{\prime \prime}}\right)
\end{array}
$$

then by Lemmata 5.2.1 and 5.3.1.

$$
\begin{aligned}
& \mathcal{E}_{\pi}\left(T_{1}, \ldots, T_{n}\right)=X_{1}^{\prime} \mathcal{E}_{\left.\pi\right|_{W_{0} \cup W_{1}^{\prime \prime} \cup W_{2}^{\prime \prime} \cup V}}\left(\left.\left(T_{1}, \ldots, T_{n}\right)\right|_{W_{0} \cup W_{1}^{\prime \prime} \cup W_{2}^{\prime \prime} \cup V}\right) X_{2}^{\prime} \\
&=X_{1}^{\prime} \mathcal{E}_{\left.\pi\right|_{W_{0}}}\left(\left(T_{1}, \ldots, T_{\alpha_{2}-1},\left.T_{\alpha_{2}} L_{\mathcal{E}_{\pi \mid} W_{1}^{\prime \prime} \cup W_{2}^{\prime \prime} \cup V}\left(\left.\left(T_{1}, \ldots, T_{n}\right)\right|_{W_{1}^{\prime \prime} \cup W_{2}^{\prime \prime} \cup V}, T_{\alpha_{2}+1}, \ldots, T_{n}\right)\right|_{W_{0}}\right) X_{2}^{\prime}\right. \\
&=X_{1}^{\prime} \mathcal{E}_{\left.\pi\right|_{W_{0}}}\left(\left(T_{1}, \ldots, T_{\alpha_{2}-1}, T_{\alpha_{2}} L_{X_{1}^{\prime \prime} \mathcal{E}_{\pi \mid}}\left(\left.\left(T_{1}, \ldots, T_{n}\right)\right|_{V}\right) X_{2}^{\prime \prime}\right.\right. \\
&\left.\left., T_{\alpha_{2}+1}, \ldots, T_{n}\right)\left.\right|_{W_{0}}\right) X_{2}^{\prime} .
\end{aligned}
$$

If $W_{1}^{\prime \prime}$ is empty, then $\alpha_{2}=\theta$ and

$$
T_{\alpha_{2}} L_{X_{1}^{\prime \prime} \mathcal{E}_{\left.\pi\right|_{V}}\left(\left.\left(T_{1}, \ldots, T_{n}\right)\right|_{V}\right) X_{2}^{\prime \prime}}=T_{\theta} L_{\mathcal{E}_{\pi \mid V}}\left(\left.\left(T_{1}, \ldots, T_{n}\right)\right|_{V}\right) L_{X_{2}^{\prime \prime}}
$$

On the other hand, if $W_{1}^{\prime \prime}$ is non-empty, then Lemma 5.3 .2 implies that

$$
X_{1}^{\prime \prime} \mathcal{E}_{\left.\pi\right|_{V}}\left(\left.\left(T_{1}, \ldots, T_{n}\right)\right|_{V}\right)=\mathcal{E}_{\left.\pi\right|_{W_{1}^{\prime \prime}}}\left(\left.\left(T_{1}, \ldots, T_{\theta-1}, T_{\theta} L_{\mathcal{E}_{\left.\pi\right|_{V}}\left(\left.\left(T_{1}, \ldots, T_{n}\right)\right|_{V}\right)}, T_{\theta+1}, \ldots, T_{n}\right)\right|_{W_{1}^{\prime \prime}}\right)
$$

The result follows now from Lemmata 5.2.1 and 5.3.1 in the direction opposite the above.

Inductively, suppose that the result holds when $m \geq 1$. Suppose $W$ contains blocks $W_{0}, \ldots, W_{m}$ of $\pi$ which satisfy the above inequalities. Without loss of generality, we may assume that

$$
\min _{\prec_{\chi}}\left(W_{0}\right) \prec_{\chi} \cdots \prec_{\chi} \min _{\prec_{\chi}}\left(W_{m}\right) .
$$

Note that as $\pi$ is bi-non-crossing, this implies

$$
\max _{\prec \chi}\left(W_{m}\right) \prec_{\chi} \cdots \prec_{\chi} \max _{\prec_{\chi}}\left(W_{0}\right)
$$

Let $\alpha_{1}, \alpha_{2}, \beta_{1}, \beta_{2}, W_{1}^{\prime}, W_{2}^{\prime}, X_{1}^{\prime}$, and $X_{2}^{\prime}$ be as above. Hence applying Lemmata 5.2.1 and 5.3.1 once again gives

$$
\begin{aligned}
& \mathcal{E}_{\pi}\left(T_{1}, \ldots, T_{n}\right) \\
& \quad=X_{1}^{\prime} \mathcal{E}_{\left.\pi\right|_{\left(W_{1}^{\prime} \cup W_{2}^{\prime}\right)^{c}}}\left(\left.\left(T_{1}, \ldots, T_{n}\right)\right|_{\left(W_{1}^{\prime} \cup W_{2}^{\prime}\right)^{c}}\right) X_{2}^{\prime} \\
& \quad=X_{1}^{\prime} \mathcal{E}_{\left.\pi\right|_{W_{0}}}\left(\left(T_{1}, \ldots, T_{\alpha_{2}-1},\left.T_{\alpha_{2}} L_{\mathcal{E}_{\pi \mid}\left(W_{0} \cup W_{1}^{\prime} \cup W_{2}^{\prime}\right)^{c}}\left(\left.\left(T_{1}, \ldots, T_{n}\right)\right|_{\left.\left(W_{0} \cup W_{1}^{\prime} \cup W_{2}^{\prime}\right)^{c}\right)}, T_{\alpha_{2}+1}, \ldots, T_{n}\right)\right|_{W_{0}}\right) X_{2}^{\prime}\right.
\end{aligned}
$$

Now, by the inductive hypothesis, we see that

$$
\begin{aligned}
\mathcal{E}_{\left.\pi\right|_{\left(W_{0} \cup W_{1}^{\prime} \cup W_{2}^{\prime}\right)^{c}}}\left(\left.\left(T_{1}, \ldots, T_{n}\right)\right|_{\left(W_{0} \cup W_{1}^{\prime} \cup W_{2}^{\prime}\right)^{c}}\right) \\
\quad=\mathcal{E}_{\left.\pi\right|_{\left(W_{0} \cup W_{1}^{\prime} \cup W_{2}^{\prime}\right)^{c} \backslash V}}\left(\left.\left(T_{1}, \ldots, T_{\theta-1}, T_{\theta} L_{\mathcal{E}_{\pi \mid}}\left(\left.\left(T_{1}, \ldots, T_{n}\right)\right|_{V}\right), T_{\theta+1}, \ldots, T_{n}\right)\right|_{\left.\left(W_{0} \cup W_{1}^{\prime} \cup W_{2}^{\prime}\right)^{c} \backslash V\right) .}\right.
\end{aligned}
$$

Hence, by substituting this expression into the above computation and applying Lemmata 5.2 .1 and 5.3 .1 in the opposite order, the inductive step is complete.

With this result, the proof of Theorem 5.1.4 is complete. 


\section{Operator-Valued Bi-Free Cumulants}

6.1. Cumulants via Convolution. Following [5, Definition 2.1.6], we begin with a definition of operatorvalued convolution.

Definition 6.1.1. Let $(\mathcal{A}, E, \varepsilon)$ be a $B$ - $B$-non-commutative probability space, let

$$
\Phi: \bigcup_{n \geq 1} \bigcup_{\chi:\{1, \ldots, n\} \rightarrow\{\ell, r\}} B N C(\chi) \times \mathcal{A}_{\chi(1)} \times \cdots \times \mathcal{A}_{\chi(n)} \rightarrow B
$$

and let $f \in I A(B N C)$. We define the convolution of $\Phi$ and $f$, denoted $\Phi * f$, by

$$
(\Phi * f)_{\pi}\left(T_{1}, \ldots, T_{n}\right):=\sum_{\substack{\sigma \in B N C(\chi) \\ \sigma \leq \pi}} \Phi_{\sigma}\left(T_{1}, \ldots, T_{n}\right) f(\sigma, \pi)
$$

for all $\chi:\{1, \ldots, n\} \rightarrow\{\ell, r\}, \pi \in B N C(\chi)$, and $T_{k} \in \mathcal{A}_{\chi(k)}$.

Remark 6.1.2. One can check that if $\Phi$ is as above and $f, g \in I A(B N C)$ then $(\Phi * f) * g=\Phi *(f * g)$.

Definition 6.1.3. Let $(\mathcal{A}, E, \varepsilon)$ be a $B$-B-non-commutative probability space and let $\mathcal{E}$ be the operatorvalued bi-free moment function on $\mathcal{A}$. The operator-valued bi-free cumulant function is the function

$$
\kappa: \bigcup_{n \geq 1} \bigcup_{\chi:\{1, \ldots, n\} \rightarrow\{\ell, r\}} B N C(\chi) \times \mathcal{A}_{\chi(1)} \times \cdots \times \mathcal{A}_{\chi(n)} \rightarrow B
$$

defined by

$$
\kappa:=\mathcal{E} * \mu_{B N C}
$$

Note for $\chi:\{1, \ldots, n\} \rightarrow\{\ell, r\}, \pi \in B N C(\chi)$, and $T_{k} \in \mathcal{A}_{\chi(k)}$ that

$$
\kappa_{\pi}\left(T_{1}, \ldots, T_{n}\right)=\sum_{\sigma \leq \pi} \mathcal{E}_{\sigma}\left(T_{1}, \ldots, T_{n}\right) \mu_{B N C}(\sigma, \pi) \quad \text { and } \quad \mathcal{E}_{\pi}\left(T_{1}, \ldots, T_{n}\right)=\sum_{\sigma \leq \pi} \kappa_{\sigma}\left(T_{1}, \ldots, T_{n}\right)
$$

6.2. Convolution Preserves Bi-Multiplicativity. It is now straightforward to demonstrate the operatorvalued bi-free cumulant function is bi-multiplicative.

Theorem 6.2.1. Let $(\mathcal{A}, E, \varepsilon)$ be a $B$-B-non-commutative probability space, let

$$
\Phi: \bigcup_{n \geq 1 \chi:\{1, \ldots, n\} \rightarrow\{\ell, r\}} \bigcup B N C(\chi) \times \mathcal{A}_{\chi(1)} \times \cdots \times \mathcal{A}_{\chi(n)} \rightarrow B
$$

and let $f \in I A(B N C)$. If $\Phi$ is bi-multiplicative and $f$ is multiplicative, then $\Phi * f$ is bi-multiplicative.

Corollary 6.2.2. The operator-valued bi-free cumulant function is bi-multiplicative.

Proof of Theorem 6.2.1. Clearly $(\Phi * f)_{\pi}$ is linear in each entry. Furthermore, Proposition 4.2.5 establishes that $\Phi * f$ satisfies Properties (i) and (ii) of Definition 4.2.1. Thus it remains to verify Properties (iii) and (iv).

Suppose the hypotheses of Property (iii). We see that

$$
\begin{aligned}
(\Phi * f)_{\pi} & \left(T_{1}, \ldots, T_{n}\right) \\
& =\sum_{\sigma \leq \pi} \Phi_{\sigma}\left(T_{1}, \ldots, T_{n}\right) f(\sigma, \pi) \\
& =\sum_{\sigma \leq \pi} \Phi_{\left.\sigma\right|_{V_{1}}}\left(\left.\left(T_{1}, \ldots, T_{n}\right)\right|_{V_{1}}\right) \cdots \Phi_{\left.\sigma\right|_{V_{m}}}\left(\left.\left(T_{1}, \ldots, T_{n}\right)\right|_{V_{m}}\right) f\left(\left.\sigma\right|_{V_{1}},\left.\pi\right|_{V_{1}}\right) \cdots f\left(\left.\sigma\right|_{V_{m}},\left.\pi\right|_{V_{m}}\right) \\
& =(\Phi * f)_{\left.\pi\right|_{V_{1}}}\left(\left.\left(T_{1}, \ldots, T_{n}\right)\right|_{V_{1}}\right) \cdots(\Phi * f)_{\left.\pi\right|_{V_{m}}}\left(\left.\left(T_{1}, \ldots, T_{n}\right)\right|_{V_{m}}\right)
\end{aligned}
$$

using the bi-multiplicativity of $\Phi$ and the multiplicativity of $f$. 
To see Property (iv) holds, note under the hypotheses of its initial case,

$$
\begin{aligned}
(\Phi * f)_{\pi} & \left(T_{1}, \ldots, T_{n}\right) \\
& =\sum_{\sigma \leq \pi} \Phi_{\sigma}\left(T_{1}, \ldots, T_{n}\right) f(\sigma, \pi) \\
& =\sum_{\sigma \leq \pi} \Phi_{\left.\sigma\right|_{W}}\left(\left.\left(T_{1}, \ldots, T_{\theta-1}, T_{\theta} L_{\Phi_{\left.\sigma\right|_{V}}\left(\left.\left(T_{1}, \ldots, T_{n}\right)\right|_{V}\right)}, T_{\theta+1}, \ldots, T_{n}\right)\right|_{W}\right) f\left(\left.\sigma\right|_{V},\left.\pi\right|_{V}\right) f\left(\left.\sigma\right|_{W},\left.\pi\right|_{W}\right) \\
& =\sum_{\sigma \leq \pi} \Phi_{\left.\sigma\right|_{W}}\left(\left.\left(T_{1}, \ldots, T_{\theta-1}, T_{\theta} L_{\Phi_{\left.\sigma\right|_{V}}}\left(\left.\left(T_{1}, \ldots, T_{n}\right)\right|_{V}\right) f\left(\left.\sigma\right|_{V},\left.\pi\right|_{V}\right), T_{\theta+1}, \ldots, T_{n}\right)\right|_{W}\right) f\left(\left.\sigma\right|_{W},\left.\pi\right|_{W}\right) \\
& =(\Phi * f)_{\left.\pi\right|_{W}}\left(\left.\left(T_{1}, \ldots, T_{\theta-1}, T_{\theta} L_{\Phi_{\left.\sigma\right|_{V}}}\left(\left.\left(T_{1}, \ldots, T_{n}\right)\right|_{V}\right), T_{\theta+1}, \ldots, T_{n}\right)\right|_{W}\right),
\end{aligned}
$$

again by the corresponding properties of $\Phi$ and $f$. The proof of the remaining three statements in Property (iv) is identical.

6.3. Bi-Moment and Bi-Cumulant Functions. Inspired by [5, Section 3.2], we define the formal classes of bi-moment and bi-cumulant functions and give an important relation between them. It follows readily that the operator-valued bi-free moment and cumulant functions on a $B$ - $B$-non-commutative probability space are examples of these types of functions, respectively.

We begin with the following useful notation.

Notation 6.3.1. Let $\chi:\{1, \ldots, n\} \rightarrow\{\ell, r\}, \pi \in B N C(\chi)$, and $q \in\{1, \ldots, n\}$. We denote by $\left.\chi\right|_{\backslash q}$ the restriction of $\chi$ to the set $\{1, \ldots, n\} \backslash\{q\}$. In addition, if $q \neq n$, we define $\left.\pi\right|_{q=q+1} \in B N C\left(\left.\chi\right|_{\backslash q}\right)$ to be the bi-non-crossing partition which results from identifying $q$ and $q+1$ in $\pi$ (i.e. if $q$ and $q+1$ are in the same block as $\pi$ then $\left.\pi\right|_{q=q+1}$ is obtained from $\pi$ by just removing $q$ from the block in which $q$ occurs, while if $q$ and $q+1$ are in different blocks, $\left.\pi\right|_{q=q+1}$ is obtained from $\pi$ by merging the two blocks and then removing q).

Definition 6.3.2. Let $(\mathcal{A}, E, \varepsilon)$ be a $B$ - $B$-non-commutative probability space and let

$$
\Phi: \bigcup_{n \geq 1} \bigcup_{\chi:\{1, \ldots, n\} \rightarrow\{\ell, r\}} B N C(\chi) \times \mathcal{A}_{\chi(1)} \times \cdots \times \mathcal{A}_{\chi(n)} \rightarrow B
$$

be bi-multiplicative. We say that $\Phi$ is a bi-moment function if whenever $\chi:\{1, \ldots, n\} \rightarrow\{\ell, r\}$ is such that there exists a $q \in\{1, \ldots, n-1\}$ with $\chi(q)=\chi(q+1)$, then

$$
\Phi_{1_{\chi}}\left(T_{1}, \ldots, T_{n}\right)=\Phi_{1_{\chi \backslash \backslash q}}\left(T_{1}, \ldots, T_{q-1}, T_{q} T_{q+1}, T_{q+2}, \ldots, T_{n}\right)
$$

for all $T_{k} \in \mathcal{A}_{\chi(k)}$. Similarly, we say that $\Phi$ is a bi-cumulant function if whenever $\chi:\{1, \ldots, n\} \rightarrow\{\ell, r\}$ and $\pi \in B N C(\chi)$ are such that there exists a $q \in\{1, \ldots, n-1\}$ with $\chi(q)=\chi(q+1)$, then

$$
\Phi_{1_{\chi \backslash \backslash q}}\left(T_{1}, \ldots, T_{q-1}, T_{q} T_{q+1}, T_{q+2}, \ldots, T_{n}\right)=\Phi_{1_{\chi}}\left(T_{1}, \ldots, T_{n}\right)+\sum_{\substack{\pi \in B N C(\chi) \\|\pi|=2, q \varkappa_{\pi} q+1}} \Phi_{\pi}\left(T_{1}, \ldots, T_{n}\right)
$$

for all $T_{k} \in \mathcal{A}_{\chi(k)}$.

Remark 6.3.3. The operator-valued bi-free moment function $\mathcal{E}$ is a bi-moment function.

Before relating the notions of bi-moment and bi-cumulant functions, we note the following alternate formulations.

Lemma 6.3.4. Let $(\mathcal{A}, E, \varepsilon)$ be a $B$-B-non-commutative probability space and let

$$
\Phi: \bigcup_{n \geq 1} \bigcup_{\chi:\{1, \ldots, n\} \rightarrow\{\ell, r\}} B N C(\chi) \times \mathcal{A}_{\chi(1)} \times \cdots \times \mathcal{A}_{\chi(n)} \rightarrow B
$$

be bi-multiplicative. Then $\Phi$ is a bi-moment function if and only if whenever $\chi:\{1, \ldots, n\} \rightarrow\{\ell, r\}$ and $\pi \in B N C(\chi)$ are such that there exists a $q \in\{1, \ldots, n-1\}$ with $\chi(q)=\chi(q+1)$ and $q \sim_{\pi} q+1$, then

$$
\Phi_{\pi}\left(T_{1}, \ldots, T_{n}\right)=\Phi_{\left.\pi\right|_{q=q+1}}\left(T_{1}, \ldots, T_{q-1}, T_{q} T_{q+1}, T_{q+2}, \ldots T_{n}\right)
$$


for all $T_{k} \in \mathcal{A}_{\chi(k)}$. Similarly, $\Phi$ is a bi-cumulant function if and only if whenever $\chi:\{1, \ldots, n\} \rightarrow\{\ell, r\}$ is such that there exists a $q \in\{1, \ldots, n-1\}$ with $\chi(q)=\chi(q+1)$, we have

$$
\Phi_{\pi}\left(T_{1}, \ldots, T_{q-1}, T_{q} T_{q+1}, T_{q+2}, \ldots T_{n}\right)=\sum_{\substack{\left.\sigma \in B N C(\chi) \\ \sigma\right|_{q=q+1}=\pi}} \Phi_{\sigma}\left(T_{1}, \ldots, T_{n}\right)
$$

for all $\pi \in B N C\left(\left.\chi\right|_{\backslash q}\right)$.

To establish the lemma, one uses bi-multiplicativity to reduce to the case of full partitions and then applies Definition 6.3.2.

Theorem 6.3.5. Let $(\mathcal{A}, E, \varepsilon)$ be a $B$-B-non-commutative probability space and let

$$
\Phi, \Psi: \bigcup_{n \geq 1} \bigcup_{\chi:\{1, \ldots, n\} \rightarrow\{\ell, r\}} B N C(\chi) \times \mathcal{A}_{\chi(1)} \times \cdots \times \mathcal{A}_{\chi(n)} \rightarrow B
$$

be related by the formulae

$$
\Phi=\Psi * \zeta_{B N C}, \quad \text { or equivalently } \quad \Psi=\Phi * \mu_{B N C} .
$$

Then $\Phi$ is a bi-moment function if and only if $\Psi$ is a bi-cumulant function.

Proof. To begin, note $\Phi$ is bi-multiplicative if and only if $\Psi$ is bi-multiplicative by Theorem 6.2.1

Suppose $\Psi$ is a bi-cumulant function. If $\chi:\{1, \ldots, n\} \rightarrow\{\ell, r\}$ is such that there exists a $q \in\{1, \ldots, n-1\}$ with $\chi(q)=\chi(q+1)$, then for all $T_{k} \in \mathcal{A}_{\chi(k)}$

$$
\begin{aligned}
& \Phi_{1_{\chi \backslash \backslash q}}\left(T_{1}, \ldots, T_{q-1}, T_{q} T_{q+1}, T_{q+2}, \ldots, T_{n}\right)=\sum_{\pi \in B N C\left(\chi \backslash_{\backslash q}\right)} \Psi_{\pi}\left(T_{1}, \ldots, T_{q-1}, T_{q} T_{q+1}, T_{q+2}, \ldots, T_{n}\right) \\
& =\sum_{\pi \in B N C\left(\chi \backslash_{\backslash q}\right)} \sum_{\substack{\left.\sigma \in B N C(\chi) \\
\sigma\right|_{q=q+1}=\pi}} \Psi_{\sigma}\left(T_{1}, \ldots, T_{n}\right) \\
& =\sum_{\sigma \in B N C(\chi)} \Psi_{\sigma}\left(T_{1}, \ldots, T_{n}\right) \\
& =\Phi_{1_{\chi}}\left(T_{1}, \ldots, T_{n}\right) \text {. }
\end{aligned}
$$

Thus $\Phi$ is a bi-moment function.

For the other direction, suppose $\Phi$ is a bi-moment function. Let $\chi:\{1, \ldots, n\} \rightarrow\{\ell, r\}$. We will proceed by induction on $n$. If $n=1$, there is nothing to check. If $n=2$, then

$$
\Psi_{\left.1_{\chi}\right|_{1=2}}\left(T_{1} T_{2}\right)=\Phi_{1_{\left.\chi\right|_{1=2}}}\left(T_{1} T_{2}\right)=\Phi_{1_{\chi}}\left(T_{1}, T_{2}\right)=\Psi_{1_{\chi}}\left(T_{1}, T_{2}\right)+\Psi_{0_{\chi}}\left(T_{1}, T_{2}\right)
$$

as required.

Suppose that the formula from Definition 6.3.2 holds for $n-1$. Then using the induction hypothesis and bi-multiplicativity of $\Psi$, we see for all $\pi \in B N C\left(\left.\chi\right|_{\backslash q}\right) \backslash\left\{1_{\left.\chi\right|_{\backslash q}}\right\}$ that

$$
\Psi_{\pi}\left(T_{1}, \ldots, T_{q-1}, T_{q} T_{q+1}, T_{q+2}, \ldots, T_{n}\right)=\sum_{\substack{\left.\sigma \in B N C(\chi) \\ \sigma\right|_{q=q+1}=\pi}} \Psi_{\sigma}\left(T_{1}, \ldots, T_{n}\right)
$$


Hence

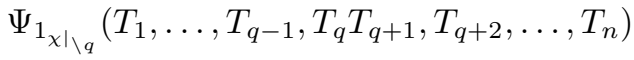

$$
\begin{aligned}
& =\Phi_{1_{\chi \backslash \backslash q}}\left(T_{1}, \ldots, T_{q-1}, T_{q} T_{q+1}, T_{q+2}, \ldots, T_{n}\right)-\sum_{\substack{\pi \in B N C\left(\chi \backslash \backslash q \\
\pi \neq 1 \times 1_{\backslash q}\right.}} \Psi_{\pi}\left(T_{1}, \ldots, T_{q-1}, T_{q} T_{q+1}, T_{q+2}, \ldots T_{n}\right) \\
& =\Phi_{1_{\chi}}\left(T_{1}, \ldots, T_{n}\right)-\sum_{\substack{\pi \in B N C\left(\left.\chi\right|_{\backslash q}\right) \\
\pi \neq 1_{\chi \backslash \backslash q}}} \sum_{\substack{\left.\sigma \in B N C(\chi) \\
\sigma\right|_{q=q+1}=\pi}} \Psi_{\sigma}\left(T_{1}, \ldots, T_{n}\right) \\
& =\sum_{\sigma \in B N C(\chi)} \Psi_{\sigma}\left(T_{1}, \ldots, T_{n}\right)-\sum_{\substack{\left.\sigma \in B N C(\chi) \\
\sigma\right|_{q=q+1} \neq\left. 1_{\chi \mid}\right|_{\backslash q}}} \Psi_{\sigma}\left(T_{1}, \ldots, T_{n}\right) \\
& =\sum_{\substack{\left.\sigma \in B N C(\chi) \\
\sigma\right|_{q=q+1}=1_{\chi \backslash \backslash q}}} \Psi_{\sigma}\left(T_{1}, \ldots, T_{n}\right)
\end{aligned}
$$

Corollary 6.3.6. The operator-valued bi-free cumulant function $\kappa$ is a bi-cumulant function.

6.4. Vanishing of Operator-Valued Bi-Free Cumulants. The following demonstrates, like with classical and free cumulants, that operator-valued bi-free cumulants of order at least two vanish provided at least one entry is an element of $B$.

Proposition 6.4.1. Let $(\mathcal{A}, E, \varepsilon)$ be a $B$-B-non-commutative probability space, $\chi:\{1, \ldots, n\} \rightarrow\{\ell, r\}$ with $n \geq 2$, and $T_{k} \in \mathcal{A}_{\chi(k)}$. If there exist $q \in\{1, \ldots, n\}$ and $b \in B$ such that $T_{q}=L_{b}$ if $\chi(q)=\ell$ or $T_{q}=R_{b}$ if $\chi(q)=r$, then

$$
\kappa\left(T_{1}, \ldots, T_{n}\right)=0
$$

Proof. The base case can be readily established by computing directly the cumulants of order two.

For the inductive step, suppose the result holds for all $\chi:\{1, \ldots, k\} \rightarrow\{\ell, r\}$ with $k<n$. Fix $\chi:$ $\{1, \ldots, n\} \rightarrow\{\ell, r\}$ and $T_{k} \in \mathcal{A}_{\chi(k)}$. Suppose that for some $q \in\{1, \ldots, n\}$ we have $\chi(q)=\ell$ and $T_{q}=L_{b}$ with $b \in B$, as the argument for the right side is similar.

Let

$$
p=\max \{k \in\{1, \ldots, n\} \mid \chi(k)=\ell, k<q\} .
$$

The proof is now divided into two cases.

Case 1: $p \neq-\infty$. In this case we notice that

$$
\begin{aligned}
\kappa_{1_{\chi}}\left(T_{1}, \ldots, T_{n}\right) & =\mathcal{E}_{1_{\chi}}\left(T_{1}, \ldots, T_{n}\right)-\sum_{\substack{\pi \in B N C(\chi) \\
\pi \neq 1_{\chi}}} \kappa_{\pi}\left(T_{1}, \ldots, T_{n}\right) \\
& =\mathcal{E}_{1_{\chi}}\left(T_{1}, \ldots, T_{n}\right)-\sum_{\substack{\pi \in B N C(\chi) \\
\{q\} \in \pi}} \kappa_{\pi}\left(T_{1}, \ldots, T_{p-1}, T_{p}, T_{p+1}, \ldots, T_{q-1}, L_{b}, T_{q+1}, \ldots, T_{n}\right) \\
& =\mathcal{E}_{1_{\chi}}\left(T_{1}, \ldots, T_{n}\right)-\sum_{\sigma \in B N C\left(\chi \backslash_{\backslash q}\right)} \kappa_{\sigma}\left(T_{1}, \ldots, T_{p-1}, T_{p} L_{b}, T_{p+1}, \ldots, T_{q-1}, T_{q+1}, \ldots, T_{n}\right),
\end{aligned}
$$

by induction and Proposition 4.2.5. Since

$$
\begin{aligned}
\mathcal{E}_{1_{\chi}}\left(T_{1}, \ldots, T_{n}\right) & =E\left(T_{1} \cdots T_{n}\right) \\
& =E\left(T_{1} \cdots T_{q-1} L_{b} T_{q+1} \cdots T_{n}\right) \\
& =E\left(T_{1} \cdots T_{p-1} L_{b} T_{p+1} \cdots T_{n}\right) \\
& =\sum_{\sigma \in B N C\left(\chi \backslash_{\backslash q}\right)} \kappa_{\sigma}\left(T_{1}, \ldots, T_{p-1}, T_{p} L_{b}, T_{p+1}, \ldots, T_{q-1}, T_{q+1}, \ldots, T_{n}\right),
\end{aligned}
$$

the proof is complete in this case. 
Case 2: $p=-\infty$. In this case, notice that

$$
\begin{aligned}
\kappa_{1_{\chi}}\left(T_{1}, \ldots, T_{n}\right) & =\mathcal{E}_{1_{\chi}}\left(T_{1}, \ldots, T_{n}\right)-\sum_{\substack{\pi \in B N C(\chi) \\
\pi \neq 1_{\chi}}} \kappa_{\pi}\left(T_{1}, \ldots, T_{n}\right) \\
& =\mathcal{E}_{1_{\chi}}\left(T_{1}, \ldots, T_{n}\right)-\sum_{\substack{\pi \in B N C(\chi) \\
\{q\} \in \pi}} \kappa_{\pi}\left(T_{1}, \ldots, T_{q-1}, L_{b}, T_{q+1}, \ldots, T_{n}\right) \\
& =\mathcal{E}_{1_{\chi}}\left(T_{1}, \ldots, T_{n}\right)-\sum_{\sigma \in B N C(\chi \backslash \backslash q)} b \kappa_{\sigma}\left(T_{1}, \ldots, T_{q-1}, T_{q+1}, \ldots, T_{n}\right),
\end{aligned}
$$

by induction and Proposition 4.2.5. Since

$$
\begin{aligned}
\mathcal{E}_{1_{\chi}}\left(T_{1}, \ldots, T_{n}\right) & =E\left(T_{1} \cdots T_{n}\right) \\
& =E\left(T_{1} \cdots T_{q-1} L_{b} T_{q+1} \cdots T_{n}\right) \\
& =E\left(L_{b} T_{1} \cdots T_{q-1} T_{q+1} \cdots T_{n}\right) \\
& =b E\left(T_{1} \cdots T_{q-1} T_{q+1} \cdots T_{n}\right) \\
& =\sum_{\sigma \in B N C\left(\chi \backslash_{\backslash q}\right)} b \kappa_{\sigma}\left(T_{1}, \ldots, T_{q-1}, T_{q+1}, \ldots, T_{n}\right),
\end{aligned}
$$

the proof is complete in this case as well.

\section{Universal Moment Polynomials for Bi-Free Families with Amalgamation}

In this section, we will generalize [1, Corollary 4.1.2] to demonstrate that algebras will be bi-free with amalgamation over $B$ if and only if certain moment expressions hold. To do so, we will need to extend the definition of $E_{\pi}\left(T_{1}, \ldots, T_{n}\right)$ to an extension of the shaded $L R$ diagrams as defined in Section 2.1.

\subsection{Equivalence of Bi-Free with Amalgamation and Universal Moment Polynomials.}

Definition 7.1.1. Let $\chi:\{1, \ldots, n\} \rightarrow\{\ell, r\}$ and let $\epsilon:\{1, \ldots, n\} \rightarrow K$. Let $L R_{k}^{\text {lat }}(\chi, \epsilon)$ denote the closure of $L R_{k}(\chi, \epsilon)$ under lateral refinement. Observe that every diagram in $L R_{k}^{\text {lat }}(\chi, \epsilon)$ still has $k$ strings reaching its top, as lateral refinements may only introduce cuts between ribs. Given $D_{1}, D_{2} \in L R_{k}^{\text {lat }}(\chi, \epsilon)$, we write $D_{2} \leq_{\text {lat }} D_{1}$ if $D_{2}$ can be obtained by laterally refining $D_{1}$. Finally, we denote

$$
L R^{\text {lat }}(\chi, \epsilon):=\bigcup_{k \geq 0} L R_{k}^{\text {lat }}(\chi, \epsilon) .
$$

Definition 7.1.2. Let $\left\{\left(\mathcal{X}_{k}, \dot{\mathcal{X}}_{k}, p_{k}\right)\right\}_{k \in K}$ be $B$-B-bimodules with specified $B$-vector states, let $\lambda_{k}$ and $\rho_{k}$ be as defined in Construction 3.1.7, and let $\mathcal{X}=\left(*_{B}\right)_{k \in K} \mathcal{X}_{k}$. Let $\chi:\{1, \ldots, n\} \rightarrow\{\ell, r\}, \epsilon:\{1, \ldots, n\} \rightarrow K$, $D \in L R^{\text {lat }}(\chi, \epsilon)$, and $T_{k} \in \mathcal{L}_{\chi(k)}\left(\mathcal{X}_{\epsilon(k)}\right)$. Define $\mu_{k}\left(T_{k}\right)=\lambda_{\epsilon(k)}\left(T_{k}\right)$ if $\chi(k)=\ell$ and $\mu_{k}\left(T_{k}\right)=\rho_{\epsilon(k)}\left(T_{k}\right)$ if $\chi(k)=r$. We define $E_{D}\left(\mu_{1}\left(T_{1}\right), \ldots, \mu_{n}\left(T_{n}\right)\right)$ recursively as follows: Apply the same recursive process as in Definition 5.1.1 until every block of $\pi$ has a spine reaching the top. If every block of $D$ has a spine reaching the top, enumerate them from left to right according to their spines as $V_{1}, \ldots, V_{m}$ with $V_{j}=\left\{k_{j, 1}<\cdots<k_{j, q_{j}}\right\}$, and set

$$
E_{D}\left(\mu_{1}\left(T_{1}\right), \ldots, \mu_{n}\left(T_{n}\right)\right)=\left[\left(1-p_{\epsilon\left(k_{1,1}\right)}\right) T_{k_{1,1}} \cdots T_{k_{1, q_{1}}} 1_{B}\right] \otimes \cdots \otimes\left[\left(1-p_{\epsilon\left(k_{m, 1}\right)}\right) T_{k_{m, 1}} \cdots T_{k_{m, q_{m}}} 1_{B}\right] .
$$

Lemma 7.1.3. With the notation as in Definition 7.1.2.

$$
\mu_{1}\left(T_{1}\right) \cdots \mu_{n}\left(T_{n}\right) 1_{B}=\sum_{k=0}^{n} \sum_{D \in L R_{k}^{\text {lat }}(\chi, \epsilon)}\left[\sum_{\substack{D^{\prime} \in L R_{k}(\chi, \epsilon) \\ D^{\prime} \geq \text { at } D}}(-1)^{|D|-\left|D^{\prime}\right|}\right] E_{D}\left(\mu_{1}\left(T_{1}\right), \ldots, \mu_{n}\left(T_{n}\right)\right),
$$

where $|D|$ and $\left|D^{\prime}\right|$ denote the number of blocks of $D$ and $D^{\prime}$ respectively. In particular,

$$
E_{\mathcal{L}(\mathcal{X})}\left(\mu_{1}\left(T_{1}\right) \mu_{2}\left(T_{2}\right) \cdots \mu_{n}\left(T_{n}\right)\right)=\sum_{\pi \in B N C(\chi)}\left[\sum_{\substack{\sigma \in B N C(\chi) \\ \pi \leq \sigma \leq \epsilon}} \mu_{B N C}(\pi, \sigma)\right] \mathcal{E}_{\pi}\left(\mu_{1}\left(T_{1}\right), \ldots, \mu_{n}\left(T_{n}\right)\right) .
$$


Proof. To begin, note that the second claim follows from the first by Definition 7.1.2 and by Theorem 2.3.5. To prove the main claim we will proceed by induction on the number of operators $n$. The case $n=1$ is trivial.

For the inductive step, we will assume that $\chi(1)=\ell$ as the proof in the case $\chi(1)=r$ will follow by similar arguments. Let $\chi_{0}=\left.\chi\right|_{\{2, \ldots, n\}}$ and $\epsilon_{0}=\left.\epsilon\right|_{\{2, \ldots, n\}}$. By induction, we obtain that

$$
\mu_{2}\left(T_{2}\right) \cdots \mu_{n}\left(T_{n}\right) 1_{B}=\sum_{k=0}^{n-1} \sum_{D_{0} \in L R_{k}^{\text {lat }}\left(\chi_{0}, \epsilon_{0}\right)}\left[\sum_{\substack{D_{0}^{\prime} \in L R_{k}\left(\chi_{0}, \epsilon_{0}\right) \\ D_{0}^{\prime} \geq 1 \text { lat } D_{0}}}(-1)^{\left|D_{0}\right|-\left|D_{0}^{\prime}\right|}\right] E_{D_{0}}\left(\mu_{2}\left(T_{2}\right), \ldots, \mu_{n}\left(T_{n}\right)\right) .
$$

The result will follow by applying $\lambda_{1}\left(T_{1}\right)$ to each $E_{D_{0}}\left(\mu_{2}\left(T_{2}\right), \ldots, \mu_{n}\left(T_{n}\right)\right)$, checking the correct terms appear, collecting the same terms, and verifying the coefficients are correct.

Fix $D_{0} \in L R_{k}^{\text {lat }}\left(\chi_{0}, \epsilon_{0}\right)$. We can write

$$
E_{D_{0}}\left(\mu_{2}\left(T_{2}\right), \ldots, \mu_{n}\left(T_{n}\right)\right)=\left[\left(1-p_{\epsilon\left(k_{1}\right)}\right) S_{1} 1_{B}\right] \otimes \cdots \otimes\left[\left(1-p_{\epsilon\left(k_{m}\right)}\right) S_{m} 1_{B}\right]
$$

for some operators $S_{p} \in \operatorname{alg}\left(\lambda_{k_{p}}\left(\mathcal{L}_{\ell}\left(\mathcal{X}_{k_{p}}\right)\right), \rho_{k_{p}}\left(\mathcal{L}_{r}\left(\mathcal{X}_{k_{p}}\right)\right)\right)$. To demonstrate the correct terms appear, we divide the analysis into three cases.

Case 1: $m=0$. In this case $E_{D_{0}}\left(\mu_{2}\left(T_{2}\right), \ldots, \mu_{n}\left(T_{n}\right)\right)=b \in B$. As such, we see that

$$
\lambda_{\epsilon(1)}\left(T_{1}\right) E_{D_{0}}\left(\mu_{2}\left(T_{2}\right), \ldots, \mu_{n}\left(T_{n}\right)\right)=E\left(T_{1}\right) b \oplus\left[\left(1-p_{\epsilon(1)}\right) T_{1} b\right]
$$

If $D_{1}$ is the $L R$-diagram obtained from $D_{0}$ by placing a node shaded $\epsilon(1)$ at the top and $D_{2}$ is the $L R$ diagram obtained from $D_{0}$ by placing a node $\epsilon(1)$ at the top and drawing a spine from this node to the top, then since

$$
E\left(\mu_{1}\left(T_{1}\right) L_{b}\right)=E\left(\mu_{1}\left(T_{1}\right) R_{b}\right)=E\left(R_{b} \mu_{1}\left(T_{1}\right)\right)=E\left(T_{1}\right) b
$$

and

$$
T_{1} b=T_{1} R_{b} 1_{B}=T_{1} L_{b} 1_{B}
$$

one easily sees that

$$
E\left(T_{1}\right) b=E_{D_{1}}\left(\mu_{1}\left(T_{1}\right), \mu_{2}\left(T_{2}\right), \ldots, \mu_{n}\left(T_{n}\right)\right) \quad \text { and } \quad\left(1-p_{\epsilon(1)}\right) T_{1} b=E_{D_{2}}\left(\mu_{1}\left(T_{1}\right), \mu_{2}\left(T_{2}\right), \ldots, \mu_{n}\left(T_{n}\right)\right) .
$$

Case 2: $m \neq 0$ and $\epsilon(1) \neq \epsilon\left(k_{1}\right)$. In this case, $\left(1-p_{\epsilon\left(k_{1}\right)}\right) S_{1} 1_{B}$ is in a space orthogonal to $\dot{\mathcal{X}}_{\epsilon(1)}$. Thus

$$
\begin{aligned}
\lambda_{\epsilon(1)}\left(T_{1}\right) E_{D_{0}}\left(\mu_{2}\left(T_{2}\right), \ldots, \mu_{n}\left(T_{n}\right)\right)= & \left(\left[L_{E\left(T_{1}\right)}\left(1-p_{\epsilon\left(k_{1}\right)}\right) S_{1} 1_{B}\right] \otimes \cdots \otimes\left[\left(1-p_{\epsilon\left(k_{m}\right)}\right) S_{m} 1_{B}\right]\right) \\
& \oplus\left(\left[\left(1-p_{\epsilon(1)}\right) T_{1} 1_{B}\right] \otimes\left[\left(1-p_{\epsilon\left(k_{1}\right)}\right) S_{1} 1_{B}\right] \otimes \cdots \otimes\left[\left(1-p_{\epsilon\left(k_{m}\right)}\right) S_{m} 1_{B}\right]\right) .
\end{aligned}
$$

If $D_{1}$ is the $L R$-diagram obtained from $D_{0}$ by placing a node shaded $\epsilon(1)$ at the top and $D_{2}$ is the $L R$ diagram obtained from $D_{0}$ by placing a node $\epsilon(1)$ at the top and drawing a spine from this node to the top, then since

$$
L_{E\left(T_{1}\right)}\left(1-p_{\epsilon\left(k_{1}\right)}\right) S_{1} 1_{B}=\left(1-p_{\epsilon\left(k_{1}\right)}\right) L_{E\left(T_{1}\right)} S_{1} 1_{B},
$$

one easily sees that

$$
\begin{array}{r}
{\left[L_{E\left(T_{1}\right)}\left(1-p_{\epsilon\left(k_{1}\right)}\right) S_{1} 1_{B}\right] \otimes \cdots \otimes\left[\left(1-p_{\epsilon\left(k_{m}\right)}\right) S_{m} 1_{B}\right]=E_{D_{1}}\left(\mu_{1}\left(T_{1}\right), \mu_{2}\left(T_{2}\right), \ldots, \mu_{n}\left(T_{n}\right)\right)} \\
{\left[\left(1-p_{\epsilon(1)}\right) T_{1} 1_{B}\right] \otimes\left[\left(1-p_{\epsilon\left(k_{1}\right)}\right) S_{1} 1_{B}\right] \otimes \cdots \otimes\left[\left(1-p_{\epsilon\left(k_{m}\right)}\right) S_{m} 1_{B}\right]=E_{D_{2}}\left(\mu_{1}\left(T_{1}\right), \mu_{2}\left(T_{2}\right), \ldots, \mu_{n}\left(T_{n}\right)\right) .}
\end{array}
$$

Case 3: $m \neq 0$ and $\epsilon(1)=\epsilon\left(k_{1}\right)$. In this case, there is a spine in $D$ that reaches the top and is the same shading as $T_{1}$. Thus $\left(1-p_{\epsilon\left(k_{1}\right)}\right) S_{1} 1_{B} \in \stackrel{\circ}{\epsilon(1)}$, so

$$
\begin{aligned}
\lambda_{\epsilon(1)}\left(T_{1}\right) E_{D_{0}}\left(\mu_{2}\left(T_{2}\right), \ldots, \mu_{n}\left(T_{n}\right)\right)= & \left(\left[L_{p_{\epsilon(1)}\left(T_{1}\left(1-p_{\epsilon(1)}\right) S_{1} 1_{B}\right)}\left(1-p_{\epsilon\left(k_{2}\right)}\right) S_{2} 1_{B}\right] \otimes \cdots \otimes\left[\left(1-p_{\epsilon\left(k_{m}\right)}\right) S_{m} 1_{B}\right]\right) \\
& \oplus\left(\left[\left(1-p_{\epsilon(1)}\right) T_{1}\left(1-p_{\epsilon(1)}\right) S_{1} 1_{B}\right] \otimes \cdots \otimes\left[\left(1-p_{\epsilon\left(k_{m}\right)}\right) S_{m} 1_{B}\right]\right) .
\end{aligned}
$$


Expanding $T_{1}\left(1-p_{\epsilon(1)}\right) S_{1} 1_{B}=T_{1} S_{1} 1_{B}-T_{1} E\left(S_{1}\right)$, the above becomes

$$
\begin{aligned}
\lambda_{\epsilon(1)}\left(T_{1}\right) E_{D_{0}}\left(\mu_{2}\left(T_{2}\right), \ldots, \mu_{n}\left(T_{n}\right)\right)= & \left(\left[L_{E\left(T_{1} S_{1}\right)}\left(1-p_{\epsilon\left(k_{2}\right)}\right) S_{2} 1_{B}\right] \otimes \cdots \otimes\left[\left(1-p_{\epsilon\left(k_{m}\right)}\right) S_{m} 1_{B}\right]\right) \\
& \oplus(-1)\left(\left[L_{p_{\epsilon(1)}\left(T_{1} E\left(S_{1}\right)\right)}\left(1-p_{\epsilon\left(k_{2}\right)}\right) S_{2} 1_{B}\right] \otimes \cdots \otimes\left[\left(1-p_{\epsilon\left(k_{m}\right)}\right) S_{m} 1_{B}\right]\right) \\
& \oplus\left(\left[\left(1-p_{\epsilon(1)}\right) T_{1} S_{1} 1_{B}\right] \otimes \cdots \otimes\left[\left(1-p_{\epsilon\left(k_{m}\right)}\right) S_{m} 1_{B}\right]\right) \\
& \oplus(-1)\left(\left[\left(1-p_{\epsilon(1)}\right) T_{1} E\left(S_{1}\right)\right] \otimes \cdots \otimes\left[\left(1-p_{\epsilon\left(k_{m}\right)}\right) S_{m} 1_{B}\right]\right) .
\end{aligned}
$$

Let $D_{1}$ be the $L R$-diagram obtained from $D_{0}$ by placing a node shaded $\epsilon(1)$ at the top and terminating the left-most spine at this node, $D_{2}$ be the $L R$-diagram obtained by laterally refining $D_{1}$ by cutting the spine attached to the top node directly beneath the top node, $D_{3}$ be the $L R$-diagram obtained from $D_{0}$ by placing a node shaded $\epsilon(1)$ at the top and connecting this node to the left-most spine, and $D_{4}$ be the $L R$-diagram obtained by laterally refining $D_{3}$ by cutting the spine attached to the top node directly beneath the top node. As in the previous case, we see (by applying Lemma 5.2.1 if $m=1$ ) that

$$
\left[L_{E\left(T_{1} S_{1}\right)}\left(1-p_{\epsilon\left(k_{2}\right)}\right) S_{2} 1_{B}\right] \otimes \cdots \otimes\left[\left(1-p_{\epsilon\left(k_{m}\right)}\right) S_{m} 1_{B}\right]=E_{D_{1}}\left(\mu_{1}\left(T_{1}\right), \mu_{2}\left(T_{2}\right), \ldots, \mu_{n}\left(T_{n}\right)\right)
$$

and

$$
\left[\left(1-p_{\epsilon(1)}\right) T_{1} S_{1} 1_{B}\right] \otimes \cdots \otimes\left[\left(1-p_{\epsilon\left(k_{m}\right)}\right) S_{m} 1_{B}\right]=E_{D_{3}}\left(\mu_{1}\left(T_{1}\right), \mu_{2}\left(T_{2}\right), \ldots, \mu_{n}\left(T_{n}\right)\right) .
$$

We will demonstrate that

$$
\left[L_{p_{\epsilon(1)}\left(T_{1} E\left(S_{1}\right)\right)}\left(1-p_{\epsilon\left(k_{2}\right)}\right) S_{2} 1_{B}\right] \otimes \cdots \otimes\left[\left(1-p_{\epsilon\left(k_{m}\right)}\right) S_{m} 1_{B}\right]=E_{D_{2}}\left(\mu_{1}\left(T_{1}\right), \mu_{2}\left(T_{2}\right), \ldots, \mu_{n}\left(T_{n}\right)\right)
$$

and leave to the reader the proof that

$$
\left[\left(1-p_{\epsilon(1)}\right) T_{1} E\left(S_{1}\right)\right] \otimes \cdots \otimes\left[\left(1-p_{\epsilon\left(k_{m}\right)}\right) S_{m} 1_{B}\right]=E_{D_{4}}\left(\mu_{1}\left(T_{1}\right), \mu_{2}\left(T_{2}\right), \ldots, \mu_{n}\left(T_{n}\right)\right) .
$$

Notice that

$$
L_{p_{\epsilon(1)}\left(T_{1} E\left(S_{1}\right)\right)}=L_{p_{\epsilon(1)}\left(T_{1} R_{E\left(S_{1}\right)} 1_{B}\right)}=L_{p_{\epsilon(1)}\left(T_{1} 1_{B}\right) E\left(S_{1}\right)}=L_{p_{\epsilon(1)}\left(T_{1} 1_{B}\right)} L_{E\left(S_{1}\right)},
$$

and so

$$
L_{p_{\epsilon(1)}\left(T_{1} E\left(S_{1}\right)\right)}\left(1-p_{\epsilon\left(k_{2}\right)}\right) S_{2} 1_{B}=\left(1-p_{\epsilon\left(k_{2}\right)}\right) L_{p_{\epsilon(1)}\left(T_{1} 1_{B}\right)} L_{E\left(S_{1}\right)} S_{2} 1_{B}
$$

Thus, unless $m=1, L_{p_{\epsilon(1)}\left(T_{1} 1_{B}\right)}$ appears as it should in the definition of $E_{D_{2}}\left(\mu_{1}\left(T_{1}\right), \mu_{2}\left(T_{2}\right), \ldots, \mu_{n}\left(T_{n}\right)\right)$ although the $E\left(S_{1}\right)$ term may not be as it should. To obtain the desired result, we make the following corrections.

Recall that $S_{1}$ corresponds to the left-most-spine of $D_{0}$ reaching the top. Let $W \subseteq\{2, \ldots, n\}$ be the set of $k$ for which $T_{k}$ appears in the expression for $S_{1}$. Note that $S_{1}$ will be of the form $C_{E\left(S_{1}^{\prime}\right)} C_{E\left(S_{2}^{\prime}\right)} \cdots C_{E\left(S_{p-1}^{\prime}\right)} S_{p}^{\prime}$, where each $E\left(S_{k}^{\prime}\right)$ is the moment of a disjoint $\chi$-interval $W_{k}$ composed of blocks of $D_{2}$ with the property that $\min _{\prec_{\chi}}\left(W_{k}\right)$ and $\max _{\prec_{\chi}}\left(W_{k}\right)$ lie in the same block $(C$ denotes either $L$ or $R$, as appropriate). Observe that $W=\bigcup_{k=1}^{p} W_{k}$. Therefore, by Proposition 3.1.6

$$
E\left(S_{1}\right)=E\left(C_{E\left(S_{1}^{\prime}\right)} C_{E\left(S_{2}^{\prime}\right)} \cdots C_{E\left(S_{p-1}^{\prime}\right)} S_{p}^{\prime}\right)=E\left(S_{1}^{\prime \prime}\right) \cdots E\left(S_{p}^{\prime \prime}\right)
$$

where the $S_{1}^{\prime \prime}, \ldots, S_{p}^{\prime \prime}$ are $S_{1}^{\prime}, \ldots, S_{p}^{\prime}$ up to reordering by $\prec_{\chi}$. Let $W_{1}^{\prime}, \ldots, W_{k}^{\prime}$ be $W_{1}, \ldots, W_{k}$ under this new ordering.

Through Lemma 5.2.1 in the case $m=1$ and computations similar to the reverse of those used in cases 1 and 2 of Lemma 5.3.1 one can verify these terms can be moved into the correct positions.

Finally, it is clear that the coefficients of each $E_{D}\left(\mu_{1}\left(T_{1}\right), \ldots, \mu_{n}\left(T_{n}\right)\right)$ are correct for each $D \in L R^{\text {lat }}(\chi, \epsilon)$. Alternatively, one can check the coefficients in the second claim by noting that the coefficients did not depend on the algebra $B$, setting $B=\mathbb{C}$, and using [1, Corollary 4.2.5].

Theorem 7.1.4. Let $\left(\mathcal{A}, E_{\mathcal{A}}, \varepsilon\right)$ be a B-B-non-commutative probability space and let $\left\{\left(C_{k}, D_{k}\right)\right\}_{k \in K}$ be a family of pair of $B$-faces of $\mathcal{A}$. Then $\left\{\left(C_{k}, D_{k}\right)\right\}_{k \in K}$ are bi-free with amalgamation over $B$ if and only if for all $\chi:\{1, \ldots, n\} \rightarrow\{\ell, r\}, \epsilon:\{1, \ldots, n\} \rightarrow K$, and

$$
T_{k} \in\left\{\begin{array}{ll}
C_{\epsilon(k)} & \text { if } \chi(k)=\ell \\
D_{\epsilon(k)} & \text { if } \chi(k)=r
\end{array},\right.
$$


the formula

holds.

$$
E_{\mathcal{A}}\left(T_{1} \cdots T_{n}\right)=\sum_{\pi \in B N C(\chi)}\left[\sum_{\substack{\sigma \in B N C(\chi) \\ \pi \leq \sigma \leq \epsilon}} \mu_{B N C}(\pi, \sigma)\right] \mathcal{E}_{\pi}\left(T_{1}, \ldots, T_{n}\right)
$$

Proof. If $\left\{\left(C_{k}, D_{k}\right)\right\}_{k \in K}$ are bi-free with amalgamation over $B$, then Lemma 7.1 .3 implies the desired formula holds.

Conversely, suppose that the formula holds. By Theorem 3.2 .4 there exists a $B$ - $B$-bimodule with a specified $B$-vector state $(\mathcal{X}, \dot{\mathcal{X}}, p)$ and a unital homomorphism $\theta: \mathcal{A} \rightarrow \mathcal{L}(\mathcal{X})$ such that

$$
\theta\left(\varepsilon\left(b_{1} \otimes b_{2}\right)\right)=L_{b_{1}} R_{b_{2}}, \quad \theta\left(\mathcal{A}_{\ell}\right) \subseteq \mathcal{L}_{\ell}(\mathcal{X}), \quad \theta\left(\mathcal{A}_{r}\right) \subseteq \mathcal{L}_{r}(\mathcal{X}), \quad \text { and } \quad E_{\mathcal{L}(\mathcal{X})}(\theta(T))=E_{\mathcal{A}}(T)
$$

for all $b_{1}, b_{2} \in B$ and $T \in \mathcal{A}$. For each $k \in K$, let $\left(\mathcal{X}_{k}, \dot{\mathcal{X}}_{k}, p_{k}\right)$ be a copy of $(\mathcal{X}, \mathcal{X}, p)$ and $l_{k}$ and $r_{k}$ be copies of $\theta: \mathcal{A} \rightarrow \mathcal{L}\left(\mathcal{X}_{k}\right)$. Since the formula holds, Lemma 7.1.3 implies $\left\{\left(C_{k}, D_{k}\right)\right\}_{k \in K}$ are bi-free over $B$.

\section{Additivity of Operator-Valued Bi-Free Cumulants}

8.1. Equivalence of Bi-Freeness with Vanishing of Mixed Cumulants. We now state the operatorvalued analogue of the main result of [1, namely, that bi-freeness of families of pairs of $B$-faces is equivalent to the vanishing of their mixed cumulants.

Theorem 8.1.1. Let $(\mathcal{A}, E, \varepsilon)$ be a $B$-B-non-commutative probability space and let $\left\{\left(C_{k}, D_{k}\right)\right\}_{k \in K}$ be a family of pairs of $B$-faces from $\mathcal{A}$. Then $\left\{\left(C_{k}, D_{k}\right)\right\}_{k \in K}$ are bi-free with amalgamation over $B$ if and only if for all $\chi:\{1, \ldots, n\} \rightarrow\{\ell, r\}, \epsilon:\{1, \ldots, n\} \rightarrow K$, and

$$
T_{k} \in \begin{cases}C_{\epsilon(k)} & \text { if } \chi(k)=\ell \\ D_{\epsilon(k)} & \text { if } \chi(k)=r\end{cases}
$$

the equation

$$
\kappa_{1_{\chi}}\left(T_{1}, \ldots, T_{n}\right)=0
$$

holds unless $\epsilon$ is constant.

Proof. Suppose $\left\{\left(C_{k}, D_{k}\right)\right\}_{k \in K}$ are bi-free over $B$. Fix a shading $\epsilon:\{1, \ldots, n\} \rightarrow K$ and let $\chi:\{1, \ldots, n\} \rightarrow$ $\{\ell, r\}$. If $T_{1}, \ldots, T_{n}$ are operators as above, Theorem 7.1.4 implies

$$
\mathcal{E}_{1_{\chi}}\left(T_{1}, \ldots, T_{n}\right)=\sum_{\pi \in B N C(\alpha)}\left[\sum_{\substack{\sigma \in B N C(\chi) \\ \pi \leq \sigma \leq \epsilon}} \mu_{B N C}(\pi, \sigma)\right] \mathcal{E}_{\pi}\left(T_{1}, \ldots, T_{n}\right) .
$$

Therefore

$$
\mathcal{E}_{1_{\chi}}\left(T_{1}, \ldots, T_{n}\right)=\sum_{\substack{\sigma \in B N C(\chi) \\ \sigma \leq \epsilon}} \kappa_{\sigma}\left(T_{1}, \ldots, T_{n}\right)
$$

by Definition 6.1.3. Using the above formula, we will proceed inductively to show that $\kappa_{\sigma}\left(T_{1}, \ldots, T_{n}\right)=0$ if $\sigma \in B N C(\chi)$ and $\sigma \not \leq \epsilon$. The base case where $n=1$ is immediate.

For the inductive case, suppose the result holds for every $q<n$. Suppose $\epsilon$ is not constant and note $1_{\chi} \not \leq \epsilon$. Then

$$
\sum_{\sigma \in B N C(\chi)} \kappa_{\sigma}\left(T_{1}, \ldots, T_{n}\right)=\mathcal{E}_{1_{\chi}}\left(T_{1}, \ldots, T_{n}\right)=\sum_{\substack{\sigma \in B N C(\chi) \\ \sigma \leq \epsilon}} \kappa_{\sigma}\left(T_{1}, \ldots, T_{n}\right) .
$$

On the other hand, by induction and the recursive properties of bi-multiplicative functions, $\kappa_{\sigma}\left(T_{1}, \ldots, T_{n}\right)=$ 0 provided $\sigma \in B N C(\chi) \backslash\left\{1_{\chi}\right\}$ and $\sigma \not \leq \epsilon$. Consequently,

$$
\sum_{\sigma \in B N C(\chi)} \kappa_{\sigma}\left(T_{1}, \ldots, T_{n}\right)=\kappa_{1_{\chi}}\left(T_{1}, \ldots, T_{n}\right)+\sum_{\substack{\sigma \in B N C(\chi) \\ \sigma \leq \epsilon}} \kappa_{\sigma}\left(T_{1}, \ldots, T_{n}\right) .
$$

Combining these two equations gives $\kappa_{1_{\chi}}\left(T_{1}, \ldots, T_{n}\right)=0$, completing the inductive step. 
Conversely, suppose all mixed cumulants vanish. Then we have

$$
\begin{aligned}
\mathcal{E}_{1_{\chi}}\left(T_{1}, \ldots, T_{n}\right) & =\sum_{\sigma \in B N C(\chi)} \kappa_{\sigma}\left(T_{1}, \ldots, T_{n}\right) \\
& =\sum_{\substack{\sigma \in B N C(\chi) \\
\sigma \leq \epsilon}} \kappa_{\sigma}\left(T_{1}, \ldots, T_{n}\right) \\
& =\sum_{\substack{\sigma \in B N C(\chi) \\
\sigma \leq \epsilon}} \sum_{\substack{\pi \in B N C(\chi) \\
\pi \leq \sigma}} \mathcal{E}_{\pi}\left(T_{1}, \ldots, T_{n}\right) \mu_{B N C}(\pi, \sigma) \\
& =\sum_{\pi \in B N C(\chi)}\left[\sum_{\substack{\sigma \in B N C(\chi) \\
\pi \leq \sigma \leq \epsilon}} \mu_{B N C}(\pi, \sigma)\right] \mathcal{E}_{\pi}\left(T_{1}, \ldots, T_{n}\right) .
\end{aligned}
$$

Hence Theorem 7.1.4 implies $\left\{\left(C_{k}, D_{k}\right)\right\}_{k \in K}$ are bi-free over $B$.

8.2. Moment and Cumulant Series. In this section, we will begin the study of pairs of $B$-faces generated by operators.

Remark 8.2.1. Let $(\mathcal{A}, E, \varepsilon)$ be a $B$ - $B$-non-commutative probability space and let $(C, D)$ be a pair of $B$-faces such that

$$
\left.\left.C=\operatorname{alg}\left(\left\{L_{b} \mid b \in B\right\} \cup\left\{z_{i}\right\}_{i \in I}\right\}\right) \quad \text { and } \quad D=\operatorname{alg}\left(\left\{R_{b} \mid b \in B\right\} \cup\left\{z_{j}\right\}_{j \in J}\right\}\right) .
$$

We desire to compute the joint distribution of $(C, D)$ under $E$. Clearly it suffices to compute

$$
E\left(\left(C_{b_{1}} z_{\alpha(1)} C_{b_{1}^{\prime}}\right) \cdots\left(C_{b_{n}} z_{\alpha(n)} C_{b_{n}^{\prime}}\right)\right)=\mathcal{E}_{1_{\alpha}}\left(C_{b_{1}} z_{\alpha(1)} C_{b_{1}^{\prime}}, \ldots, C_{b_{n}} z_{\alpha(n)} C_{b_{n}^{\prime}}\right)
$$

where $\alpha:\{1, \ldots, n\} \rightarrow I \sqcup J, b_{1}, b_{1}^{\prime}, \ldots, b_{n}, b_{n}^{\prime} \in B$, and $C_{b_{k}}=L_{b_{k}}, C_{b_{k}^{\prime}}=L_{b_{k}^{\prime}}$ if $\alpha(k) \in I$ and $C_{b_{k}}=R_{b_{k}}$, $C_{b_{k}^{\prime}}=R_{b_{k}^{\prime}}$ if $\alpha(k) \in J$. However, because $\mathcal{E}$ is bi-multiplicative we can reduce this to computing

$$
\mathcal{E}_{1_{\chi_{\alpha}}}\left(z_{\alpha(1)} C_{b_{1}}, z_{\alpha(2)} C_{b_{2}}, \ldots, z_{\alpha(n-1)} C_{b_{n-1}}, z_{\alpha(n)}\right) .
$$

Similarly, to compute all possible cumulants, it suffices to compute

$$
\kappa_{1_{\chi \alpha}}\left(z_{\alpha(1)} C_{b_{1}}, z_{\alpha(2)} C_{b_{2}}, \ldots, z_{\alpha(n-1)} C_{b_{n-1}}, z_{\alpha(n)}\right) .
$$

As such we make the following definition.

Definition 8.2.2. Let $(\mathcal{A}, E, \varepsilon)$ be a $B$ - $B$-non-commutative probability space and let $(C, D)$ be a pair of $B$-faces such that

$$
\left.\left.C=\operatorname{alg}\left(\left\{L_{b} \mid b \in B\right\} \cup\left\{z_{i}\right\}_{i \in I}\right\}\right) \quad \text { and } \quad D=\operatorname{alg}\left(\left\{R_{b} \mid b \in B\right\} \cup\left\{z_{j}\right\}_{j \in J}\right\}\right) .
$$

The moment series of $z=\left(\left(z_{i}\right)_{i \in I},\left(z_{j}\right)_{j \in J}\right)$ is the collection of maps

$$
\left\{\mu_{\alpha}^{z}: B^{n-1} \rightarrow B \mid n \in \mathbb{N}, \alpha:\{1, \ldots, n\} \rightarrow I \sqcup J\right\}
$$

given by

$$
\mu_{\alpha}^{z}\left(b_{1}, \ldots, b_{n-1}\right)=\mathcal{E}_{1_{\chi_{\alpha}}}\left(z_{\alpha(1)} C_{b_{1}}, z_{\alpha(2)} C_{b_{2}}, \ldots, z_{\alpha(n-1)} C_{b_{n-1}}, z_{\alpha(n)}\right),
$$

where $C_{b_{k}}=L_{b_{k}}$ if $\alpha(k) \in I$ and $C_{b_{k}}=R_{b_{k}}$ otherwise.

Similarly, the cumulant series of $z$ is the collection of maps

$$
\left\{\kappa_{\alpha}^{z}: B^{n-1} \rightarrow B \mid n \in \mathbb{N}, \alpha:\{1, \ldots, n\} \rightarrow I \sqcup J\right\}
$$

given by

$$
\kappa_{\alpha}^{z}\left(b_{1}, \ldots, b_{n-1}\right)=\kappa_{1_{\chi \alpha}}\left(z_{\alpha(1)} C_{b_{1}}, z_{\alpha(2)} C_{b_{2}}, \ldots, z_{\alpha(n-1)} C_{b_{n-1}}, z_{\alpha(n)}\right) .
$$

Note that if $n=1$, we have $\mu_{\alpha}^{z}=E\left(z_{\alpha(1)}\right)=\kappa_{\alpha}^{z}$. 
Proposition 8.2.3. Let $(\mathcal{A}, E)$ be a $B$-B-non-commutative probability space, and for $\epsilon \in\left\{{ }^{\prime},{ }^{\prime \prime}\right\}$ let $\left\{z_{i}^{\epsilon}\right\}_{i \in I} \subset$ $\mathcal{A}_{\ell}$ and $\left\{z_{j}^{\epsilon}\right\}_{j \in J} \subset \mathcal{A}_{r}$. If

$$
C^{\epsilon}=\operatorname{alg}\left(\left\{L_{b}: b \in B\right\} \cup\left\{z_{i}^{\epsilon}\right\}_{i \in I}\right) \quad \text { and } \quad D^{\epsilon}=\operatorname{alg}\left(\left\{R_{b}: b \in B\right\} \cup\left\{z_{j}^{\epsilon}\right\}_{j \in J}\right)
$$

are such that $\left(C^{\prime}, D^{\prime}\right)$ and $\left(C^{\prime \prime}, D^{\prime \prime}\right)$ are bi-free, then

$$
\kappa_{\alpha}^{z^{\prime}+z^{\prime \prime}}=\kappa_{\alpha}^{z^{\prime}}+\kappa_{\alpha}^{z^{\prime \prime}} .
$$

Proof. This follows directly from Theorem 8.1.1.

\section{Multiplicative Convolution for Families of Pairs of $B$-Faces}

In this section, we will demonstrate how operator-valued bi-free cumulants involving products of operators may be computed. The main theorem of this section, Theorem 9.1.5, also gives rise to an extension of [1, Theorem 5.2.1] in the case $B=\mathbb{C}$.

\subsection{Operator-Valued Bi-Free Cumulants of Products.}

Notation 9.1.1. Given two partitions $\pi, \sigma \in B N C(\chi)$, we let $\pi \vee \sigma$ denote the smallest element of $B N C(\chi)$ greater than $\pi$ and $\sigma$.

Notation 9.1.2. Let $m, n \in \mathbb{N}$ with $m<n$ be fixed, and fix a sequence of integers

$$
k(0)=0<k(1)<\cdots<k(m)=n
$$

For $\chi:\{1, \ldots, m\} \rightarrow\{\ell, r\}$, we define $\widehat{\chi}:\{1, \ldots, n\} \rightarrow\{\ell, r\}$ via

$$
\widehat{\chi}(q)=\chi\left(p_{q}\right)
$$

where $p_{q}$ is the unique element of $\{1, \ldots, m\}$ such that $k\left(p_{q}-1\right)<q \leq k\left(p_{q}\right)$.

Remark 9.1.3. In the context of Notation 9.1.2, there exists an embedding of $B N C(\chi)$ into $B N C(\widehat{\chi})$ via $\pi \mapsto \widehat{\pi}$ where the $p^{\text {th }}$ node of $\pi$ is replaced by the block $(k(p-1)+1, \ldots, k(p))$. Observe that all of these nodes appear on the side $p$ was on originally. Alternatively, this map can be viewed as an analogue of the map on non-crossing partitions from [4, Notation 11.9] after applying $s_{\chi}^{-1}$.

It is easy to see that $\widehat{1_{\chi}}=1_{\widehat{\chi}}, \widehat{0_{\chi}}$ is the partition with blocks $\{(k(p-1)+1, \ldots, k(p))\}_{p=1}^{m}$, and $\pi \mapsto \widehat{\pi}$ is injective and preserves the partial ordering on $B N C$. Furthermore the image of $B N C(\chi)$ under this map is

$$
\widehat{B N C}(\chi)=\left[\widehat{0_{\chi}}, \widehat{1_{\chi}}\right]=\left[\widehat{0_{\chi}}, 1_{\widehat{\chi}}\right] \subseteq B N C(\widehat{\chi}) .
$$

Finally, since the lattice structure is preserved by this map, we see that $\mu_{B N C}(\sigma, \pi)=\mu_{B N C}(\widehat{\sigma}, \widehat{\pi})$.

Remark 9.1.4. Recall that since $\mu_{B N C}$ is the Möbius function on the lattice of bi-non-crossing partitions, we have for each $\sigma, \pi \in B N C(\chi)$ with $\sigma \leq \pi$ that

$$
\sum_{\substack{\tau \in B N C(\chi) \\
\sigma \leq \tau \leq \pi}} \mu_{B N C}(\tau, \pi)=\left\{\begin{array}{ll}
1 & \text { if } \sigma=\pi \\
0 & \text { otherwise }
\end{array} .\right.
$$

Therefore, it is easy to see that the partial Möbius inversion from [4, Proposition 10.11] holds in our setting; that is, if $f, g: B N C(\chi) \rightarrow B$ are such that

$$
f(\pi)=\sum_{\substack{\sigma \in B N C(\chi) \\ \sigma \leq \pi}} g(\sigma)
$$

for all $\pi \in B N C(\chi)$, then for all $\pi, \sigma \in B N C(\chi)$ with $\sigma \leq \pi$, we have the relation

$$
\sum_{\substack{\tau \in B N C(\chi) \\ \sigma \leq \tau \leq \pi}} f(\tau) \mu_{B N C}(\tau, \pi)=\sum_{\substack{\omega \in B N C(\chi) \\ \omega \vee \sigma=\pi}} g(\omega) .
$$

We now describe the operator-valued bi-free cumulants involving products of operators in terms of the above notation, following the spirit of [4, Theorem 11.12]. 
Theorem 9.1.5. Let $(\mathcal{A}, E, \varepsilon)$ be a $B$-B-non-commutative probability space, $m, n \in \mathbb{N}$ with $m<n$, $\chi$ : $\{1, \ldots, m\} \rightarrow\{\ell, r\}$, and

$$
k(0)=0<k(1)<\cdots<k(m)=n .
$$

If $\pi \in B N C(\chi)$ and $T_{k} \in \mathcal{A}_{\widehat{\chi}(k)}$ for all $k \in\{1, \ldots, n\}$, then

$$
\kappa_{\pi}\left(T_{1} \cdots T_{k(1)}, T_{k(1)+1} \cdots T_{k(2)}, \ldots, T_{k(m-1)+1} \cdots T_{k(m)}\right)=\sum_{\substack{\sigma \in B N C(\widehat{\chi}) \\ \sigma \vee \widehat{0} \chi}=\widehat{\pi}} \kappa_{\sigma}\left(T_{1}, \ldots, T_{n}\right) .
$$

In particular, for $\pi=1_{\chi}$, we have

$$
\kappa_{1_{\chi}}\left(T_{1} \cdots T_{k(1)}, T_{k(1)+1} \cdots T_{k(2)}, \ldots, T_{k(m-1)+1} \cdots T_{k(m)}\right)=\sum_{\substack{\sigma \in B N C(\widehat{\chi}) \\ \sigma \vee \widehat{0_{\chi}}=1_{\widehat{\chi}}}} \kappa_{\sigma}\left(T_{1}, \ldots, T_{n}\right) .
$$

Proof. For $j \in\{1, \ldots, m\}$, let $S_{j}=T_{k(j-1)+1} \cdots T_{k(j)}$. Then, by Definition 5.1.1,

$$
\begin{aligned}
\kappa_{\pi}\left(S_{1}, \ldots, S_{m}\right) & =\sum_{\substack{\tau \in B N C(\chi) \\
\tau \leq \pi}} \mathcal{E}_{\tau}\left(S_{1}, \ldots, S_{m}\right) \mu_{B N C}(\tau, \pi) \\
& =\sum_{\substack{\tau \in B N C(\chi) \\
\tau \leq \pi}} \mathcal{E}_{\widehat{\tau}}\left(T_{1}, \ldots, T_{n}\right) \mu_{B N C}(\widehat{\tau}, \widehat{\pi}) \\
& =\sum_{\substack{\sigma \in B N C(\widehat{\chi}) \\
\widehat{0_{\chi}} \leq \sigma \leq \widehat{\pi}}} \mathcal{E}_{\sigma}\left(T_{1}, \ldots, T_{n}\right) \mu_{B N C}(\sigma, \widehat{\pi}) \\
& =\sum_{\substack{\sigma \in B N C(\widehat{\chi}) \\
\sigma \vee \widehat{0_{\chi}}=\widehat{\pi}}} \kappa_{\sigma}\left(T_{1}, \ldots, T_{n}\right),
\end{aligned}
$$

with the last line following from Remark 9.1.4.

9.2. Multiplicative Convolution of Bi-Free Two-Faced Families. Recall from [1, Definition 5.1.1] that given any $\chi:\{1, \ldots, n\} \rightarrow\{\ell, r\}$ the (below on the left, above on the right) Kreweras complement of a bi-non-crossing partition $\pi \in B N C(\chi)$ is the element $K_{B N C}(\pi)$ of $B N C(\chi)$ obtained by applying $s_{\chi}$ to the (right) Kreweras complement in $N C(n)$ of $s_{\chi}^{-1} \cdot \pi$; that is,

$$
K_{B N C}(\pi):=s_{\chi} \cdot K_{N C}\left(s_{\chi}^{-1} \cdot \pi\right) .
$$

Using this Kreweras complement, [1, Theorem 5.2.1] constructed the multiplicative convolution of bi-free two-faced families where each pair of faces had a single left operator and a single right operator. Using Theorem 9.1.5, we can extend [1, Theorem 5.2.1] as follows, using the proof of [4, Theorem 14.4].

Proposition 9.2.1. Let $(\mathcal{A}, \varphi)$ be a non-commutative probability space. Let $z^{\prime}=\left(\left(z_{i}^{\prime}\right)_{i \in I},\left(z_{j}^{\prime}\right)_{j \in J}\right)$ and $z^{\prime \prime}=\left(\left(z_{i}^{\prime \prime}\right)_{i \in I},\left(z_{j}^{\prime \prime}\right)_{j \in J}\right)$ be bi-free two-faced families in $\mathcal{A}$, and set $z_{i}=z_{i}^{\prime} z_{i}^{\prime \prime}, z_{j}=z_{j}^{\prime \prime} z_{j}^{\prime}$ for $i \in I$ and $j \in J$. Then for $\alpha:\{1, \ldots, n\} \rightarrow I \sqcup J$, we have

$$
\kappa_{\chi}\left(z_{\alpha(1)}, \ldots, z_{\alpha(n)}\right)=\sum_{\pi \in B N C\left(\chi_{\alpha}\right)} \kappa_{\pi}\left(z_{\alpha(1)}^{\prime}, \ldots, z_{\alpha(n)}^{\prime}\right) \cdot \kappa_{K_{B N C}(\pi)}\left(z_{\alpha(1)}^{\prime \prime}, \ldots, z_{\alpha(n)}^{\prime \prime}\right) .
$$

Proof. Define $\widehat{\alpha}:\{1, \ldots, 2 n\} \rightarrow I \sqcup J$ by $\widehat{\alpha}(2 k-1)=\widehat{\alpha}(2 k)=\alpha(k)$ for $k \in\{1, \ldots, n\}$, and define $\epsilon$ : $\{1, \ldots, 2 n\} \rightarrow\left\{{ }^{\prime},{ }^{\prime \prime}\right\}$ by

$$
\epsilon(2 k-1)=\left\{\begin{array}{ll}
\prime & \text { if } \alpha(k) \in I \\
\prime \prime & \text { if } \alpha(k) \in J
\end{array} \quad \text { and } \quad \epsilon(2 k)= \begin{cases}\prime \prime & \text { if } \alpha(k) \in I \\
\prime & \text { if } \alpha(k) \in J\end{cases}\right.
$$

Using Theorem 9.1.5, we easily obtain

$$
\kappa_{\chi}\left(z_{\alpha(1)}, \ldots, z_{\alpha(n)}\right)=\sum_{\substack{\pi \in B N C\left(\chi_{\widehat{\alpha}}\right) \\ \pi \vee \sigma=1 \chi_{\widehat{\alpha}}}} \kappa_{\pi}\left(z_{\alpha(1)}^{\epsilon(1)}, z_{\alpha(1)}^{\epsilon(2)}, \ldots, z_{\alpha(n)}^{\epsilon(2 n-1)}, z_{\alpha(n)}^{\epsilon(2 n)}\right)
$$


where $\sigma=\left\{(1,2),(3,4), \ldots,\left(2 n-1,2_{n}\right)\right\}$. Since $z^{\prime}$ and $z^{\prime \prime}$ are bi-free, Theorem 8.1.1 (or simply 1, Theorem 4.3.1]) implies mixed bi-free cumulants vanish and thus only $\pi$ of the form $\pi=\pi^{\prime} \cup \pi^{\prime \prime}$ with $\pi^{\prime} \in$ $B N C\left(\left.\chi_{\widehat{\alpha}}\right|_{\left\{k \mid \epsilon(k)==^{\prime}\right\}}\right)$ and $\pi^{\prime \prime} \in B N C\left(\left.\chi_{\widehat{\alpha}}\right|_{\left\{k \mid \epsilon(k)==^{\prime \prime}\right\}}\right)$ will provide a non-zero contribution. However, for an arbitrary $\pi^{\prime} \in B N C\left(\left.\chi_{\widehat{\alpha}}\right|_{\left\{k \mid \epsilon(k)==^{\prime}\right\}}\right.$ ), it is elementary to see (for example, by the relations between the Kreweras complements for bi-non-crossing and non-crossing partitions) that there exists a unique $\pi^{\prime \prime} \in$ $B N C\left(\left.\chi_{\widehat{\alpha}}\right|_{\left\{k \mid \epsilon(k)==^{\prime \prime}\right\}}\right)$ such that $\left(\pi^{\prime} \cup \pi^{\prime \prime}\right) \vee \sigma=1_{\chi_{\widehat{\alpha}}} ;$ namely, $\pi^{\prime \prime}=K_{B N C}\left(\pi^{\prime}\right)$. Therefore, since we are in the scalar setting and $\kappa_{\tau}\left(T_{1}, \ldots, T_{n}\right)=\kappa_{\left.\tau\right|_{V}}\left(\left.\left(T_{1}, \ldots, T_{n}\right)\right|_{V}\right) \kappa_{\left.\tau\right|_{V^{c}}}\left(\left.\left(T_{1}, \ldots, T_{n}\right)\right|_{V^{c}}\right)$ whenever $\tau \in B N C\left(\chi^{\prime}\right)$ and $V$ is a block of $\pi$, we obtain the desired equation.

\section{Additional Examples of Bi-Free Families with Amalgamation}

In this section, we will demonstrate some useful techniques for constructing bi-free pairs of $B$-faces.

\subsection{Conjugation by Haar Bi-Unitaries.}

Definition 10.1.1. Consider the $B$-B-bimodule $\ell^{2}(\mathbb{Z}, B)=\left(B \delta_{0}\right) \oplus\left(B \delta_{0}\right)^{\perp}$. A concrete B-valued Haar bi-unitary is the invertible element

$$
U_{\text {Haar }} \in \mathcal{L}\left(\ell^{2}(\mathbb{Z}, B)\right)
$$

defined by

$$
U_{\text {Haar }}\left(\left(b_{k}\right)_{k \in K}\right)=\left(b_{k+1}\right)_{k \in K}
$$

for all $\left(b_{k}\right)_{k \in K} \in \ell^{2}(\mathbb{Z}, B)$. The moment series of

$$
z_{\text {Haar }}=\left(\left\{U_{\text {Haar }}, U_{\text {Haar }}^{-1}\right\},\left\{U_{\text {Haar }}, U_{\text {Haar }}^{-1}\right\}\right)
$$

will be called the B-valued Haar bi-unitary moment series.

Definition 10.1.2. Let $(\mathcal{A}, E, \varepsilon)$ be a $B$-B-non-commutative probability space. An abstract $B$-valued Haar bi-unitary in $\mathcal{A}$ is a pair of invertible elements $U_{\ell} \in \mathcal{A}_{\ell}$ and $U_{r} \in \mathcal{A}_{r}$ such that the moment series of $\left(\left\{\left(U_{\ell}, U_{\ell}^{-1}\right\},\left\{U_{r}, U_{r}^{-1}\right\}\right)\right.$ is equal to the $B$-valued Haar bi-unitary moment series. We will call

$$
\left(\operatorname{alg}\left(\varepsilon\left(B \otimes 1_{B}\right), U_{\ell}, U_{\ell}^{-1}\right), \operatorname{alg}\left(\varepsilon\left(1_{B} \otimes B^{\mathrm{op}}\right), U_{r}, U_{r}^{-1}\right)\right)
$$

the pair of $B$-faces generated by $\left(U_{\ell}, U_{r}\right)$.

Theorem 10.1.3. Let $(\mathcal{A}, E, \varepsilon)$ be a $B$-B-non-commutative probability space, let $(C, D)$ be a pair of $B$ faces in $(\mathcal{A}, E)$, and let $\left(U_{\ell}, U_{r}\right)$ be a B-valued Haar bi-unitary in $(\mathcal{A}, E, \varepsilon)$ such that $(C, D)$ is bi-free with amalgamation over $B$ from the pair of $B$-faces generated by $\left(U_{\ell}, U_{r}\right)$. Then the pairs of $B$-faces $(C, D)$ and $\left(U_{\ell}^{-1} C U_{\ell}, U_{r}^{-1} D U_{r}\right)$ are bi-free over $B$ and the joint distribution of $\left(U_{\ell}^{-1} C U_{\ell}, U_{r}^{-1} D U_{r}\right)$ is equal to the joint distribution of $(C, D)$.

Proof. Let $(\mathcal{X}, \mathcal{X}, \xi)$ be a $B$-bimodule that realizes $(C, D)$ with expectation $E$ (see Theorem 3.2.4). Since $(C, D)$ and the pair of $B$-faces generated by $\left(U_{\ell}, U_{r}\right)$ are bi-free over $B$, their joint distribution may be computed via the vacuum state with respect to their canonical actions on

$$
(\mathcal{X}, \mathcal{X}, \xi) *_{B}\left(\ell^{2}(\mathbb{Z}, B),\left(B \delta_{0}\right)^{\perp}, \delta_{0}\right),
$$

where both $U_{\ell}$ and $U_{r}$ act on $\ell^{2}(\mathbb{Z}, B)$ as $U_{\text {Haar }}$.

Let $\mathcal{Y}$ denote the $B$-submodule of $(\mathcal{X}, \mathcal{X}, \xi) *_{B}\left(\ell^{2}(\mathbb{Z}, B),\left(B \delta_{0}\right)^{\perp}, \delta_{0}\right)$ generated by all vectors of the form

$$
x_{0} \otimes\left(\delta_{-1} \otimes x_{1}^{\prime} \otimes \delta_{1}\right) \otimes x_{1} \otimes\left(\delta_{-1} \otimes x_{2}^{\prime} \otimes \delta_{1}\right) \otimes x_{2} \otimes \cdots \otimes\left(\delta_{-1} \otimes x_{n}^{\prime} \otimes \delta_{1}\right) \otimes x_{n}
$$

where $n \geq 0, x_{1}, \ldots, x_{n-1}, x_{1}^{\prime}, \ldots, x_{n}^{\prime} \in \dot{\mathcal{X}}$, and $x_{0}, x_{n} \in \mathcal{X}$. It is straightforward to verify that $\mathcal{Y}$ is invariant under the actions of both $(C, D)$ and $\left(U_{\ell}^{-1} C U_{\ell}, U_{r}^{-1} D U_{r}\right)$. As the vacuum vector is itself an element of $\mathcal{Y}$, the joint distribution of $(C, D)$ and $\left(U_{\ell}^{-1} C U_{\ell}, U_{r}^{-1} D U_{r}\right)$ may be computed purely by examining their actions on $\mathcal{Y}$.

To show that $(C, D)$ and $\left(U_{\ell}^{-1} C U_{\ell}, U_{r}^{-1} D U_{r}\right)$ are bi-free with amalgamation over $B$ and have the same joint distribution, we show that the joint distribution of $(C, D)$ and $\left(U_{\ell}^{-1} C U_{\ell}, U_{r}^{-1} D U_{r}\right)$ is the same as that of two bi-free copies of $(C, D)$ acting on a reduced free product space.

Let $\left(C_{1}, D_{1}\right)$ and $\left(C_{2}, D_{2}\right)$ be copies of $(C, D)$ acting on copies of $(\mathcal{X}, \dot{\mathcal{X}}, \xi)$ given by $\left(\mathcal{X}_{1}, \dot{\mathcal{X}}_{1}, \xi_{1}\right)$ and $\left(\mathcal{X}_{2}, \dot{\mathcal{X}}_{2}, \xi_{2}\right)$, respectively. Thus $\left(C_{1}, D_{1}\right) * *_{B}\left(C_{2}, D_{2}\right)$ has an induced action on $\left(\mathcal{X}_{1}, \dot{\mathcal{X}}_{1}, \xi_{1}\right) *_{B}\left(\mathcal{X}_{2}, \dot{\mathcal{X}}_{2}, \xi_{2}\right)$. 
Consider the map

$$
\Phi:\left(\mathcal{X}_{1}, \stackrel{\circ}{\mathcal{X}}_{1}, \xi_{1}\right) *_{B}\left(\mathcal{X}_{2}, \stackrel{\circ}{\mathcal{X}}_{2}, \xi_{2}\right) \rightarrow \mathcal{Y}
$$

defined as follows: for $n \geq 0, x_{1}, \ldots, x_{n-1} \in \stackrel{\circ}{\mathcal{X}}_{1}, x_{1}^{\prime}, \ldots, x_{n}^{\prime} \in \stackrel{\circ}{\mathcal{X}}_{2}$, and $x_{0}, x_{n} \in \mathcal{X}_{1}$, we define

$$
\Phi\left(x_{0} \otimes x_{1}^{\prime} \otimes x_{1} \otimes \cdots \otimes x_{n}^{\prime} \otimes x_{n}\right)=x_{0} \otimes\left(\delta_{-1} \otimes x_{1}^{\prime} \otimes \delta_{1}\right) \otimes x_{1} \otimes \cdots \otimes\left(\delta_{-1} \otimes x_{n}^{\prime} \otimes \delta_{1}\right) \otimes x_{n}
$$

It is elementary to verify that $\Phi$ is a $B$-bimodule isomorphism that sends the vacuum vector to the vacuum vector in $\mathcal{Y}$, that if $T \in C \cup D$ then viewing $T \in C_{1} \cup D_{1}$,

$$
\Phi(T \eta)=T \eta
$$

for all $\eta$, and that viewing $T \in C_{2} \cup D_{2}$,

$$
\Phi(T \eta)=U_{n}^{-1} T U_{n} \eta
$$

where $n=\ell$ if $T \in B_{2}$ and $n=r$ if $T \in C_{2}$. The result follows.

Remark 10.1.4. Given a two-faced family $(C, D)$ in a non-commutative probability space $(\mathcal{A}, \varphi)$, we may always enlarge $\mathcal{A}$ via the operator model from [1, Theorem 6.4.1] to include a $\mathbb{C}$-valued Haar bi-unitary $\left(U_{\ell}, U_{r}\right)$ bi-free from $(C, D)$.

10.2. Bi-Free Families where Left and Right Faces Commute. The following result demonstrates that if all of the left faces commute with all of the right faces, then, in certain circumstances, bi-freeness can be deduced from freeness of either the left faces or the right faces.

Theorem 10.2.1. Let $(\mathcal{A}, E, \varepsilon)$ be a B-B-non-commutative probability space acting on the B-bimodule $\mathcal{X}=B \oplus \dot{\mathcal{X}}$ (as in Theorem 3.2.4 for example) and let $\xi=1_{B} \oplus 0 \in \mathcal{X}$. Suppose $\left\{\left(C_{k}, D_{k}\right)\right\}_{k \in K}$ is a family of pairs of $B$-faces such that

(1) $C_{n}$ and $D_{m}$ commute for all $n, m \in K$, and

(2) for each $T \in D_{k}$ there exists an $S \in C_{k}$ such that $T \xi=S \xi$.

Then $\left\{\left(C_{k}, D_{k}\right)\right\}_{k \in K}$ are bi-free with amalgamation over $B$ if and only if $\left\{C_{k}\right\}_{k \in K}$ are free with amalgamation over $B$. Therefore, if $\left\{C_{k}\right\}_{k \in K}$ are free with amalgamation over $B$ then $\left\{D_{k}\right\}_{k \in K}$ are free with amalgamation over $B$.

Proof. If $\left\{\left(C_{k}, D_{k}\right)\right\}_{k \in K}$ are bi-free over $B$ then it is clear that $\left\{C_{k}\right\}_{k \in K}$ are free over $B$ and $\left\{D_{k}\right\}_{k \in K}$ are free over $B$.

Suppose $\left\{C_{k}\right\}_{k \in K}$ are free over $B$. To show that $\left\{\left(C_{k}, D_{k}\right)\right\}_{k \in K}$ are bi-free over $B$ we need to verify the operator polynomials

$$
E\left(T_{1} \cdots T_{n}\right)=\sum_{\pi \in B N C(\chi)}\left[\sum_{\substack{\sigma \in B N C(\chi) \\ \pi \leq \sigma \leq \epsilon}} \mu_{B N C}(\pi, \sigma)\right] E_{\pi}\left(T_{1}, \ldots, T_{n}\right)
$$

whenever $\chi:\{1, \ldots, n\} \rightarrow\{\ell, r\}, \epsilon:\{1, \ldots, n\} \rightarrow K$, and $T_{k} \in C_{\epsilon(k)}$ if $\chi(k)=\ell$, and $T_{k} \in D_{\epsilon(k)}$ if $\chi(k)=r$. Note if $\chi^{-1}(\{\ell\})=\{1, \ldots, n\}$, the freeness of $\left\{C_{k}\right\}_{k \in K}$ implies that these polynomials hold. Thus we will proceed by induction on $\left|\chi^{-1}(\{r\})\right|$ with the base case of $\left|\chi^{-1}(\{r\})\right|=0$ complete.

Let $s$ be the permutation such that

$$
\chi^{-1}(\{\ell\})=\{s(1)<\ldots<s(k)\} \quad \text { and } \quad \chi^{-1}(\{r\})=\{s(k+1)<\ldots<s(n)\} .
$$

Let $\hat{\epsilon}=\epsilon \circ s$, and $\hat{\chi}=\chi \circ s$. Note that replacing $\chi$ by $\hat{\chi}$ and $\epsilon$ by $\hat{\epsilon}$ corresponds to moving the right nodes beneath the left ones, without changing their relative order. In particular, due to commutation, $T_{s(1)} \cdots T_{s(n)}=T_{1} \cdots T_{n}$ and bi-multiplicativity implies

$$
E\left(T_{1} \cdots T_{n}\right)=\sum_{\pi \in B N C(\chi)}\left[\sum_{\substack{\sigma \in B N C(\chi) \\ \pi \leq \sigma \leq \epsilon}} \mu_{B N C}(\pi, \sigma)\right] E_{\pi}\left(T_{1}, \ldots, T_{n}\right)
$$


if and only if

$$
E\left(T_{s(1)} \cdots T_{s(n)}\right)=\sum_{\pi \in B N C(\hat{\chi})}\left[\sum_{\substack{\sigma \in B N C(\hat{\chi}) \\ \pi \leq \sigma \leq \hat{\epsilon}}} \mu_{B N C}(\pi, \sigma)\right] E_{\pi}\left(T_{s(1)}, \ldots, T_{s(n)}\right) .
$$

To reduce the number of right operators, we note that $\hat{\chi}(n)=r$ and select $S \in C_{\hat{\epsilon}(n)}$ such that $S \xi=T_{s(n)} \xi$. Define $\bar{\chi}:\{1, \ldots, n\} \rightarrow\{\ell, r\}$ by

$$
\bar{\chi}(p)=\left\{\begin{array}{ll}
\hat{\chi}(p) & \text { if } p<n \\
\ell & \text { if } p=n
\end{array} .\right.
$$

Clearly there is a canonical map from $B N C(\hat{\chi})$ to $B N C(\bar{\chi})$ that takes $\pi \in B N C(\hat{\chi})$ and produces $\bar{\pi} \in$ $B N C(\bar{\chi})$ by moving the bottom node of $\pi$ from a right node to a left node while keeping all strings attached. Note that

$$
\sum_{\substack{\sigma \in B N C(\hat{\chi}) \\ \pi \leq \sigma \leq \hat{\epsilon}}} \mu_{B N C}(\pi, \sigma)=\sum_{\substack{\sigma \in B N C(\bar{\chi}) \\ \bar{\pi} \leq \bar{\sigma} \leq \hat{\epsilon}}} \mu_{B N C}(\bar{\pi}, \bar{\sigma}) .
$$

Moreover, $S \xi=T_{s(n)} \xi$ implies that for all $\pi \in B N C(\hat{\chi})$,

$$
E_{\pi}\left(T_{s(1)}, \ldots, T_{s(n)}\right)=E_{\bar{\pi}}\left(T_{s(1)}, \ldots, T_{s(n-1)}, S\right)
$$

Then we see that

$$
E\left(T_{s(1)} \cdots T_{s(n)}\right)=\sum_{\pi \in B N C(\hat{\chi})}\left[\sum_{\substack{\sigma \in B N C(\hat{\chi}) \\ \pi \leq \sigma \leq(\hat{\epsilon})}} \mu_{B N C}(\pi, \sigma)\right] E_{\pi}\left(T_{s(1)}, \ldots, T_{s(n)}\right) .
$$

if and only if

$$
E\left(T_{s(1)} \cdots T_{s(n-1)} S\right)=\sum_{\bar{\pi} \in B N C(\bar{\chi})}\left[\sum_{\substack{\sigma \in B N C(\bar{\chi}) \\ \bar{\pi} \leq \bar{\sigma} \leq \hat{\epsilon}}} \mu_{B N C}(\bar{\pi}, \bar{\sigma})\right] E_{\bar{\pi}}\left(T_{s(1)}, \ldots, T_{s(n-1)}, S\right) .
$$

Since the last equation holds by our inductive hypothesis, the proof is complete.

Corollary 10.2.2. Let $(\mathcal{A}, E, \varepsilon)$ be a B-B-non-commutative probability space acting on the B-bimodule $\mathcal{X}=B \oplus \dot{\mathcal{X}}$ and let $\xi=1_{B} \oplus 0 \in \mathcal{X}$. Suppose $\left\{\left(C_{k}, D_{k}\right)\right\}_{k \in K}$ is a family of pairs of B-faces such that

(1) $C_{n}$ and $D_{m}$ commute for all $n, m \in K$, and

(2) $\left\{T \xi: T \in C_{k}\right\}=\left\{S \xi: S \in D_{k}\right\}$ for all $k \in K$.

Then $\left\{\left(C_{k}, D_{k}\right)\right\}_{k \in K}$ are bi-free over $B$ if and only if $\left\{C_{k}\right\}_{k \in K}$ are free over $B$ if and only if $\left\{D_{k}\right\}_{k \in K}$ are free over $B$.

Corollary 10.2.3. Let $(\mathcal{A}, \varphi)$ be a non-commutative probability space acting on a pointed vector space $(X, \xi)$ with $\xi$ realizing $\varphi$. Suppose $\left\{\left(C_{k}, D_{k}\right)\right\}_{k \in K}$ is a family of pairs of faces such that

(1) $C_{n}$ and $D_{m}$ commute for all $n, m \in K$, and

(2) for each $T \in D_{k}$ there exists an $S \in C_{k}$ such that $T \xi=S \xi$.

Then $\left\{\left(C_{k}, D_{k}\right)\right\}_{k \in K}$ are bi-free if and only if $\left\{C_{k}\right\}_{k \in K}$ are free. Therefore, if $\left\{C_{k}\right\}_{k \in K}$ are free then $\left\{D_{k}\right\}_{k \in K}$ are free.

The above is particularly interesting as it enables the transference of freeness from one algebra to another.

\section{REFERENCES}

[1] I. Charlesworth, B. Nelson, and P. Skoufranis, On Two-Faced Families of Non-Commutative Random Variables (2014), 26 pp., available at arXiv:1403.4907

[2] M. Mastank and A. Nica, Double-ended queues and joint moments of left-right canonical operators on full Fock space (2013), 28 pp., available at arXiv:1312.0269.

[3] A. Nica, D. Shylakhtenko, and R. Speicher, Operator-valued distributions: I. Characterizations of freeness, Int. Math. Res. Notices 29 (2002), 1509-1538. 
[4] A. Nica and R. Speicher, Lectures on the Combinatorics of Free Probability, London Mathematics Society Lecture Notes Series, vol. 335, Cambridge University Press, 2006.

[5] R. Speicher, Combinatorial theory of the free product with amalgamation and operator-valued free probability theory, Memoirs of the American Mathematical Society, vol. 627, American Mathematical Soc., 1998.

[6] D. V. Voiculescu, Operations on certain non-commutative operator-valued random variables, Astérisque 232 (1995), $243-$ 375 .

[7] _ Free Probability for Pairs of Faces I (2013), 33 pp., available at arXiv:1306.6082

Department of Mathematics, UCla, Los Angeles, California, 90095, USA

E-mail address: ilc@math.ucla.edu, bnelson6@math.ucla.edu, pskoufra@math.ucla.edu 